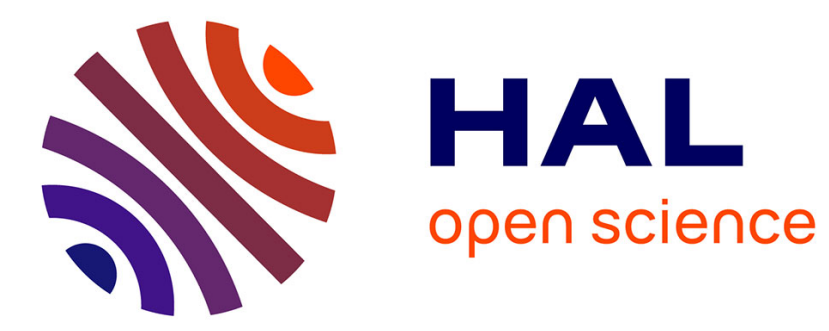

\title{
Bifurcation diagrams and heteroclinic networks of octagonal H-planforms
}

\author{
Grégory Faye, Pascal Chossat
}

\section{To cite this version:}

Grégory Faye, Pascal Chossat. Bifurcation diagrams and heteroclinic networks of octagonal Hplanforms. Journal of Nonlinear Science, 2012, 22 (1), pp.277-325. 10.1007/s00332-011-9118-x . hal-00807364

\section{HAL Id: hal-00807364 \\ https://hal.science/hal-00807364}

Submitted on 3 Apr 2013

HAL is a multi-disciplinary open access archive for the deposit and dissemination of scientific research documents, whether they are published or not. The documents may come from teaching and research institutions in France or abroad, or from public or private research centers.
L'archive ouverte pluridisciplinaire HAL, est destinée au dépôt et à la diffusion de documents scientifiques de niveau recherche, publiés ou non, émanant des établissements d'enseignement et de recherche français ou étrangers, des laboratoires publics ou privés. 


\title{
Bifurcation diagrams and heteroclinic networks of octagonal H-planforms
}

\author{
Grégory Faye ${ }^{1}$ and Pascal Chossat ${ }^{1,2}$ \\ ${ }^{1}$ NeuroMathComp Laboratory, INRIA, Sophia Antipolis, ENS Paris, France \\ ${ }^{2}$ J-A Dieudonné Laboratory, CNRS and University of Nice Sophia-Antipolis, Parc Valrose, 06108 Nice Cedex \\ 02, France
}

October 11, 2011

\begin{abstract}
This paper completes the classification of bifurcation diagrams for H-planforms in the Poincaré disc $\mathcal{D}$ whose fundamental domain is a regular octagon. An H-planform is a steady solution of a PDE or integro-differential equation in $\mathcal{D}$, which is invariant under the action of a lattice subgroup $\Gamma$ of $U(1,1)$, the group of isometries of $\mathcal{D}$. In our case $\Gamma$ generates a tiling of $\mathcal{D}$ with regular octagons. This problem was introduced as an example of spontaneous pattern formation in a model of image feature detection by the visual cortex where the features are assumed to be represented in the space of structure tensors. Under "generic" assumptions the bifurcation problem reduces to an ODE which is invariant by an irreducible representation of the group of automorphisms $\mathcal{G}$ of the compact Riemann surface $\mathcal{D} / \Gamma$. The irreducible representations of $\mathcal{G}$ have dimension one, two, three and four. The bifurcation diagrams for the representations of dimension less than four have already been described and correspond to already well known goup actions. In the present work we compute the bifurcation diagrams for the remaining three irreducible representations of dimension four, thus completing the classification. In one of these cases, there is generic bifurcation of a heteroclinic network connecting equilibria with two different orbit types.
\end{abstract}

Keywords: Equivariant bifurcation analysis; neural fields; Poincaré disc; heteroclinic network.

\section{Introduction}

Pattern formation through Turing mechanism is a well-known phenomenon [28]. For a system of reactiondiffusion equations defined in $\mathbb{R}^{p}$, with $p=2$ or 3 say, it occurs when a neutrally stable linear mode is selected as a basic, homogeneous state, when a bifurcation parameter reaches a critical value. For the analysis of this phenomenon, the assumption that the system is invariant under Euclidean transformations in the plane is essential. Any Fourier mode whose wave vector has critical length is a neutral stable mode and a consequence of the rotational symmetry of the system is that the kernel of linearized problem at the bifurcation point is infinite dimensional. However in experiments as well as in numerical simulations it is often seen that the patterns which emerge above threshold, although they have a wave number equal or close to the critical one, are associated with a finite (and small) number of wave vectors which generate a spatially periodic pattern in the plane. This pattern is invariant under the action of 
a discrete translation subgroup $\Gamma$ of $\mathbb{R}^{p}$. By looking at the class of $\Gamma$-periodic states, or by looking at the system projected onto the torus $\mathbb{R}^{p} / \Gamma$, one renders the spectrum of the linearized problem discrete: the critical wave vectors are finite in number, hence the critical eigenvalue has finite multiplicity and standard methods of equivariant bifurcation theory (see [11, 23]) can be applied to compute bifurcated solutions within the class of $\Gamma$-periodic states. Such solutions are called "planforms".

In a recent paper [14], a similar problem arose, but instead of being posed in the Euclidean plane, it was posed on the hyperbolic plane or, more conveniently, on the Poincaré disc. The problem originates from modeling the cortical perception of visual features, which we now briefly describe.

Neuronal representations of the external world are often based on the selectivity of the responses of individual neurons to external features. It has been well documented that neurons in the primary visual cortex respond preferentially to visual stimuli that have specific features such as orientation, spatial frequency, etc. Subgroups of inhibitory and excitatory neurons tuned to a particular feature of an external stimulus form what is called a Hubel and Wiesel hypercolumn of the visual area V1 in the cortex, in roughly $1 \mathrm{~mm}^{2}$ of cortical surface. Modeling the processing of image orientations has led Wilson and Cowan $[43,44]$ to derive a nonlinear integro-differential description of the evolution of the average action potential $V$ in the hypercolumns. In an attempt to extend this model to other features (edge and corner detection, contrast...), it was proposed by [13] to assume that hypercolumns are sensitive to a nonlinear representation of the image's first order derivatives called the structure tensor [6, 32]. Hence the average action potential is now a function of the structure tensor and time. Structure tensors are essentially $2 \times 2$ symmetric, positive definite matrices. They therefore live in a solid open cone in $\mathbb{R}^{3}$, which is a Riemannian manifold foliated by hyperbolic planes. By a suitable change of coordinates, the hyperbolic plane can be further identified with the Poincaré disc $\mathcal{D}=\{z \in \mathbb{C}|| z \mid<1\}$. There is therefore an isomorphism between the space of structure tensors and the product space $\mathbb{R}_{*}^{+} \times \mathcal{D}$, the distance on which being given by

$$
d\left(\delta, z ; \delta^{\prime}, z^{\prime}\right)=\sqrt{\log ^{2}\left(\frac{\delta}{\delta^{\prime}}\right)+\operatorname{arctanh} \frac{\left|z-z^{\prime}\right|^{2}}{\left|1-\bar{z} z^{\prime}\right|}}
$$

where the second term under the radical is the usual "hyperbolic" distance in $\mathcal{D}$.

The Wilson-Cowan equation on the space of structure tensors has the form

$$
\partial_{t} V(\delta, z, t)=-V(\delta, z, t)+\int_{\mathbb{R}_{*}^{+} \times \mathcal{D}} W\left(\delta, z, \delta^{\prime}, z^{\prime}\right) S\left(\mu V\left(\delta^{\prime}, z^{\prime}, t\right)\right) \operatorname{dm}\left(\delta^{\prime}, z^{\prime}\right)+I_{\text {ext }}(\delta, z, t)
$$

The nonlinearity $S$ is a smooth sigmoidal function $(S(x) \rightarrow \pm 1$ as $x \rightarrow \pm \infty)$ with $S(0)=0$. Note that $V=0$ is always solution (trivial state) when $I_{\text {ext }}=0$. The parameter $\mu$ describes the stiffness 
of the sigmoid. In the function $I_{e x t}$ we englobe the information coming from the lateral geniculate body (directly linked to the image seen in the visual field), but also feedbacks coming from other visual areas such as $M T, V 2$ and $V 4$. The coupling function $W\left(\delta, z, \delta^{\prime}, z^{\prime}\right)$ expresses interactions between populations of neurons of types $(\delta, z)$ and $\left(\delta^{\prime}, z^{\prime}\right)$ in the hypercolumn. It is natural to assume that the connectivity function $W$ does in fact only depend upon the distance between $(\delta, z)$ and $\left(\delta^{\prime}, z^{\prime}\right)$ : $W\left(\delta, z, \delta, z^{\prime}\right)=w\left(d\left(W\left(\delta, z ; \delta, z^{\prime}\right)\right)\right.$. Under this hypothesis, $W$ is invariant by any isometric transformation in the space of structure tensors. If in addition $I_{e x t}=0$ (no input), Equation (2) itself is equivariant by any isometric transformation. Thus $U(1,1)$ is the symmetry group of (2) when $I_{\text {ext }}=0$.

We shall assume from now on that $W$ has the above invariant form and that $I_{\text {ext }}=0$. We look at possible bifurcations from the fully symmetric state $V=0$ as the stifness parameter $\mu$ is varied. Remark that for all $\mu$, the symmetric state $V=0$ is a solution of equation (2) and its uniqueness has been discussed in [18]. As was shown in [14], we are able to neglect the dependence on $\delta \in \mathbb{R}_{*}^{+}$as it does not play a significant role in the analysis that follows. Therefore Equation (2) is posed on the 2D hyperbolic surface $\mathcal{D}$ from now on.

Spectral analysis in $\mathcal{D}$ requires the tools introduced by Helgason to perform Fourier analysis with respect to the coordinates in the Poincaré disc, namely the expansion of the solutions of the linearized system in elementary eigenfunctions of the Laplace-Beltrami operator [27]. These elementary eigenfunctions $e_{\rho, b}(z)$ depend on the "wave number" $\rho \in \mathbb{R}$ and point $b$ on the boundary of the unit disc, and correspond to eigenvalues $1 / 4+\rho^{2}$. Note that the eigenvalues are independant of the angle $b$. The function $e_{\rho, b}$ corresponds to a wavy pattern in $\mathcal{D}$ which is invariant along horocycles ${ }^{1}$ with base point $b$ and of wave type along the geodesics with direction $b$. These functions are the hyperbolic counterparts of the elementary wavy eigenfunctions of the Laplacian in $\mathbb{R}^{2}$. As shown in [13], a critical value $\mu_{c}$ exists, but the same kind of rotational degeneracy occurs in this bifurcation problem as in Euclidean space: if $\rho_{c}$ is the critical wave number, any $e_{\rho_{c}, b}$ is a neutrally stable eigenfunction, independently of the value of $b$. Moreover the spectrum is continuous. In order to apply equivariant bifurcation theory, we therefore need to look at a class of solutions which are periodic in $\mathcal{D}$, that is, solutions which are invariant under the action of a discrete subgroup $\Gamma$ of $U(1,1)$ whose fundamental domain is a polygon. Such a subgroup is called a cocompact Fuchsian group and we can restrict further to look for such groups which contain no elliptic elements nor reflections ${ }^{2}$. Tilings of the Poincaré disc have very different properties from tilings of the Euclidean plane. In particular tilings exist with polygons having an arbitrary number of sides, while in $\mathbb{R}^{2}$ only rectangular, square and hexagonal periodic tilings exist. Now the problem comes back to looking for bifurcated solutions of the equation defined on the quotient space $\mathcal{D} / \Gamma$, which is a compact

\footnotetext{
${ }^{1}$ A horocycle with base point $b$ is a circle in $\mathcal{D}$, tangent at $b$ to $\partial \mathcal{D}$.

${ }^{2}$ These subgroups of $S U(1,1)$ contain only hyperbolic elements and are the exact counterparts of discrete translation subgroups of $\mathbb{R}^{p}$. They are called "torsion-free" cocompact Fuchsian groups, see [29].
} 
Riemann surface; i.e. the spectrum of the linearized operator is discrete and consists of eigenvalues with finite multiplicity.

This approach to the problem was presented in [14] and an example was studied, namely the case where the group $\Gamma$ corresponds to a tiling of $\mathcal{D}$ with regular octagons. This particular choice was initially motivated and explained in [13] where families of subgroups of $U(1,1)$ were identified to naturally arise from the retinal input to the hypercolumns in the visual area V1 such as the group $\Gamma$. In this case $\Gamma$ is generated by four hyperbolic transformations which are rotated from each other by angles $k \pi / 4$ $(k=1,2,3)$, and $\mathcal{D} / \Gamma$ is a double torus (genus 2 surface). Moreover the group of automorphisms $\mathcal{G}$ of $\mathcal{D} / \Gamma$ is known and has 96 elements. Restricting to the class of $\Gamma$-periodic functions, our bifurcation problem is now reduced to an equation that is invariant under the action of $\mathcal{G}$. By standard center manifold reduction, this equation can be projected onto the critical eigenspace of the linearized operator. In our case the critical eigenvalue is 0 ("steady state" bifurcation) and its eigenspace is an absolutely irreducible representation space of the group $\mathcal{G}$.

In the same paper we listed and described the 13 irreducible representations of $\mathcal{G}$ which we name $\chi_{1}, \cdots, \chi_{13}$ : representations $\chi_{1}, \cdots, \chi_{4}$ have dimension one, $\chi_{5}, \chi_{6}$ have dimension two, $\chi_{7}, \cdots, \chi_{10}$ have dimension three and $\chi_{11}, \cdots, \chi_{13}$ have dimension four. Each of these cases leads to a different bifurcation diagram. We listed all maximal isotropy subgroups and showed that their fixed point subspaces are one dimensional, hence we found all branches of equilibria with maximal isotropy types by applying the Equivariant Branching Lemma [23]. Moreover it was shown that the bifurcation problems for the 2D cases is equivalent to problems with triangular symmetry, and in the $3 \mathrm{D}$ cases, to problems with octahedral symmetry. It follows that the bifurcation diagrams in these cases are known and show generically no other bounded dynamics than the trivial ones associated with the equilibria with maximal isotropy. There is no such identification in the $4 \mathrm{D}$ case. The aim of this paper is to fill this gap, by studying the bifurcation diagrams and local dynamics in the $4 \mathrm{D}$ irreducible representation spaces of $\mathcal{G}$. This will require a precise knowledge of these representations and of the Taylor expansion of vector fields which are equivariant by these representations (up to a sufficient order).

The structure of the paper is as follows:

- In section 2 we introduce the octagonal lattice and its symmetries, we recall the structure of the group $\mathcal{G}$ of automorphisms of $\mathcal{D} / \Gamma$ and its irreducible representations. We also recall the main result of [14] about the bifurcation of H-planforms in this case.

- In section 3, we study the case of the 4-dimensional irreducible representations $\chi_{12}, \chi_{13}$ and show that the system is locally "gradient-like", implying that the only $\omega$-limit sets are equilibria. The main results of this section are stated in theorems 2 and 3. We include this case for completeness 
although it does not show more complex phenomena than bifurcation of equilibria with maximal isotropy. Computations are sketched.

- In section 4 , we focus on the analysis of the 4-dimensional irreducible representations $\chi_{11}$. The computation of quintic equivariant vector fields is necessary to get a complete bifurcation diagram in the fixed point planes. We show that for some open range of the coefficients of quintic order terms, bifurcation with submaximal isotropy does occur. The main results are presented in theorems 4 and 5 .

- In section 5, we both show the existence of a heteroclinic network and adress the question of its asymptotic stability, which we illustrate with numerical simulations.

\section{Basic facts and results}

In this section we recall some basic facts about the Poincaré disc and its isometries and we summarize results of [14] which will be useful in subsequent analysis.

\subsection{The regular octagonal lattice and its symmetries}

We recall that the direct (orientation preserving) isometries of the Poincaré disc $\mathcal{D}$ form the group $\mathrm{SU}(1,1)$ of $2 \times 2$ Hermitian matrices with determinant equal to 1 . Given

$$
\gamma=\left(\begin{array}{cc}
\alpha & \beta \\
\bar{\beta} & \bar{\alpha}
\end{array}\right) \text { such that }|\alpha|^{2}-|\beta|^{2}=1,
$$

the corresponding isometry in $\mathcal{D}$ is defined by:

$$
\gamma \cdot z=\frac{\alpha z+\beta}{\bar{\beta} z+\bar{\alpha}}, \quad z \in \mathcal{D}
$$

Orientation reversing isometries of $\mathcal{D}$ are obtained by composing any transformation (3) with the reflection $\kappa: z \rightarrow \bar{z}$. The full symmetry group of the Poincaré disc is therefore:

$$
\mathrm{U}(1,1)=\mathrm{SU}(1,1) \cup \kappa \cdot \mathrm{SU}(1,1)
$$

Transformations in $\mathrm{SU}(1,1)$ can be of three types: elliptic (those belong to the conjugacy class of usual rotations centered at the origin of the disc), parabolic (those have a unique fixed point which lies on the boundary of $\mathcal{D}$ ) and hyperbolic (two fixed points on $\partial \mathcal{D}$ ). 


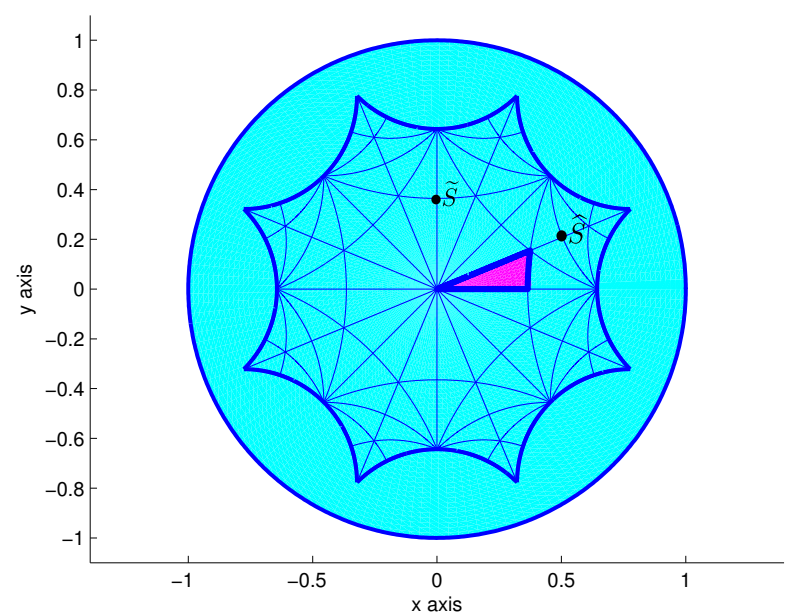

Figure 1: Tesselation of the hyperbolic octagon $\mathcal{O}$ with congruent triangles.

The octagonal lattice group $\Gamma$ is generated by the following four hyperbolic transformations (boosts), see $[5]$ :

$$
g_{0}=\left(\begin{array}{cc}
1+\sqrt{2} & \sqrt{2+2 \sqrt{2}} \\
\sqrt{2+2 \sqrt{2}} & 1+\sqrt{2}
\end{array}\right)
$$

and $g_{j}=r_{j \pi / 4} g_{0} r_{-j \pi / 4}, j=1,2,3$, where $r_{\varphi}$ indicates the rotation of angle $\varphi$ around the origin in $\mathcal{D}$. The fundamental domain of the lattice is a regular octagon $\mathcal{O}$ as shown in Figure 1. The opposite sides of the octagon are identified by periodicity, so that the corresponding quotient surface $\mathcal{D} / \Gamma$ is isomorphic to a "double doughnut" (genus two surface) [5]. The fundamental octagon $\mathcal{O}$ can be further decomposed into 96 congruent triangles (see Figure 1 ) with angles $\pi / 2, \pi / 3$ and $\pi / 8$. By applying reflections through the sides of one triangle (like the purple one in Figure 1) and iterating the process, applying if necessary a translation in $\Gamma$ to get the resulting triangle back to $\mathcal{O}$, one fills out the octagon. The set of all these transformations $(\bmod \Gamma)$ is isomorphic to the group of automorphisms of $\mathcal{D} / \Gamma$, we call it $\mathcal{G}$. Let us call $P, Q, R$ the vertices of the red triangle in Figure 1 which have angles $\pi / 8, \pi / 2$ and $\pi / 3$ respectively.

Definition 1. We set :

(i) $\kappa, \kappa^{\prime}$ and $\kappa^{\prime \prime}$ the reflections through the sides $P Q, P R$ and $Q R$ respectively $(\bmod \Gamma)$;

(ii) $\rho$ the rotation by $\pi / 4$ centered at $P, \sigma$ the rotation by $\pi$ centered at $Q$ and $\epsilon$ the rotation by $2 \pi / 3$ centered at $R(\bmod \Gamma)$.

Note that $\rho=\kappa^{\prime} \kappa, \sigma=\kappa^{\prime \prime} \kappa$ and $\epsilon=\kappa^{\prime \prime} \kappa^{\prime}$. Moreover $\rho \sigma \epsilon=I d$. Any two of these "rotations" generate the subgroup $\mathcal{G}_{0}$ of orientation-preserving automorphisms of $\mathcal{G}$. It can be seen that $\mathcal{G}=\mathcal{G}_{0} \cup \kappa \cdot \mathcal{G}_{0}$, and moreover $\mathcal{G}_{0}$ can be identified with $G L(2,3)$, the group of invertible $2 \times 2$ matrices with entires in the field $\mathbb{Z}_{3}$. 
Tables 1 and 2 list the conjugacy classes of elements in $\mathcal{G}_{0}$ and $\mathcal{G} \backslash \mathcal{G}_{0}$ respectively. In table 2 we

\begin{tabular}{|c|c|c|c|c|c|c|c|}
\hline class number & 1 & 2 & 3 & 4 & 5 & 6 & 7 \\
\hline representative & $I d$ & $\rho$ & $\rho^{2}$ & $-I d$ & $\sigma$ & $\epsilon$ & $-\epsilon$ \\
\hline order & 1 & 8 & 4 & 2 & 2 & 3 & 6 \\
\hline \# elements & 1 & 12 & 6 & 1 & 12 & 8 & 8 \\
\hline
\end{tabular}

Table 1: Conjugacy classes of $\mathcal{G}$, orientation preserving transformations

\begin{tabular}{|c|c|c|c|c|c|c|}
\hline class number & 8 & 9 & 10 & 11 & 12 & 13 \\
\hline representative & $\kappa$ & $\kappa^{\prime}$ & $\widehat{\sigma} \kappa$ & $\rho \widehat{\sigma} \kappa$ & $\epsilon \kappa$ & $-\epsilon \kappa$ \\
\hline order & 2 & 2 & 8 & 4 & 12 & 12 \\
\hline \# elements & 6 & 12 & 12 & 2 & 8 & 8 \\
\hline
\end{tabular}

Table 2: Conjugacy classes of $\mathcal{G}$, orientation reversing transformations

simplify expressions by using the notation $\widehat{\sigma}=\epsilon \sigma \epsilon^{-1}$. We also define $\widetilde{\sigma}=\rho^{2} \sigma \rho^{-2}$. Note that $\widehat{\sigma}$ is the rotation by $\pi$ centered at the point $\widehat{S}(\bmod \Gamma)$ and $\widetilde{\sigma}$ is the rotation by $\pi$ centered at $\widetilde{S}(\bmod \Gamma)$ in Figure 1.

There are 13 conjugacy classes and therefore 13 complex irreducible representations of $\mathcal{G}$, the characters of which will be denoted $\chi_{j}, j=1, \ldots, 13$. The character table, as computed by the group algebra software GAP is shown in table 3 (GAP, http://www.gap-system.org/). The character of the identity is equal to the dimension of the corresponding representation. It follows from table 3 that there are 4 irreducible representations of dimension 1, 2 of dimension 2, 4 of dimension 3 and 3 of dimension 4 . In the following we shall denote the irreducible representations by their character: $\chi_{j}$ is the representation with this character. The following lemma is proved in [14].

Lemma 1. All the irreduclible representations of $\mathcal{G}$ are real absolutely irreducible. In other words, any matrix which commutes with such a representation is a real scalar multiple of the identity matrix.

\subsection{Steady-state bifurcations with $\mathcal{G}$ symmetry: earlier results}

We shall assume throughout the paper that a center manifold reduction has been performed for a steadystate bifurcation problem with $\mathcal{G}$ symmetry as can arise from Equation (2) restricted to $\Gamma$-periodic patterns in $\mathcal{D}$, where $I_{\text {ext }}=0$ (no external input). This means that a linear stability analysis of the trivial solution has led to finding a critical parameter value $\mu_{c}$ at which, in the class of $\Gamma$-periodic functions, 0 is an eigenvalue of the linear part. The existence of such critical points is discussed in [14] and [13]. It is a generic fact that the corresponding eigenspace $X$ be an irreducible representation space of $\mathcal{G}$, and any irreducible representation can be involved, depending on the form of the function $w$ defined in the introduction in (2). Then the center manifold theorem reduces the initial problem to an ODE 


\begin{tabular}{|c|c|c|c|c|c|c|c|c|c|c|c|c|c|}
\hline Class \# & 1 & 2 & 3 & 4 & 5 & 6 & 7 & 8 & 9 & 10 & 11 & 12 & 13 \\
\hline Representative & $I d$ & $\rho$ & $\rho^{2}$ & $-I d$ & $\sigma$ & $\epsilon$ & $-\epsilon$ & $\kappa$ & $\kappa^{\prime}$ & $\widehat{\sigma} \kappa$ & $\rho \widehat{\sigma} \kappa$ & $\epsilon \kappa$ & $-\epsilon \kappa$ \\
\hline \hline$\chi_{1}$ & 1 & 1 & 1 & 1 & 1 & 1 & 1 & 1 & 1 & 1 & 1 & 1 & 1 \\
\hline$\chi_{2}$ & 1 & -1 & 1 & 1 & -1 & 1 & 1 & 1 & -1 & -1 & 1 & 1 & 1 \\
\hline$\chi_{3}$ & 1 & -1 & 1 & 1 & -1 & 1 & 1 & -1 & 1 & 1 & -1 & -1 & -1 \\
\hline$\chi_{4}$ & 1 & 1 & 1 & 1 & 1 & 1 & 1 & -1 & -1 & -1 & -1 & -1 & -1 \\
\hline$\chi_{5}$ & 2 & 0 & 2 & 2 & 0 & -1 & -1 & -2 & 0 & 0 & -2 & 1 & 1 \\
\hline$\chi_{6}$ & 2 & 0 & 2 & 2 & 0 & -1 & -1 & 2 & 0 & 0 & 2 & -1 & -1 \\
\hline$\chi_{7}$ & 3 & 1 & -1 & 3 & -1 & 0 & 0 & -1 & -1 & 1 & 3 & 0 & 0 \\
\hline$\chi_{8}$ & 3 & 1 & -1 & 3 & -1 & 0 & 0 & 1 & 1 & -1 & -3 & 0 & 0 \\
\hline$\chi_{9}$ & 3 & -1 & -1 & 3 & 1 & 0 & 0 & 1 & -1 & 1 & -3 & 0 & 0 \\
\hline$\chi_{10}$ & 3 & -1 & -1 & 3 & 1 & 0 & 0 & -1 & 1 & -1 & 3 & 0 & 0 \\
\hline$\chi_{11}$ & 4 & 0 & 0 & -4 & 0 & -2 & 2 & 0 & 0 & 0 & 0 & 0 & 0 \\
\hline$\chi_{12}$ & 4 & 0 & 0 & -4 & 0 & 1 & -1 & 0 & 0 & 0 & 0 & $\sqrt{3}$ & $-\sqrt{3}$ \\
\hline$\chi_{13}$ & 4 & 0 & 0 & -4 & 0 & 1 & -1 & 0 & 0 & 0 & 0 & $-\sqrt{3}$ & $\sqrt{3}$ \\
\hline
\end{tabular}

Table 3: Irreducible characters of $\mathcal{G}$

posed in $X$, which is invariant under the action of the irreducible representation of $\mathcal{G}$ in $X$. See [26] for a complete and rigorous exposition of the method and [11] for an exposition in the context of equivariant bifurcations.

Here it may be useful to recall some basic facts about bifurcations with symmetry. We write the bifurcation equation in $X$

$$
\frac{d x}{d t}=\alpha(\lambda) x+f(x, \lambda)
$$

where $\lambda=\mu-\mu_{c}$ and $\alpha$ is a real $C^{k}$ function $(k \geq 1)$ with $\alpha(0)=0, f: X \times \mathbb{R} \rightarrow X$ has order $\|x\| o(\|x\|)$ and commutes with the action of $\mathcal{G}$ in $X$ : if we denote by $(g, x) \mapsto g \cdot x$ the action (representation) of the group in $X$, then $f(g \cdot x, \lambda)=g \cdot f(x, \lambda)$ for all triples $(g, x, \lambda)$. Moreover $\alpha^{\prime}(0)=\mu_{c}^{-1}>0$ (see [42]) so that the trivial solution looses stability when $\lambda>0$. This implies that after a suitable change of variable we can replace $\alpha(\lambda)$ by $\lambda$ in (5).

The problem is now to find the non trivial solutions $(x(\lambda), \lambda)$ of $(5)$ such that $x(0)=0$ and to analyze the local dynamics. Let $H$ be an isotropy subgroup of $\mathcal{G}: H=\{g \in \mathcal{G} \mid g \cdot x=x\}$ for some point $x \in X$. We define the fixed point subspace of $H$, or subspace of $H$ symmetry, as

$$
X^{H}=\{x \in X \mid H \cdot x=x\} .
$$

Then for all $h \in H$ we have that $h \cdot f(x, \lambda)=f(h \cdot x, \lambda)=f(x, \lambda)$. Hence $X^{H}$ is invariant under the flow generated by (5): if $x(0) \in X^{H}$, then $x(t) \in X^{H}$ for all $t$. We shall later use the notation $\operatorname{Fix}(H)$ instead of $X^{H}$ for convenience. It follows that by restricting ourselves to the search of solutions with a given isotropy $H$, we just need to solve (5) in the fixed point subspace $X^{H}$. The case when $\operatorname{dim} X^{H}=1$ 
is of particular interest. In this case looking for solutions with isotropy $H$ reduces to solving a scalar bifurcation equation. Under the above assumptions, this equation always has a branch of non trivial, bifurcated equilibria. By group equivariance of the problem any solution generates new solutions by letting $\mathcal{G}$ act on it. There is a one to one correspondance between the number of elements in this $\mathcal{G}$-orbit of solutions and the number of elements in the quotient $\mathcal{G} / H$ (number of subgroups conjugate to $H$ in $\mathcal{G})$. We call the conjugacy class of an isotropy subgroup $H$ the isotropy type of $H$. Moreover, writing $N(H)$ to represent the normalizer of $H$ in $\mathcal{G}$, if $N(H) / H \simeq \mathbb{Z}_{2}$, the branch is generically a pitchfork: $x(\lambda)= \pm O(\sqrt{|\lambda|})$. The above results when $\operatorname{dim} X^{H}=1$ are known as the Equivariant Branching Lemma, see [23], [11]. We may therefore say that if the hypotheses of the Equivariant Branching Lemma are satisfied for a subgroup $H$, then the isotropy type of $H$ is symmetry-breaking.

Note that, when restricted to the invariant axis $X^{H}$ as above, the exchange of stability principle holds for these solutions. Indeed let the axis of symmetry be parametrized by a real coordinate $u$, the equation on this axis at leading order has the form $\dot{u}=\lambda u+C u^{k}$ where $C$ is a real coefficient and $k \geq 2$. The bifurcated branch is parametrized (at leading order) by $\lambda=-C u^{k-1}$, so that the radial eigenvalue is $(k-1) C u^{k-1}=-(k-1) \lambda$ (at leading order). It therefore changes sign with $\lambda$. This eigenvalue is called radial. The other eigenvalues for the Jacobian matrix $J$ of (5) evaluated at the solutions are transverse (the eigenvectors point orthogonally to the axis of symmetry). The bifurcated equilibria are stable in $X$ if the eigenvalues of $J$ all have a negative real part. The exchange of stability principle does not hold in general when considering stability in the full space $X$.

Equilibria with isotropy not satisfying the condition $\operatorname{dim} X^{H}=1$ or other types of bounded solutions may also exist. However their analysis requires knowledge of the equivariant structure of the vector field $f$ or at least of its Taylor expansion up to an order large enough to fully determine the bifurcation diagram. We shall see in the next sections that solving the bifurcation equation (5) when $\operatorname{dim} X=4$ requires computing the equivariant terms in the expansion of $f(\cdot, \lambda)$ up to order 3,5 or 7 depending on the problem treated.

We now come back to our specific problem with $\mathcal{G}$ symmetry. We can see from the character table 3 that there are 13 possible cases for the irreducible representations. The dimension of $X$ for each representation $\chi_{j}(j=1, \ldots, 13)$ is given by the corresponding character evaluated at the identity. We see that $\operatorname{dim}(X)=1$ for $\chi_{1}, \ldots, \chi_{4}, \operatorname{dim}(X)=2$ for $\chi_{5}, \chi_{6}, \operatorname{dim}(X)=3$ for $\chi_{7}, \ldots, \chi_{10}$ and $\operatorname{dim}(X)=4$ for $\chi_{11}, \chi_{12}$ and $\chi_{13}$. In order to give a complete description of the bifurcation diagrams with $\mathcal{G}$ symmetry we have to consider all these 13 cases.

For the representations $\chi_{1}$ to $\chi_{10}$ in Table 3 , it has been established in [14] that the bifurcation diagrams are identical to those of classical bifurcation problems with symmetry in $\mathbb{R}, \mathbb{R}^{2}$ or $\mathbb{R}^{3}$. Let us summarize these cases in the following theorem. We recall that the octahedral group $\mathbb{O}$, the direct 
symmetry group of the cube, possesses two irreducible representations of dimension three. In order to differentiate these two irreducible representations we adopt the convention "natural" and "non natural" as used in [37].

Theorem 1. For generic steady-state bifurcation diagrams with $\mathcal{G}$ symmetry the following holds.

(i) $\chi_{1}$ : transcritical bifurcation (trivial symmetry, exchange of stability holds);

(ii) $\chi_{2}, \chi_{3}, \chi_{4}$ : pitchfork bifurcation $\left(\mathbb{Z}_{2}\right.$ symmetry, exchange of stability holds);

(iii) $\chi_{5}$ : same as bifurcation with hexagonal $D_{6}$ symmetry in the plane;

(iv) $\chi_{6}$ : same as bifurcation with triangular $D_{3}$ symmetry in the plane;

(v) $\chi_{7}$ : same as bifurcation with natural octahedral $\mathbb{O}$ symmetry in $\mathbb{R}^{3}$;

(vi) $\chi_{8}$ : same as bifurcation with natural full octahedral $\mathbb{O} \ltimes \mathbb{Z}_{2}$ symmetry in $\mathbb{R}^{3}$;

(vii) $\chi_{9}$ : same as bifurcation with the (unique) non natural full octahedral symmetry in $\mathbb{R}^{3}$;

(viii) $\chi_{10}$ : same as bifurcation with the (unique) non natural octahedral symmetry in $\mathbb{R}^{3}$.

Moreover in all cases, bifurcated solutions satisfy the Equivariant Branching Lemma and their isotropies are listed in Theorem 5 of [14].

Theorem 5 of [14] gives also the isotropy types of representations $\chi_{11}, \chi_{12}$ and $\chi_{13}$ which have one dimensional fixed-point subspace. Hence by application of the Equivariant Branching Lemma, we know that branches of solutions with these isotropies exist (in a generic sense). However bifurcation diagrams cannot be deduced from already known bifurcation problems. Our aim in the remainder of this paper is to fill this gap. In the next proposition we list these isotropy subgroups which give bifurcated solutions by the Equivariant Branching Lemma. We introduce the following subgroups which will be relevant in the remainder of the paper. We use the notation $\widetilde{\sigma}=\rho^{2} \sigma \rho^{-2}$ (see Table 1).

\section{Definition 2.}

$$
\begin{aligned}
\widetilde{C}_{2 \kappa} & =\langle\sigma, \kappa\rangle=\left\{I d, \sigma, \kappa, \kappa^{\prime \prime}\right\} \\
\widetilde{C}_{2 \kappa}^{\prime} & =\langle\widetilde{\sigma}, \kappa\rangle=\left\{I d, \tilde{\sigma}, \kappa,-\rho^{2} \kappa^{\prime \prime} \rho^{-2}\right\} \\
\widetilde{C}_{3 \kappa^{\prime}} & =\left\langle\epsilon, \kappa^{\prime}\right\rangle=\left\{I d, \epsilon, \epsilon^{2}, \kappa^{\prime}, \epsilon \kappa^{\prime} \epsilon^{2}, \epsilon^{2} \kappa^{\prime} \epsilon\right\} \\
\widetilde{D}_{3} & =\langle\widetilde{\sigma}, \epsilon\rangle=\left\{I d, \epsilon, \epsilon^{2}, \widetilde{\sigma}, \epsilon \widetilde{\sigma} \epsilon^{2}, \epsilon^{2} \widetilde{\sigma} \epsilon\right\}
\end{aligned}
$$

Proposition 1. For the $4 D$ representations of $\mathcal{G}$, the isotropy subgroups with one dimensional fixed point subspace are the following: 
- $\chi_{11}: \widetilde{C}_{2 \kappa}, \widetilde{C}_{2 \kappa}^{\prime}$;

- $\chi_{12}: \widetilde{D}_{3}, \widetilde{C}_{3 \kappa^{\prime}}, \widetilde{C}_{2 \kappa}, \widetilde{C}_{2 \kappa}^{\prime}$;

- $\chi_{13}: \widetilde{D}_{3}, \widetilde{C}_{3 \kappa^{\prime}}, \widetilde{C}_{2 \kappa}, \widetilde{C}_{2 \kappa}^{\prime}$.

These isotropy types are therefore symmetry-breaking.

\subsection{Octagonal H-planforms}

In order to illustrate our purposes, we numerically compute the octagonal H-planforms associated to the isotropy groups given in proposition 1 . We recall that these planforms are eigenfunctions of the Laplace-Beltrami operator in $\mathcal{D}$ which satisfy certain isotropy conditions: (i) being invariant under a lattice group $\Gamma$ and (ii) being invariant under the action of an isotropy subgroup of the symmetry group of the fundamental domain $\mathcal{D} / \Gamma(\bmod \Gamma)$.

Lemma 2. The Laplace-Beltrami operator in $\mathcal{D}$ in $z_{1}, z_{2}$ coordinates is

$$
\Delta_{\mathcal{D}}=\frac{\left(1-z_{1}^{2}-z_{2}^{2}\right)^{2}}{4}\left[\frac{\partial^{2}}{\partial z_{1}^{2}}+\frac{\partial^{2}}{\partial z_{2}^{2}}\right] \text { for } z=z_{1}+\mathbf{i} z_{2} \in \mathcal{D}
$$

In the last section of [14], we tackled the problem of computing octagonal H-planforms and we described the required numerical and geometrical methods. The computations of the four planforms associated to the one-dimensional irreductible representations have been performed using the finite element method (see [15] for a review) on "desymmetrized" domains of the hyperbolic octagon with a mixture of Dirichlet and Neumann boundary conditions as in [5, 4, 41]. The principles of desymmetrization in the context of dihedral symmetry can be found in the book of Fässler and Stiefel [17]. For the two and three dimensional representations, we implemented the finite element method with periodic boundary conditions in the octagon and afterward, identified the corresponding planforms. In this section, we complete this study for the four-dimensional case and we illustrate it with a selection of images of octagonal H-planforms.

We first explain how to recover the desymmetrized domain and the associated boundary conditions for isotropy group $\widetilde{C}_{3 \kappa^{\prime}}$ in Figure 2(a). The group $\widetilde{C}_{3 \kappa^{\prime}}$ has six elements among them are $\epsilon$ the rotation by $2 \pi / 3$ centered at $R$ and the reflections $\kappa^{\prime}, \kappa^{\prime \prime}$ through the side $P R$ and $Q R$ respetively, where $P, Q$ and $R$ are the vertices of the purple triangle in Figure 1. Each reflection implies Neumann boundary conditions on their respective edges. The Dirichlet boundary conditions prevent an additional 3-fold rotation. The last Neumann boundary condition is obtained by translating the desymmetrized domain with the four boosts (4). 


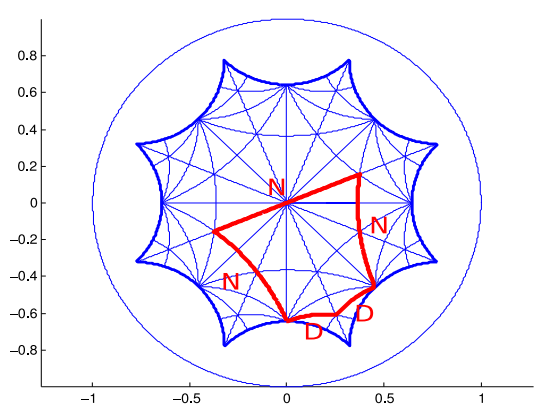

(a) $\widetilde{C}_{3 \kappa^{\prime}}$

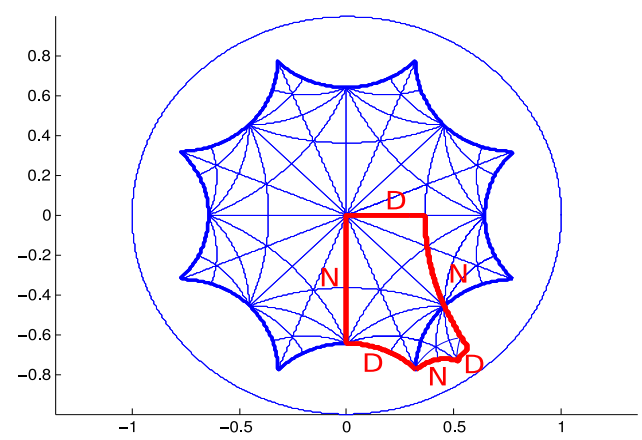

(c) $\bar{C}_{2 \kappa}^{\prime}$

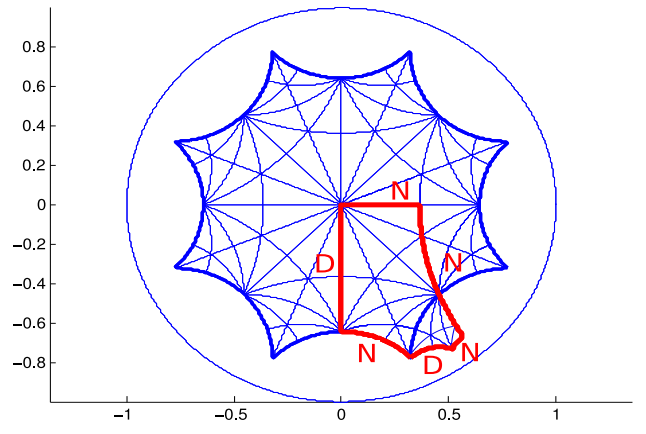

(b) $\widetilde{C}_{2 \kappa}$

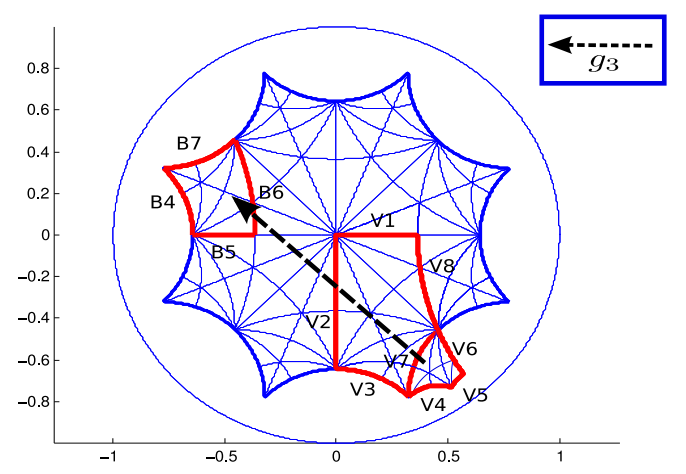

(d) Action of the boost $g_{3}$ on the desymmetrized domain corresponding to isotropy groups $\widetilde{C}_{2 \kappa}$ and $\bar{C}_{2 \kappa}^{\prime}$, see text.

Figure 2: Desymmetrized domain in red and associated boundary conditions corresponding to isotropy groups $\widetilde{C}_{3 \kappa^{\prime}}, \widetilde{C}_{2 \kappa}$ and $\bar{C}_{2 \kappa}^{\prime}$. Letters $N$ and $D$ mean respectively Neumann and Dirichlet boundary conditions. 
In order to better illustrate the intrinsic differences of planforms with isotropy types $\widetilde{C}_{2 \kappa}$ and $\widetilde{C}_{2 \kappa}^{\prime}$, we decide to work with $\bar{C}_{2 \kappa}^{\prime}=\left\{I d,-\sigma,-\kappa, \kappa^{\prime \prime}\right\}$, a conjugate of $\widetilde{C}_{2 \kappa}^{\prime}$. Indeed, isotropy groups $\widetilde{C}_{2 \kappa}$ and $\bar{C}_{2 \kappa}^{\prime}$ share the same desymmetrized domain but have different boundary conditions depending on their symmetries, see Figure 2(b) and 2(c). As we apply finite element method to compute the eigenvalues and eigenvectors of the Laplace-Beltrami operator, it is more convenient to work with a connected domain. This is why the desymmetrized domain of isotropy groups $\widetilde{C}_{2 \kappa}$ and $\bar{C}_{2 \kappa}^{\prime}$ has the particularity of a part outside the octagon, however by the action of the boost $g_{3}$ one can translate this part inside the octagon, see Figure 2(d). Indeed, domain delimited by edges $V 4-V 5-V 6-V 7$ is translated into the domain delimited by edges $B 4-B 5-B 6-B 7$ by the boost $g_{3}$. For isotropy group $\widetilde{C}_{2 \kappa}$, the reflection $\kappa$ imposes Neumann boundary condition on edges $V 1, B 5$ and $\kappa^{\prime \prime}$ on $V 8, V 6$. The action of $g_{3}^{-1}$ implies Neumann boundary condition on edge $V 5=g_{3}^{-1}(B 5)$. We have to impose Dirichlet boundary condition on edge $V 2$ to prevent the action of $-\kappa$, which does not belong to $\widetilde{C}_{2 \kappa}$ and further implies Dirichlet condition on $B 4$ and thus on $V 4$. Finally, reflection $\kappa$ combined with boost $g_{2}$ which translates edge $V 3$ to the opposite side of the octagon, gives Neumann boundary condition on edge $V 3$. The same method applies to isotropy group $\bar{C}_{2 \kappa}^{\prime}$ and we find the boundary conditions presented in Figure 2(c). For isotropy group $\widetilde{D}_{3}$, we do not find any simple desymmetrized domain as in the other cases and we use the finite element method on the full octagon with periodic boundary conditions: opposite sides of the octagon are identified by periodicity. To identify planforms with isotropy group $\widetilde{D}_{3}$, we first select eigenvectors with eigenfunctions of multiplicity 4 and then check the symmetries.

We show in Figure 3 four H-planforms with isotropy groups $\widetilde{C}_{2 \kappa}, \bar{C}_{2 \kappa}^{\prime}, \widetilde{C}_{3 \kappa^{\prime}}$ and $\widetilde{D}_{3}$ with eigenvalue $\lambda=5.3537$ and in Figure 4 two H-planforms with isotropy groups $\widetilde{C}_{2 \kappa}, \bar{C}_{2 \kappa}^{\prime}$. Planform with isotropy group $\widetilde{D}_{3}$ is the only one which does not possess any reflection, it is then easy to distinguish it from other planforms, see Figure 3(d). We notice that patterns of planforms with isotropy $\widetilde{C}_{2 \kappa}$ (in Figure $3(\mathrm{a})$ ) and $\widetilde{C}_{3 \kappa^{\prime}}$ (in Figure 3(c)) appear to be similar up to a rotation, despite the fact that the two cooresponding groups are different. On the contrary, it is easy to distinguish patterns of groups $\widetilde{C}_{2 \kappa}$, Figures $3(\mathrm{a})$ and $4(\mathrm{a})$, and $\bar{C}_{2 \kappa}^{\prime}$, Figures $3(\mathrm{~b})$ and $4(\mathrm{~b})$.

In Figures 3 and 4, we have plotted for convenience, the corresponding H-planforms in the octagon only. Nevertheless, H-planforms are periodic in the Poincaré disc and in Figure 5, we plot the H-planform with $\widetilde{C}_{3 \kappa^{\prime}}$ isotropy type of Figure $3(\mathrm{c})$. As the octagonal lattice group $\Gamma$ is generated by the four boosts of Equation (4), then once an H-planform is computed, we report it periodically in the whole Poincaré disc by the action of these boosts and obtain Figure 5 . 


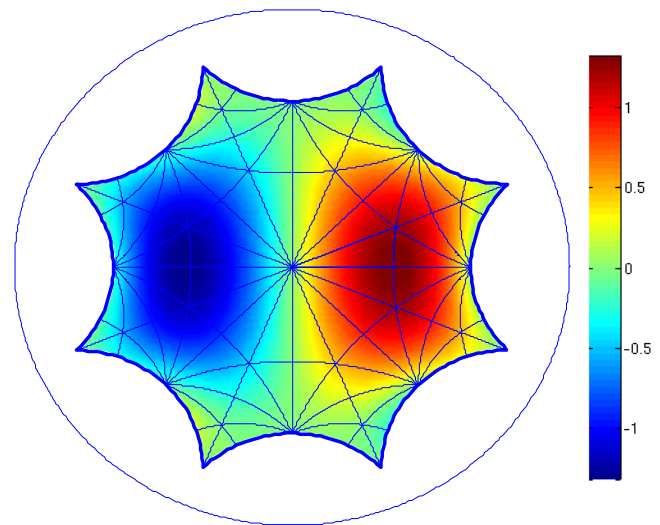

(a) $\widetilde{C}_{2 \kappa}$

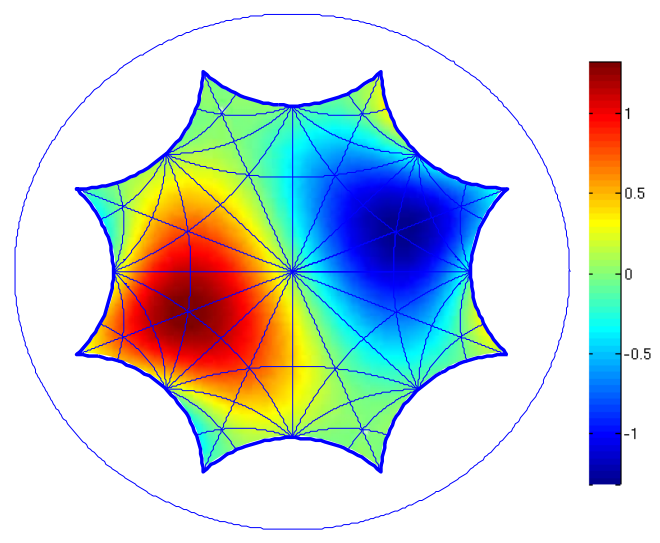

(c) $\widetilde{C}_{3 \kappa^{\prime}}$

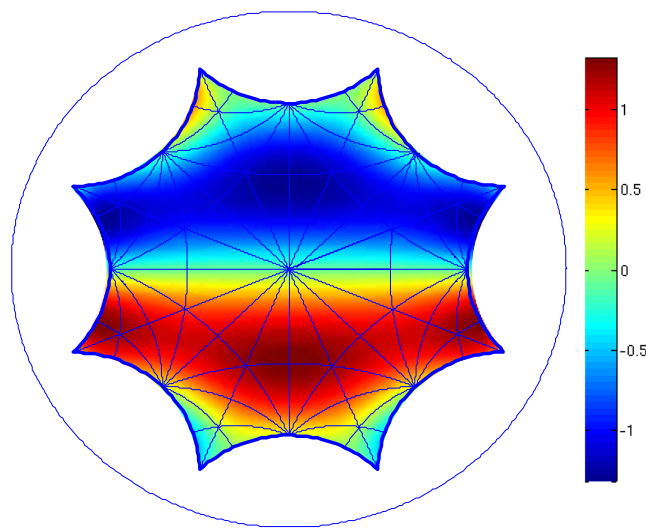

(b) $\bar{C}_{2 \kappa}^{\prime}$

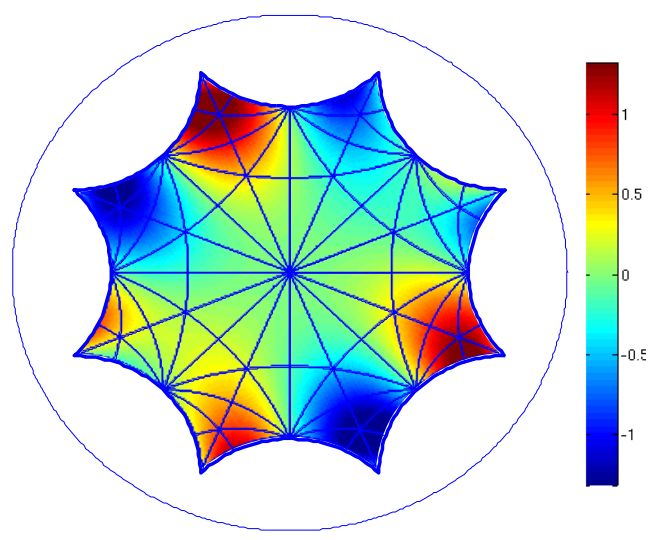

(d) $\widetilde{D}_{3}$

Figure 3: The four H-planforms associated to the eigenvalue $\lambda=5.3537$.

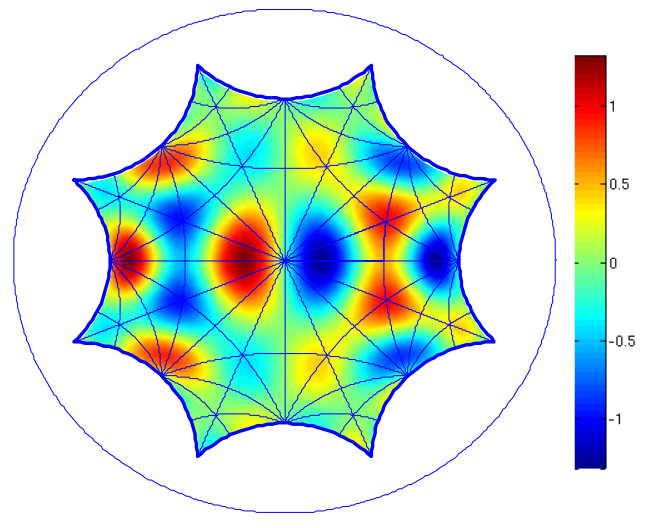

(a) $\widetilde{C}_{2 \kappa}$

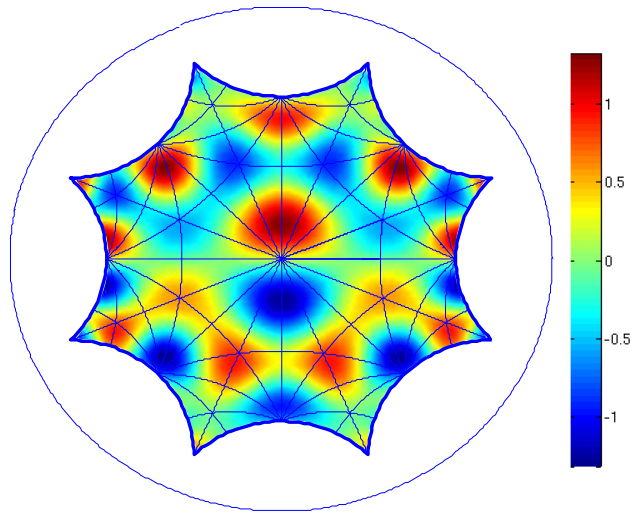

(b) $\bar{C}_{2 \kappa}^{\prime}$

Figure 4: Two H-planforms corresponding to isotropy group $\widetilde{C}_{2 \kappa}$ right and $\bar{C}_{2 \kappa}^{\prime}$ left for eigenvalue $\lambda=$ 42.3695 . 


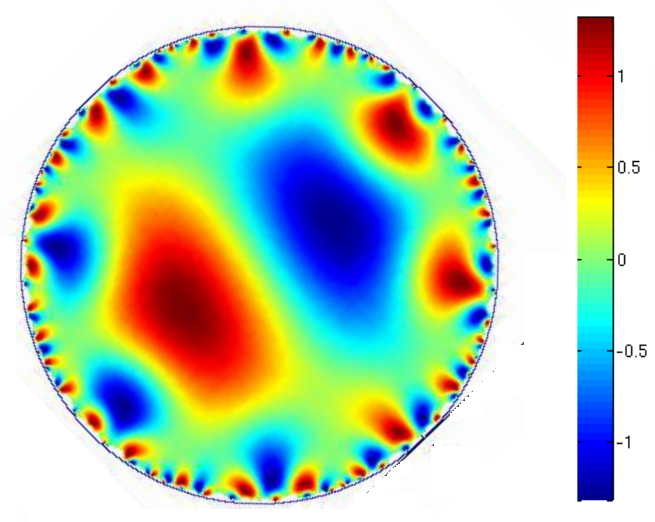

Figure 5: Extension of the $\widetilde{C}_{3 \kappa^{\prime}}$ H-planform on the Poincaré disc of Figure 3(c).

\subsection{Bifurcation with submaximal isotropy}

We briefly recall some basic results on bifurcation with submaximal isotropy which will be used in sections 3 and 4. The condition $\operatorname{dim} X^{H}=1$, required by the Equivariant Branching Lemma, implies that $H$ is maximal (there no isotropy subgroup between $H$ and $\mathcal{G}$ ) and moreover in the case of representations $\chi_{11}, \chi_{12}$ and $\chi_{13}$ there are no maximal isotropy subgroups with fixed-point subspaces of dimension greater than 1 . However it is well-known that solutions with non maximal isotropy can occur in generic bifurcation problems. It follows that the Equivariant Branching Lemma does not account for all the bifurcating equilibria and that the study of bifurcation with submaximal isotropy is an important issue. Here we adopt the approach of [12], see also [11].

The key for the analysis of bifurcation with submaximal isotropy is the determination of the number of copies in $\operatorname{Fix}(\Sigma)$ of subspaces $\operatorname{Fix}(\Delta)$ for the isotropy subgroups $\Delta$ containing $\Sigma$. Let $[\Sigma]$ be the conjugacy classe of $\Sigma$ and write $[\Sigma]<[\Delta]$ if only if $[\Sigma] \neq[\Delta]$ and $\gamma^{-1} \Sigma \gamma \subset \Delta$ for some $\gamma \in \mathcal{G}$. We call isotropy type the conjugacy class of an isotropy subgroup. Let $\left[\Delta_{1}\right], \ldots,\left[\Delta_{r}\right]$ be the isotropy types which satisfy the former condition. Let $a_{j}$ the number of solution branches with isotropy $\Delta_{j}\left(a_{j}\right.$ may be equal to 0$)$. Then the total number of nontrivial solution branches in $\operatorname{Fix}(\Sigma)$ with higher isotropy is

$$
N^{\Sigma}=\sum_{j=1}^{r} a_{j} n\left(\Sigma, \Delta_{j}\right)
$$

where $n\left(\Sigma, \Delta_{j}\right)$ is the number of conjugate copies of $\operatorname{Fix}(\Delta)$ inside $\operatorname{Fix}(\Sigma)$.

We denote $f^{\Sigma}$ the restriction to $\operatorname{Fix}(\Sigma)$ of $f$ given in Eq. (5). Then, if $f^{\Sigma}$ has $N^{0}$ zeroes in a neighborhood of the origin, there are precisely $N^{0}-N^{\Sigma}-1$ branches of equilibria with isotropy $\Sigma$.

If we set $N(\Sigma)=\left\{g \in \mathcal{G} \mid g^{-1} \Sigma g=\Sigma\right\}$ (normalizer of $\Sigma$ in $\mathcal{G}$ ) and $N(\Sigma, \Delta)=\left\{g \in \mathcal{G} \mid \Sigma \subset g \Delta g^{-1}\right\}$, 
the quotient set $\frac{N(\Sigma, \Delta)}{N(\Delta)}$ is well-defined even though $N(\Sigma, \Delta)$ is not a group in general [12]. Moreover we have that

$$
n(\Sigma, \Delta)=\left|\frac{N(\Sigma, \Delta)}{N(\Delta)}\right|
$$

This formula allow us to compute the numbers $n(\Sigma, \Delta)$, hence to determine the number of solutions with isotropy $\Delta$ in $\operatorname{Fix}(\Sigma)$.

Now note that the maximal isotropy subgroups for the representations $\chi_{11}, \chi_{12}$ and $\chi_{13}$ which are listed in Definition 2, have only cyclic subgroups generated by elements $\sigma, \epsilon, \kappa$ or $\kappa^{\prime}$ (or conjugates). The following lemmas give the informations when $\Delta$ is maximal.

Lemma 3. The normalizers of the isotropy subgroups of proposition 1 are listed in table 4.

\begin{tabular}{|c|c|c|}
\hline$\Delta$ & $N(\Delta)$ & $|N(\Delta)|$ \\
\hline$\widetilde{D}_{3}$ & $\left\langle\widetilde{D}_{3},-I d\right\rangle$ & 12 \\
\hline$\widetilde{C}_{3 \kappa^{\prime}}$ & $\left\langle\widetilde{C}_{3 \kappa^{\prime}},-I d\right\rangle$ & 12 \\
\hline$\widetilde{C}_{2 k}$ & $\left\langle\widetilde{C}_{2 k},-I d\right\rangle$ & 8 \\
\hline$\widetilde{C}_{2 \kappa}^{\prime}$ & $\left\langle\widetilde{C}_{2 \kappa}^{\prime},-I d\right\rangle$ & 8 \\
\hline
\end{tabular}

Table 4: Isotropy subgroups of $\mathcal{G}$ and their normalizer. The last column provides the cardinal of the normalizer.

Proof. Let us consider, for example, $\Sigma=\widetilde{C}_{2 k}=\langle\sigma, \kappa\rangle$. The only conjugate of $\kappa$ in $\widetilde{C}_{2 k}$ is $\kappa$ itself, and the same holds for $\sigma$ and $\sigma \kappa=\kappa^{\prime}$. Therefore $N\left(\widetilde{C}_{2 k}\right)=N(\langle\kappa\rangle) \cap N(\langle\sigma\rangle) \cap N(\langle\sigma \kappa\rangle)$. Now for any element $h \in \mathcal{G}$, we have that $|N(\langle h\rangle)|=\frac{96}{|[h]|}$. Tables 1 and 2 show that $|[\kappa]|=6$ while $|[\sigma]|=\left|\left[\kappa^{\prime}\right]\right|=12$. It follows that $|N(\langle\kappa\rangle)|=16$ while $|N(\langle\sigma\rangle)|=\left|N\left(\left\langle\kappa^{\prime}\right\rangle\right)\right|=8$. Hence $|N(\Sigma)| \leq 8$. But $\Sigma \subset N(\Sigma)$ and $|\Sigma|=4$, moreover $-I d$ commutes with any element in $\mathcal{G}$ and therefore belongs to $N(\Sigma)$. It follows that the group $\langle\Sigma,-I d\rangle=N(\Sigma)$. The same rationale applies to the other isotropy subgroups.

Lemma 4. The values of $n(\Sigma, \Delta)$ for the maximal isotropy subgroups $\Delta$ (see Definition 2) are given in table 5 .

Proof. - Case $\Sigma=\langle\kappa\rangle$ : we have $N\left(\Sigma, \widetilde{C}_{2 k}\right)=N\left(\Sigma, \widetilde{C}_{2 k}^{\prime}\right)=N(\Sigma)$ as $\kappa$ is not conjugate to $\sigma$ nor to $\sigma \kappa$. Moreover, $|N(\Sigma)|=16$ (see proof of Lemma 3) and $\left|N\left(\widetilde{C}_{2 k}\right)\right|=\left|N\left(\widetilde{C}_{2 k}^{\prime}\right)\right|=8$, hence $n\left(\Sigma, \widetilde{C}_{2 k}\right)=n\left(\Sigma, \widetilde{C}_{2 k}^{\prime}\right)=2$.

- Case $\Sigma=\langle\sigma\rangle$ : we have $N\left(\Sigma, \widehat{C}_{2 k}\right)=N\left(\Sigma, \widetilde{C}_{2 k}^{\prime}\right)=N(\Sigma)$ with $|N(\Sigma)|=8$ (see proof of Lemma $3)$, hence $n\left(\Sigma, \widehat{C}_{2 k}\right)=n\left(\Sigma, \widetilde{C}_{2 k}^{\prime}\right)=1$. By definition $N\left(\Sigma, \widetilde{D}_{3}\right)=\left\{g \in \mathcal{G} \mid g \Sigma g^{-1} \subset \widetilde{D}_{3}\right\}=\{g \in$ $\left.\mathcal{G} \mid g \widetilde{\sigma} g^{-1} \in \widetilde{D}_{3}\right\}$. There are three conjugates of $\widetilde{\sigma}$ in $\widetilde{D}_{3}$. Therefore $\left|N\left(\Sigma, \widetilde{D}_{3}\right)\right|=3|N(\langle\sigma\rangle)|$. As shown in the proof of Lemma 3$),|N(\langle\sigma\rangle)|=8$, hence $N\left(\Sigma, \widetilde{D}_{3}\right)=24$ and $n\left(\Sigma, \widetilde{D}_{3}\right)=2$. 


\begin{tabular}{|c|c|c|}
\hline$[\Sigma]$ & {$[\Delta]$} & $n(\Sigma, \Delta)$ \\
\hline$\langle\sigma\rangle$ & $\widetilde{C}_{2 k}$ & 1 \\
\hline$\langle\sigma\rangle$ & $\widetilde{C}_{2 k}^{\prime}$ & 1 \\
\hline$\langle\sigma\rangle$ & $\widetilde{D}_{3}$ & 2 \\
\hline$\langle\epsilon\rangle$ & $\widetilde{D}_{3}$ & 2 \\
\hline$\langle\epsilon\rangle$ & $\widetilde{C}_{3 \kappa^{\prime}}$ & 2 \\
\hline$\langle\kappa\rangle$ & $\widetilde{C}_{2 k}$ & 2 \\
\hline$\langle\kappa\rangle$ & $\widetilde{C}_{2 \kappa}^{\prime}$ & 2 \\
\hline$\left\langle\kappa^{\prime}\right\rangle$ & $\widetilde{C}_{2 \kappa}$ & 1 \\
\hline$\left\langle\kappa^{\prime}\right\rangle$ & $\widetilde{C}_{2 \kappa}^{\prime}$ & 1 \\
\hline$\left\langle\kappa^{\prime}\right\rangle$ & $\widetilde{C}_{3 \kappa^{\prime}}$ & 2 \\
\hline
\end{tabular}

Table 5: Values of $n(\Sigma, \Delta)$.

- The proof for the other cases uses the same arguments as above.

\section{Bifurcation diagrams in the case of the representation $\chi_{12}$}

The character table 3 shows that the two $4 \mathrm{D}$ representations $\chi_{12}$ and $\chi_{13}$ are almost identical, the only difference coming from the fact that the characters of the group elements $\epsilon \kappa$ and $-\epsilon \kappa$ have opposite signs. It follows that the general bifurcation analysis in the case of $\chi_{13}$ is identical to the case of $\chi_{12}$ and does not introduce any novelty. In the following we shall therefore only describe the $\chi_{12}$ case.

\subsection{Equivariant structure of the equations on the center manifold}

We need to know the form of the asymptotic expansion of the $\mathcal{G}$ equivariant map $f(\cdot, \lambda)$ in Equation (5). The dimension of the space of equivariant polynomials can be computed using Molien series (see [11]). This computation is shown in appendix B and the results are presented in table 12. The Molien series tells us that there are two independant equivariant homogeneous polynomial maps of order 3 . The computation of these terms will prove to be sufficient to fully determine the bifurcation diagram under generic conditions.

We first need to choose a system of coordinates in $\mathbb{R}^{4}$. In the remaining part of this paper we shall use the same notation for an element in $\mathcal{G}$ and for its representation when there is no ambiguity. The following lemma is proved in Appendix C.1. 
Lemma 5. For the representation $\chi_{12}$ the diagonalization of the 8-fold symmetry matrix $\rho$ has the form

$$
\rho=\left[\begin{array}{cccc}
\exp \left(\frac{\mathbf{i} \pi}{4}\right) & 0 & 0 & 0 \\
0 & \exp \left(-\frac{\mathbf{i} \pi}{4}\right) & 0 & 0 \\
0 & 0 & \exp \left(\frac{3 \mathbf{i} \pi}{4}\right) & 0 \\
0 & 0 & 0 & \exp \left(-\frac{3 \mathbf{i} \pi}{4}\right)
\end{array}\right]
$$

We note $\left(z_{1}, \bar{z}_{1}, z_{2}, \bar{z}_{2}\right)$ the complex coordinates in the corresponding basis.

The following theorem gives the form of the bifurcation equations on the center manifold .

Theorem 2. For the representation $\chi_{12}$, Equation (5) expressed in the coordinates $\left(z_{1}, \bar{z}_{1}, z_{2}, \bar{z}_{2}\right)$ admits the following expansion

$$
\begin{aligned}
& \dot{z}_{1}=\left[\lambda+a\left(\left|z_{1}\right|^{2}+\left|z_{2}\right|^{2}\right)\right] z_{1}+b\left[\sqrt{3}\left(3 z_{1}^{2}+\bar{z}_{2}^{2}\right) \bar{z}_{1}-\mathbf{i}\left(z_{2}^{2}+3 \bar{z}_{1}^{2}\right) z_{2}\right]+\text { h.o.t. } \\
& \dot{z}_{2}=\left[\lambda+a\left(\left|z_{1}\right|^{2}+\left|z_{2}\right|^{2}\right)\right] z_{2}+b\left[\sqrt{3}\left(3 z_{2}^{2}+\bar{z}_{1}^{2}\right) \bar{z}_{2}+\mathbf{i}\left(z_{1}^{2}+3 \bar{z}_{2}^{2}\right) z_{1}\right]+\text { h.o.t. }
\end{aligned}
$$

where $(a, b) \in \mathbb{R}^{2}$. Moreover the cubic part is the gradient of the $\mathcal{G}$ invariant real polynomial function

$$
\frac{a}{2}\left[\left(\left|z_{1}\right|^{2}+\left|z_{2}\right|^{2}\right)^{2}\right]+b \cdot\left[\frac{\sqrt{3}}{2}\left(3\left(z_{1}^{2} \bar{z}_{1}^{2}+z_{2}^{2} \bar{z}_{2}^{2}\right)+z_{1}^{2} z_{2}^{2}+\bar{z}_{1}^{2} \bar{z}_{2}^{2}\right)+\mathbf{i}\left(z_{1}^{3} \bar{z}_{2}+\bar{z}_{2}^{3} z_{1}-z_{2}^{3} \bar{z}_{1}-z_{2} \bar{z}_{1}^{3}\right)\right]
$$

Proof. We postpone to appendix C.1 the computation of the two cubic equivariant maps. The check of the gradient form is straightforward.

\subsection{Isotropy types and fixed points subspaces}

Lemma 6. The lattice of isotropy types for the representation $\chi_{12}$ is shown in Figure 6. The numbers in parentheses indicate the dimension of corresponding fixed-point subspaces.

Proof. We apply the trace formula: if $H$ is a subgroup and $\chi$ is the character of the representation, then

$$
\operatorname{dim}\left(X^{H}\right)=\frac{1}{|H|} \sum_{h \in H} \chi(h)
$$

By applying (8) for $\chi_{12}$ (see Table 3), one finds that the only cyclic subgroups of $\mathcal{G}$ (subgroups generated by one element) with a fixed-point subspace of positive dimension are those listed in the diagram of Figure 6 , and this dimension is equal to 2 . The result follows. Note that the isotropy types with one-dimensional fixed-point subspace have been determined in [14], see Proposition 1. 
The next lemma gives expressions for the fixed-point subspaces of two-element groups in the $\left(z_{1}, \bar{z}_{1}, z_{2}, \bar{z}_{2}\right)$ coordinates, which will be usefull for the bifurcation analysis of (5) in the planes of symmetry. There are four types of these planes but we express the fixed-point planes for the conjugates $\tilde{\sigma}$ of $\sigma$ and $\kappa^{\prime \prime}$ of $\kappa^{\prime}$ for later convenience.

Lemma 7. Fixed-point subspaces associated with the isotropy groups in the diagram 6 have the following equations.

$$
\begin{aligned}
& -F i x(\sigma)=\left\{\left(z_{1}, \bar{z}_{1}, z_{2}, \bar{z}_{2}\right) \mid z_{2}=(\sqrt{2}-1)\left(\sqrt{2} z_{1}-\mathbf{i} \sqrt{3} \bar{z}_{1}\right)\right\} \\
& \text { - } F i x(\tilde{\sigma})=\rho^{2} F i x(\sigma)=\left\{\left(z_{1}, \bar{z}_{1}, z_{2}, \bar{z}_{2}\right) \mid z_{2}=(1-\sqrt{2})\left(\sqrt{2} z_{1}+\mathbf{i} \sqrt{3} \bar{z}_{1}\right)\right\} \\
& \text { - } \operatorname{Fix}(\epsilon)=\left\{\left(z_{1}, \bar{z}_{1}, z_{2}, \bar{z}_{2}\right) \mid z_{2}=(1+\mathbf{i}) z_{1}+\sqrt{3} \bar{z}_{1}\right\} \\
& \text { - } \operatorname{Fix}(\kappa)=\left\{\left(z_{1}, \bar{z}_{1}, z_{2}, \bar{z}_{2}\right) \mid z_{1}=\mathbf{i} \bar{z}_{1} \text { and } z_{2}=\mathbf{i} \bar{z}_{2}\right\} \\
& \text { - } \left.\left.\left.\operatorname{Fix}\left(\kappa^{\prime}\right)=\left\{\left(z_{1}, \bar{z}_{1}, z_{2}, \bar{z}_{2}\right) \mid \sqrt{2} z_{1}=(-1+\mathbf{i}) \bar{z}_{1}\right)\right\} \text { and } \sqrt{2} z_{2}=-(1+\mathbf{i}) \bar{z}_{2}\right)\right\} \\
& \text { - } \operatorname{Fix}\left(\kappa^{\prime \prime}\right)=\left\{\left(z_{1}, \bar{z}_{1}, z_{2}, \bar{z}_{2}\right) \mid z_{2}=(\sqrt{3}-\sqrt{2})\left(-\sqrt{2} z_{1}+\mathbf{i} \bar{z}_{1}\right)\right\}
\end{aligned}
$$

Proof. Given in Appendix D.1.

The one dimensional fixed point subspaces are the intersections of planes of symmetry. This allows to easily obtain expressions for these axes from the expressions listed in Lemma 7. For example we can write

$$
\operatorname{Fix}\left(\widetilde{C}_{2 \kappa}\right)=\left\{\left(z_{1}, z_{2}\right) \in \mathbb{C}^{2} \mid z_{1}=\mathbf{i} \bar{z}_{1} \text { and } z_{2}=(\sqrt{2}-1)(\sqrt{2}-\sqrt{3}) z_{1}\right\}
$$

\subsection{Bifurcation analysis}

Theorem 3. Provided that $(a, b) \in \mathcal{P}=\left\{(a, b) \in \mathbb{R}^{2} \mid 3 a+2 b \sqrt{3}<0\right.$ and $\left.3 a+10 b \sqrt{3}<0\right\}$ (see Figure 7), the following holds for Equations (6)-(7).

(0)

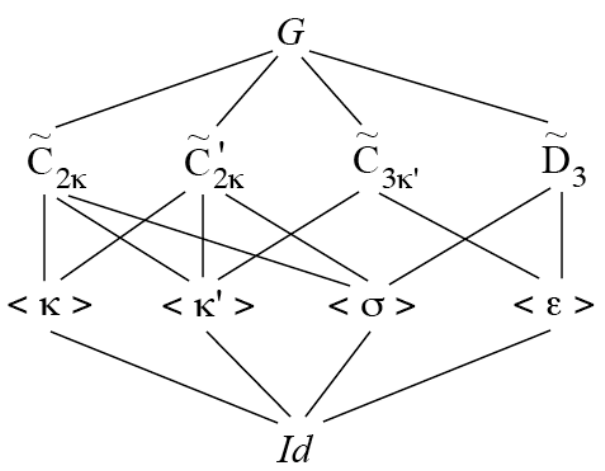

Figure 6: The lattice of isotropy types for the representation $\chi_{12}$. 


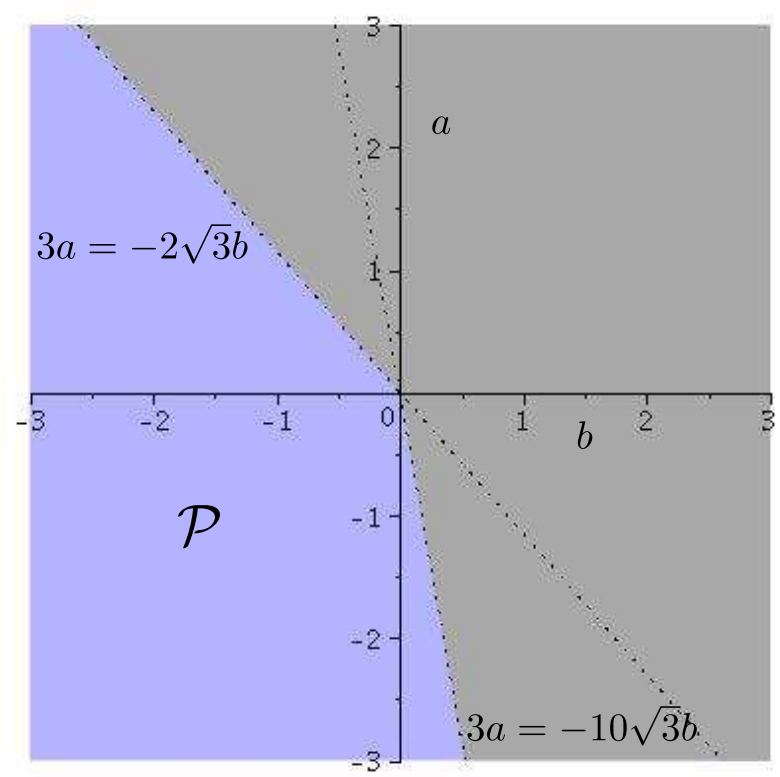

Figure 7: Region $\mathcal{P}=\left\{(a, b) \in \mathbb{R}^{2} \mid 3 a+2 b \sqrt{3}<0\right.$ and $\left.3 a+10 b \sqrt{3}<0\right\}$ for the value of the parameters $(a, b)$ is colored in blue.

(i) No solution with submaximal isotropy bifurcates in the planes of symmetry.

(ii) The branches of equilibria with maximal isotropy (as listed in Proposition 1) are pitchfork and supercritical.

(iii) If $b>0($ resp. $b<0)$, the equilibria with isotropy type $\widetilde{C}_{3 \kappa^{\prime}}$ (resp. $\left.\widetilde{D}_{3}\right)$ are stable in $\mathbb{R}^{4}$. Branches with isotropy $\widetilde{C}_{2 \kappa}$ and $\widetilde{C}_{2 \kappa}^{\prime}$ are always saddles.

Remark 1. We have numerically checked that the domain $\mathcal{P}$ coincides with the existence of an attracting, flow invariant sphere homeomorphic to $S^{3}$ in $\mathbb{R}^{4}$. By a theorem due to Field [19, 11], a condition for the existence of such a sphere is that $\langle q(\xi), \xi\rangle<0$ for all $\xi \neq 0$, where $\xi=\left(z_{1}, \bar{z}_{1}, z_{2}, \bar{z}_{2}\right)$, q is the cubic part in the equations (6), (7) and $\langle$,$\rangle denotes the inner product \Re\left(z_{1} \bar{z}_{1}^{\prime}+z_{2} \bar{z}_{2}^{\prime}\right)$. Since $q$ is an homogeneous polynomial map, it is sufficient to check the condition for $\left(z_{1}, z_{2}\right) \in S^{3}$, which does not present any difficulty.

Remark 2. The theorem doesn't rule out the possibility that equilibria with trivial isotropy could bifurcate. We conjecture this is not the case. This is supported by the fact that under the "generic" hypotheses of the theorem: (i) no other solution than those with maximal isotropy bifurcates in the planes of symmetry, (ii) the stability of these solutions is determined at cubic order and one of these types is always stable, (iii) the system is gradient at cubic order, (iv) admitting the existence of an invariant sphere (previous remark), the conjecture doesn't contradict Poincaré-Hopf formula [25]: one can check that the sum of indices of equilibria with maximal isotropy is equal to 0 , the Euler characteristic of $S^{3}$. 
Proof. We first compute the branches with maximal isotropy, then we examine bifurcation in the invariant planes and finally we provide the eigenvalues of the bifurcated equilibria to complete the stability diagrams.

1. Branches with maximal isotropy. Notice that, since $-I d$ acts non trivially in $\mathbb{R}^{4}-\{0\}$ for $\chi_{12}$, equilibria have to occur via pitchork bifurcations. The maximal isotropy subgroups are $\widetilde{C}_{2 \kappa}, \widetilde{C}_{2 \kappa}^{\prime}, \widetilde{C}_{3 \kappa^{\prime}}$ and $\widetilde{D}_{3}$. By Lemma 7 one can easily find the following parametrizations. Pluging this into the system (6)-(7) we obtain scalar equations which we solve for the bifurcated branches:

- $\operatorname{Fix}\left(\widetilde{C}_{2 \kappa}\right)=\left\{z_{1}=(1+\mathbf{i}) x, z_{2}=(\sqrt{2}-1)\left(\sqrt{2} z_{1}-\mathbf{i} \sqrt{3} \bar{z}_{1}\right)\right\}$.

Bifurcated branch: $\lambda=-4 K_{1}(a+2 b(1+\sqrt{3})) x^{2}+O\left(x^{4}\right)$, where $K_{1}=8-3 \sqrt{6}-5 \sqrt{2}+4 \sqrt{3}>0$.

- $\operatorname{Fix}\left(\widetilde{C}_{2 \kappa}^{\prime}\right)=\left\{z_{1}=(1+\mathbf{i}) x, z_{2}=(1-\sqrt{2})\left(\sqrt{2} z_{1}+\mathbf{i} \sqrt{3} \bar{z}_{1}\right)\right\}$.

Bifurcated branch: $\lambda=-4 K_{2}(a+2 b(\sqrt{3}-1)) x^{2}+O\left(x^{4}\right)$, where $K_{2}=8+3 \sqrt{6}-5 \sqrt{2}-4 \sqrt{3}>0$.

- $\operatorname{Fix}\left(\widetilde{C}_{3 \kappa^{\prime}}\right)=\left\{z_{1}=(1+\mathbf{i}(1+\sqrt{2})) x, z_{2}=(1+\mathbf{i}) z_{1}+\sqrt{3} \bar{z}_{1}\right\}$.

Bifurcated branch: $\lambda=-4 K_{3}(a \sqrt{3}+10 b) x^{2}+O\left(x^{4}\right)$, where $K_{3}=-2+\sqrt{6}-2 \sqrt{2}+2 \sqrt{3}>0$.

- $\operatorname{Fix}\left(\widetilde{D}_{3}\right)=\left\{z_{1}=\left(1+\mathbf{i} \frac{1+\sqrt{6}-\sqrt{3}}{-3+\sqrt{2}+\sqrt{3}}\right) x, z_{2}=(1+\mathbf{i}) z_{1}+\sqrt{3} \bar{z}_{1}\right\}$

Bifurcated branch: $\lambda=-4 K_{4}(a \sqrt{3}+2 b) x^{2}+O\left(x^{4}\right)$, where $K_{4}=6 \sqrt{3}+4 \sqrt{6}+10+7 \sqrt{2}>0$.

From the formulas for the branches we deduce the direction of branching of the equilibria, hence their stability along their axes of symmetry. For example the equilibria with $\widetilde{C}_{2 \kappa}$ isotropy bifurcate supercriticaly if $a+2 b(1+\sqrt{3})<0$, and therefore the principle of exchange of stability (between the trivial state and the bifurcated one) holds against perturbations with the same isotropy.

2. Bifurcation in the planes of symmetry. In each of the planes of symmetry there are precisely 4 axes of symmetry. This immediately follows from Table 5. For example $\operatorname{Fix}(\langle\sigma\rangle)$ contains one copy of $\operatorname{Fix}\left(\widetilde{C}_{2 \kappa}\right)$, one copy of $\operatorname{Fix}\left(\widetilde{C}_{2 \kappa}^{\prime}\right)$ and two copies of $\operatorname{Fix}\left(\widetilde{D}_{3}\right)$. Let us choose real coordinates $(x, y)$ in a plane of symmetry $P$ and write the equations in $P$

$$
\begin{aligned}
& \dot{x}=\lambda x+q_{1}(x, y)+\text { h.o.t. } \\
& \dot{y}=\lambda y+q_{2}(x, y)+\text { h.o.t. }
\end{aligned}
$$

where $q_{1}$ and $q_{2}$ are the components of the cubic part in the Taylor expansion of $f$ restricted to $P$. If $(x(\lambda), y(\lambda))$ is a branch of equilibria of this system, then the equation

$$
Q(x, y)=y q_{1}(x, y)-x q_{2}(x, y)=0
$$


admits an axis of solutions $\epsilon\left(x_{0}, y_{0}\right)$ where $\left(x_{0}, y_{0}\right)$ represents the leading order in the Taylor expansion of the solution. If $Q$ is not degenerate the number of such axes is bounded by the degree of $Q$ which is equal to 4 . Now, there are 4 axes of symmetry in $P$ and each of them corresponds to an axis of solutions of the above equation. Therefore if $Q$ is not degenerate, there are no other invariant axes for Equation (11). To prove that there are no submaximal branches of solutions in the planes of symmetry it remains to check the non degeneracy of $Q$.

Calculations with Maple have shown that in all cases the form $Q$ in non degenerate, hence there are no generic bifurcations of solutions with submaximal isotropy in these planes.

Remark. The stability of the bifurcated equilibria in the planes of symmetry is determined by the sign of the eigenvalues of the Jacobian matrix $\left(\begin{array}{ll}\partial_{x} \dot{x} & \partial_{y} \dot{x} \\ \partial_{x} \dot{y} & \partial_{y} \dot{y}\end{array}\right)$ evaluated at the equilibria. One eigenvalue is radial with leading part $-2 \lambda$ (since $q_{1}$ and $q_{2}$ are cubic), the other one is transverse (see Section 2.2). Calculations lead to the Table 6 for the transverse eigenvalues.

\section{Stability in $\mathbb{R}^{4}$.}

Table 6 shows the leading part of the transverse eigenvalues for the two types of bifurcated equilibria. Each equilibrium lies at the intersection of three planes. The four constants $\left(C_{i}\right)_{i=1 \ldots 4}$ in Table 6 are given by:

$$
\begin{aligned}
& C_{1}=10+6 \sqrt{3}-4 \sqrt{6}-7 \sqrt{2}>0 \quad C_{2}=-10+6 \sqrt{3}-4 \sqrt{6}+7 \sqrt{2}>0 \\
& C_{3}=2+2 \sqrt{3}-2 \sqrt{2}-\sqrt{6}>0 \quad C_{4}=-2+2 \sqrt{3}+2 \sqrt{2}-\sqrt{6}>0
\end{aligned}
$$

For those with $\widetilde{C}_{2 \kappa}$ and $\widetilde{C}_{2 \kappa}^{\prime}$ isotropy, the planes are $\operatorname{Fix}(\kappa), \operatorname{Fix}(\sigma)$ and $\operatorname{Fix}\left(\kappa^{\prime}\right)$. The equilibria with $\widetilde{C}_{3 \kappa^{\prime}}$ isotropy lie in $\operatorname{Fix}(\epsilon)$ and $\operatorname{Fix}\left(\kappa^{\prime}\right)$. The action of $\epsilon$ "rotates" $\operatorname{Fix}\left(\kappa^{\prime}\right)$ by an angle $2 \pi / 3$ around the axis $\operatorname{Fix}\left(\widetilde{C}_{3 \kappa^{\prime}}\right)$, hence a transverse eigenvalue is double and has eigenvectors in $\operatorname{Fix}\left(\kappa^{\prime}\right)$ and its copies by $\epsilon$ and $\epsilon^{2}$. Similarly, equilibria with $\widetilde{D}_{3}$ isotropy have a double transverse eigenvalue with eigenvectors in $\operatorname{Fix}(\sigma)$ and its copies by $\epsilon$ and $\epsilon^{2}$.

\begin{tabular}{|c|c|c|c|c|}
\hline & $\widetilde{C}_{2 \kappa}$ & $\widetilde{C}_{2 \kappa}^{\prime}$ & $\widetilde{C}_{3 \kappa^{\prime}}$ & $\widetilde{D}_{3}$ \\
\hline $\operatorname{Fix}(\kappa)$ & $-32 K_{1} b X^{2}$ & $32 K_{2} b X^{2}$ & no & no \\
\hline $\operatorname{Fix}(\epsilon)$ & no & no & $-64 K_{3} b X^{2}$ & $64 K_{4} b X^{2}$ \\
\hline $\operatorname{Fix}(\sigma)$ & $-16 C_{1} b X^{2}$ & $-16 C_{2} b X^{2}$ & no & $16 K_{4} b X^{2}(2)$ \\
\hline $\operatorname{Fix}\left(\kappa^{\prime}\right)$ & $16 C_{3} b X^{2}$ & $16 C_{4} b X^{2}$ & $-16 K_{3} b X^{2}(2)$ & no \\
\hline
\end{tabular}

Table 6: Transverse eigenvalues of bifurcated equilibria in $\mathbb{R}^{4}$.

Now, Table 6 shows that equilibria with isotropies $\widetilde{C}_{2 \kappa}$ and $\widetilde{C}_{2 \kappa}^{\prime}$ are never stable. Indeed their transverse eiganvalues in the planes $\operatorname{Fix}(\sigma)$ and $\operatorname{Fix}\left(\kappa^{\prime}\right)$ have opposite signs (we assume the generic 
condition $b \neq 0$ to be true). Now suppose that the solutions with isotropy $\widetilde{D}_{3}$ are supercritical, a condition which is fulfilled if $a \sqrt{3}+2 b<0$. Their transverse eigenvalues have the same sign as $b$. It follows that if $b<0$ these solutions are stable (while all other equilibria are unstable).

The same argument applies to solutions with isotropy $\widetilde{C}_{3 \kappa^{\prime}}$ : if $a \sqrt{3}+10 b<0$ (supercritical branch) and $b>0$, these solutions are stable in $\mathbb{R}^{4}$ while all other equilibria are unstable.

It remains to check the domain $\mathcal{P}$ in the theorem. One can easily show that all bifurcated branches are supercritical if the inequalities $a \sqrt{3}+2 b<0$ and $a \sqrt{3}+10 b<0$ are satisfied. This finishes the proof.

\section{Bifurcation diagrams in the case of the representation $\chi_{11}$}

\subsection{Equivariant structure of the equations on the center manifold}

As for representation $\chi_{12}$, we also need to know the form of the asymptotic expansion of $f(\cdot, \lambda)$ in Equation (5). Table 12 of appendix B, given by the computation of Molien series, shows that there are only one equivariant homogeneous polynomial map of order 3 and four linearily independant equivariant maps of order 5. The bifurcation diagrams are fully determined, under generic conditions, by the computations of these terms. However, it turns out that these computations are not anymore sufficient if one wants to study some specific dynamics of Equation (5) as depicted in section 5.

We first need to choose a system of coordinates in $\mathbb{R}^{4}$. The following lemma is proved in Appendix C.2.

Lemma 8. For the representation $\chi_{11}$ the diagonalization of the 8-fold symmetry matrix $\rho$ has the form

$$
\rho=\left[\begin{array}{cccc}
\exp \left(\frac{\mathbf{i} \pi}{4}\right) & 0 & 0 & 0 \\
0 & \exp \left(-\frac{\mathbf{i} \pi}{4}\right) & 0 & 0 \\
0 & 0 & \exp \left(\frac{3 \mathbf{i} \pi}{4}\right) & 0 \\
0 & 0 & 0 & \exp \left(-\frac{3 \mathbf{i} \pi}{4}\right)
\end{array}\right]
$$

We note $\left(z_{1}, \bar{z}_{1}, z_{2}, \bar{z}_{2}\right)$ the complex coordinates in the corresponding basis.

Remark 3. The diagonal matrix is the same as in Lemma 5, however the corresponding bases differ for the two representations $\chi_{12}, \chi_{11}$. Indeed, from Propositions 2 and 3 of Appendix A, one can check that the presentation given by biquaternions of $\rho$ for representation $\chi_{12}$ and $\chi_{11}$ are different.

The bifurcation equations of the center manifold is given by the following theorem.

Theorem 4. For the representation $\chi_{11}$, Equation (5) expressed in the coordinates $\left(z_{1}, \bar{z}_{1}, z_{2}, \bar{z}_{2}\right)$ admits the following expansion 


$$
\begin{gathered}
\dot{z}_{1}=\lambda z_{1}+A z_{1}\left(\left|z_{1}\right|^{2}+\left|z_{2}\right|^{2}\right)+a z_{1}\left(\left|z_{1}\right|^{2}+\left|z_{2}\right|^{2}\right)^{2}+b\left(z_{1}^{4} \bar{z}_{2}+4 z_{2}^{3}\left|z_{1}\right|^{2}-z_{2}^{3}\left|z_{2}\right|^{2}\right) \\
+c\left(3 \bar{z}_{1}^{2} z_{2}\left|z_{2}\right|^{2}-z_{1}^{2} \bar{z}_{2}^{3}-2 \bar{z}_{1}^{2}\left|z_{1}\right|^{2} z_{2}\right)+d\left(-5 \bar{z}_{1}^{4} \bar{z}_{2}+\bar{z}_{2}^{5}\right)+\text { h.o.t } \\
\dot{z}_{2}=\lambda z_{2}+A z_{2}\left(\left|z_{1}\right|^{2}+\left|z_{2}\right|^{2}\right)+a z_{2}\left(\left|z_{1}\right|^{2}+\left|z_{2}\right|^{2}\right)^{2}+b\left(-\bar{z}_{1} z_{2}^{4}-4 z_{1}^{3}\left|z_{2}\right|^{2}+z_{1}^{3}\left|z_{1}\right|^{2}\right) \\
+c\left(-3 z_{1} \bar{z}_{2}^{2}\left|z_{1}\right|^{2}+\bar{z}_{1}^{3} z_{2}^{2}+2 z_{1} \bar{z}_{2}^{2}\left|z_{2}\right|^{2}\right)+d\left(5 \bar{z}_{1} \bar{z}_{2}^{4}-\bar{z}_{1}^{5}\right)+\text { h.o.t }
\end{gathered}
$$

where $(A, a, b, c, d) \in \mathbb{R}^{5}$.

Proof. There is one $\mathcal{G}$-equivariant cubic map, hence necessarily equals to $E_{3}(\mathbf{z})=\mathbf{z}\|\mathbf{z}\|^{2}$ with $\mathbf{z}=$ $\left(z_{1}, \bar{z}_{1}, z_{2}, \bar{z}_{2}\right)$. We postpone to Appendix C.2 the computation of the four quintic equivariant maps.

\subsection{Isotropy types and fixed points subspaces}

Lemma 9. The lattice of isotropy types for the representation $\chi_{11}$ is shown in Figure 8. The numbers in parentheses indicate the dimension of corresponding fixed-point subspaces.

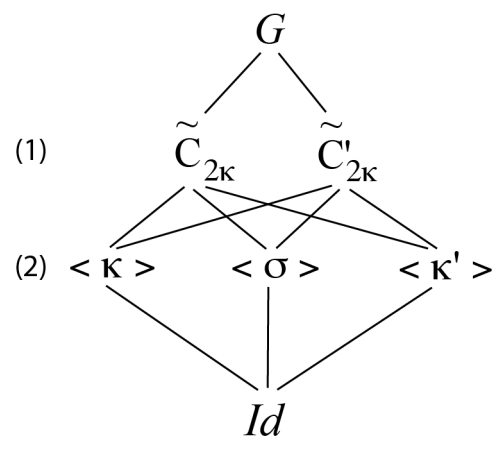

Figure 8: The lattice of isotropy types for the representation $\chi_{11}$.

Proof. The only cyclic subgroups of $\mathcal{G}$ with a fixed-point subspace of positive dimension are given in the diagram of Figure 8 and determined by applying (8) for $\chi_{11}$ (see Table 3). The isotropy types with one-dimensional fixed-point subspace have been determined in [14], see Proposition 1.

The next lemma gives expressions for the fixed-point subspaces of two-element groups in the $\left(z_{1}, \bar{z}_{1}, z_{2}, \bar{z}_{2}\right)$ coordinates, which will be usefull for the bifurcation analysis of (5) in the planes of symmetry. There are three types of these planes. We also express the fixed-point plane for the conjugate $\kappa^{\prime \prime}$ of $\kappa^{\prime}$ for later convenience. 
Lemma 10. Fixed-point subspaces associated with the isotropy groups in the diagram 8 have the following equations.

$$
\begin{aligned}
& -F i x(\sigma)=\left\{\left(z_{1}, \bar{z}_{1}, z_{2}, \bar{z}_{2}\right) \mid z_{2}=\mathbf{i}(1+\sqrt{2}) z_{1}\right\} \\
& -\operatorname{Fix}(\kappa)=\left\{\left(z_{1}, \bar{z}_{1}, z_{2}, \bar{z}_{2}\right) \mid z_{1}=\mathbf{i} \bar{z}_{1} \text { and } z_{2}=-\mathbf{i} \bar{z}_{2}\right\} ; \\
& -\operatorname{Fix}\left(\kappa^{\prime}\right)=\left\{\left(z_{1}, \bar{z}_{1}, z_{2}, \bar{z}_{2}\right) \mid z_{1}=\frac{\sqrt{2}}{2}(\mathbf{i}-1) \bar{z}_{1} \text { and } z_{2}=\frac{\sqrt{2}}{2}(\mathbf{i}+1) \bar{z}_{2}\right\} \\
& -\operatorname{Fix}\left(\kappa^{\prime \prime}\right)=\left\{\left(z_{1}, \bar{z}_{1}, z_{2}, \bar{z}_{2}\right) \mid z_{2}=\mathbf{i} z_{1}-\sqrt{2} \bar{z}_{1}\right\} .
\end{aligned}
$$

Proof. Given in Appendix D.2.

The one dimensional fixed point subspaces are the intersections of planes of symmetry. This allows to easily obtain expressions for these axes from the expressions listed in Lemma 10. For example we can write

$$
\operatorname{Fix}\left(\widetilde{C}_{2 \kappa}\right)=\left\{\left(z_{1}, z_{2}\right) \in \mathbb{C}^{2} \mid z_{1}=\mathbf{i} \bar{z}_{1} \text { and } z_{2}=\mathbf{i}(1+\sqrt{2}) z_{1}\right\}
$$

\subsection{Bifurcation analysis}

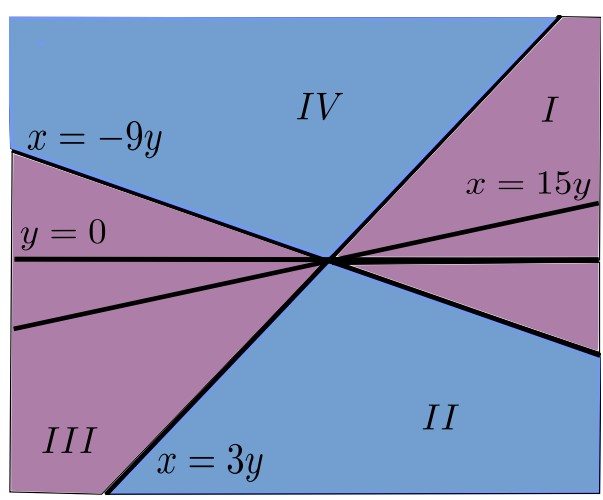

(a) In $\operatorname{Fix}(\sigma)$, bifurcation of submaximal solutions occur for values of coefficients $(b, c, d)$ in the blue regions $I I$ and $I V$. We have set $x=b+c$ and $y=d$.

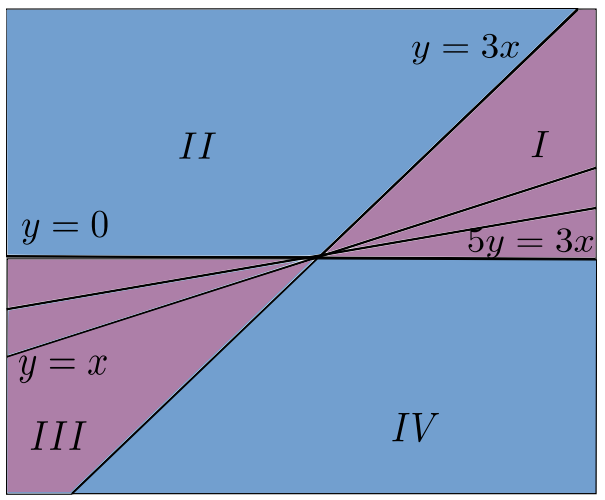

(b) In $\operatorname{Fix}\left(\kappa^{\prime}\right)$, bifurcation of submaximal solutions occur for values of coefficients $(b, c, d)$ in the regions $I I$ and $I V$. We have set $x=c$ and $y=b+d$.

Figure 9: Conditions on coefficients $(b, c, d)$ of existence of submaximal solutions in the planes $\operatorname{Fix}(\sigma)$ and $\operatorname{Fix}\left(\kappa^{\prime \prime}\right)$ (in blue).

Theorem 5. Provided that $A<0$, there exists an attracting, flow invariant, sphere homeomorphic to $S^{3}$ in $\mathbb{R}^{4}$ and branches of equilibria with maximal isotropy (as listed in Proposition 1) are pitchfork and supercritical. The bifurcation diagrams in each fixed-point planes are as follows.

(i) Fix $(\kappa)$ contains two copies of each type of isotropy axes. Moreover,

- No solution with submaximal isotropy bifurcates in Fix( $\kappa)$. 
- If $b<d($ resp. $b>d)$ the equilibria with isotropy $\widetilde{C}_{2 \kappa}$ are stable (resp. saddles) and $\widetilde{C}_{2 \kappa}^{\prime}$ are saddles (resp. stable).

(ii) Fix $(\sigma)$ contains one copy of each type of isotropy axes. Moreover,

- If $b+c+9 d>0($ resp. $b+c+9 d<0)$ equilibria with isotropy $\widetilde{C}_{2 \kappa}$ are stable (resp. saddles) and $\widetilde{C}_{2 \kappa}^{\prime}$ are saddles (resp. stable), see regions I and IV (resp. II and III) in Figure $9(a)$.

- If $d(3 d-b-c)<0$ or $(b+c-15 d)(b+c+9 d)>0$, no solution with submaximal isotropy bifurcates in fixed-point plane Fix $(\sigma)$, see regions I and III in Figure 9(a).

- If $d(3 d-b-c)>0$ and $(b+c-15 d)(b+c+9 d)<0$, regions II and IV in Figure 9(a), solutions with submaximal isotropy bifurcate form equilibria $\widetilde{C}_{2 \kappa}$ and $\widetilde{C}_{2 \kappa}^{\prime}$ in Fix $\left.\sigma\right)$. The corresponding phase diagram is shown in Figure 10.

(iii) Fix $\left(\kappa^{\prime}\right)$ contains one copy of each type of isotropy axes. Moreover,

- If $d+b-3 c<0$ (resp. $d+b-3 c>0)$ equilibria with isotropy $\widetilde{C}_{2 \kappa}$ are stable (resp. saddles) and $\widetilde{C}_{2 \kappa}^{\prime}$ are saddles (resp. stable), see regions II and III (resp. I and IV) in Figure 9(b).

- If $(b+d)(b+d-c)<0$ or $(5 d-3 c+5 b)(d+b-3 c)<0$, no solution with submaximal isotropy bifurcates in fixed-point plane Fix $\left(\kappa^{\prime}\right)$, see regions $I$ and III in Figure $9(b)$.

- If $(b+d)(b+d-c)>0$ or $(5 d-3 c+5 b)(d+b-3 c)>0$, regions $I I$ and $I V$ in Figure $9(b)$, solutions with submaximal isotropy bifurcate form equilibria $\widetilde{C}_{2 \kappa}$ and $\widetilde{C}_{2 \kappa}^{\prime}$ in fixed-point plane Fix $\left(\kappa^{\prime}\right)$. The corresponding phase diagram is given in Figure 10.

Proof. The assumption $A<0$ ensures that $\langle q(\xi), \xi\rangle=A\|\xi\|^{4}<0$ for all $\xi \neq 0$, where $\xi=\left(z_{1}, \bar{z}_{1}, z_{2}, \bar{z}_{2}\right)$, $q$ is the cubic part in the equations (12), (13) and $\langle$,$\rangle denotes the inner product \Re\left(z_{1} \bar{z}_{1}^{\prime}+z_{2} \bar{z}_{2}^{\prime}\right)$ and \|| $\|$ the associated norm. This implies the existence of the invariant sphere homeomorphic to $S^{3}$ in $\mathbb{R}^{4}$. We now examine bifurcation in the invariant planes. We can already note that, since $-I d$ acts non trivially in $\mathbb{R}^{4}-\{0\}$ for $\chi_{12}$, equilibria have to occur via pitchork bifurcations. We proceed as in Section section:chi12 for the case with representation $\chi_{12}$.

1. Branches with maximal isotropy. Since $A<0$ branches of solutions along the axis of symmetry exist for $\lambda>0$ with leading part $\lambda=-A\|X\|^{2}$ where $X$ belongs to the corresponding axis.

2. Bifurcation and stability in $\operatorname{Fix}(\kappa)$. By Lemma 10,

$$
\operatorname{Fix}(\kappa)=\left\{\left(z_{1}, z_{2}\right) \in \mathbb{C}^{2} \mid z_{1}=(1+\mathbf{i}) x \text { and } z_{2}=(1-\mathbf{i}) y, \quad(x, y) \in \mathbb{R}^{2}\right\}
$$




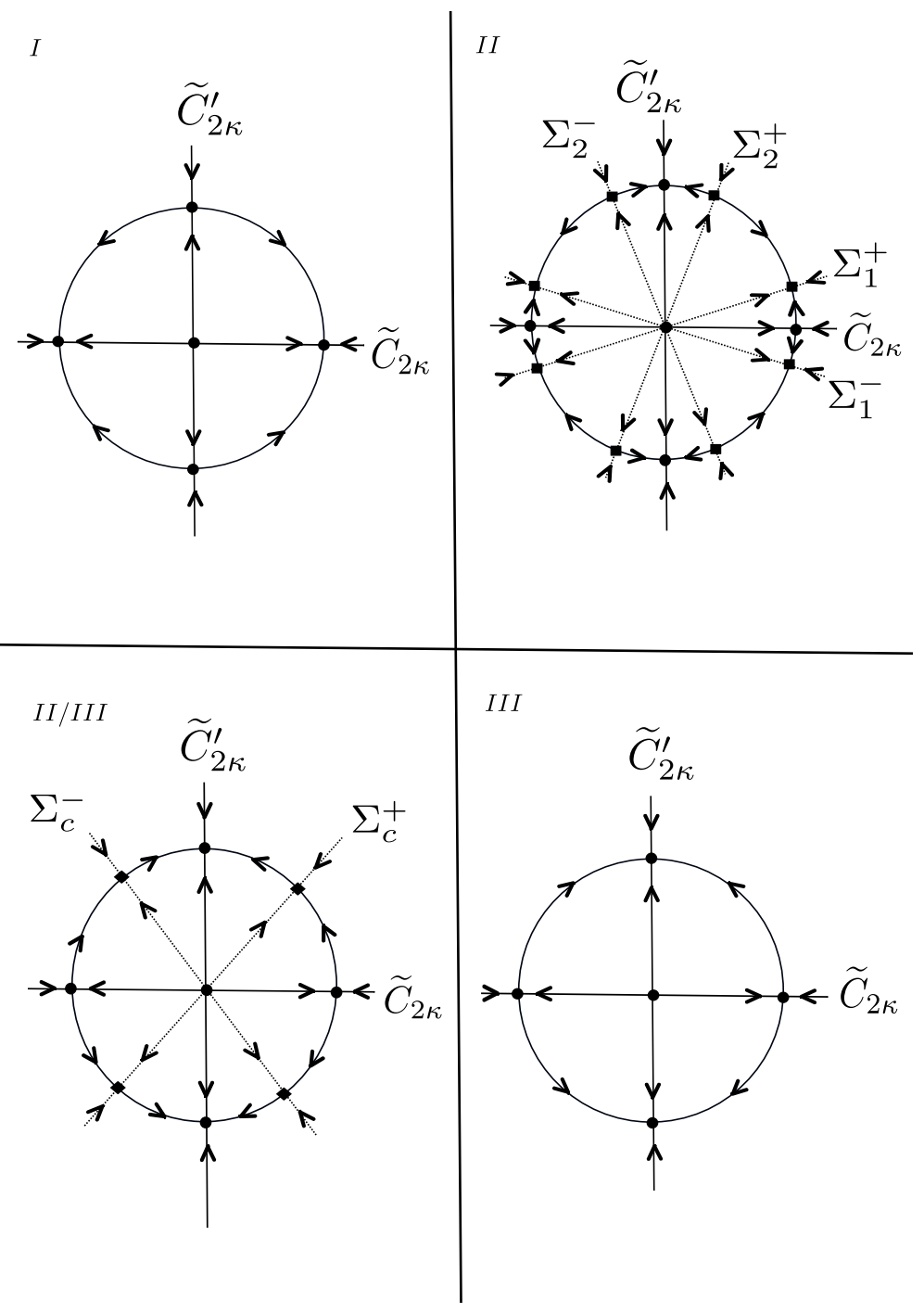

Figure 10: Changes of phase diagram in $\operatorname{Fix}(\sigma)$ and $\operatorname{Fix}\left(\kappa^{\prime}\right)$ as the coefficients $(b, c, d)$ pass from regions $I-I I-I I I$ in Figure 9(a) (for Fix $(\sigma)$ ) and in Figure 9(b) (for Fix $\left(\kappa^{\prime}\right)$ ). $\Sigma_{1}^{ \pm}$and $\Sigma_{2}^{ \pm}$indicate solutions with submaximal isotropy. The case $I I / I I I$ corresponds to coefficient values at the boundary between region $I I$ and $I I I$ ( saddle-node bifurcation of equilibria with submaximal isotropy). 
By Table 5 this plane contains two axes with isotropy type $\widetilde{C}_{2 \kappa}$ and two axes with isotropy type $\widetilde{C}_{2 \kappa}^{\prime}$. We can choose as representative $\operatorname{Fix}\left(\widetilde{C}_{2 \kappa}\right)=\{y=-(1+\sqrt{2}) x\}$ and $\operatorname{Fix}\left(\widetilde{C}_{2 \kappa}^{\prime}\right)=\{y=(1+\sqrt{2}) x\}$. We change coordinates so that $\operatorname{Fix}\left(\widetilde{C}_{2 \kappa}\right)$ is the real axis. With the following choice:

$$
\left(\begin{array}{l}
x \\
y
\end{array}\right)=\left[\begin{array}{cc}
\alpha^{-1} & \beta^{-1} \\
-\alpha^{-1}(1+\sqrt{2}) & \beta^{-1}(\sqrt{2}-1)
\end{array}\right]\left(\begin{array}{l}
X \\
Y
\end{array}\right)
$$

where $\alpha=\sqrt{4+2 \sqrt{2}}$ and $\beta=\sqrt{4-2 \sqrt{2}}$, the equations (12) and (13) read

$$
\begin{array}{r}
\dot{X}=\lambda X+2 A X\left(X^{2}+Y^{2}\right)+4 a X\left(X^{2}+Y^{2}\right)^{2}+2 b X\left(-X^{4}-2 X^{2} Y^{2}+7 Y^{4}\right) \\
+2 c X\left(X^{4}-6 X^{2} Y^{2}+Y^{4}\right)+2 d X\left(3 X^{4}-10 X^{2} Y^{2}-5 Y^{4}\right) \\
\dot{Y}=\lambda Y+2 A Y\left(X^{2}+Y^{2}\right)+4 a Y\left(X^{2}+Y^{2}\right)^{2}-2 b Y\left(-7 X^{4}+2 X^{2} Y^{2}+Y^{4}\right) \\
+2 c Y\left(X^{4}-6 X^{2} Y^{2}+Y^{4}\right)-2 d Y\left(5 X^{4}+10 X^{2} Y^{2}-3 Y^{4}\right)
\end{array}
$$

and the polynomial map $Q$ defined in Equation $(11)$ is $Q(X, Y)=-16(X-Y)(X+Y)\left(X^{2}+\right.$ $\left.Y^{2}\right) X Y(b-d)$. The axes $Y=0$ and $X=0$ correspond to $\widetilde{C}_{2 \kappa}$ isotropy type and the axes $X= \pm Y$ correspond to $\widetilde{C}_{2 \kappa}^{\prime}$ isotropy type. Therefore if $b \neq d$ there are no submaximal solutions in $\operatorname{Fix}(\kappa)$.

Stability of the solutions. The transverse eigenvalues are computed from these equations and are summarized in Table 7.

\begin{tabular}{|c|c|c|}
\hline Isotropy type & $\widetilde{C}_{2 \kappa}$ & $\widetilde{C}_{2 \kappa}^{\prime}$ \\
\hline Tranverse eigenvalue & $16(b-d) X^{4}$ & $-64(b-d) X^{4}$ \\
\hline
\end{tabular}

Table 7: Transverse eigenvalues (leading order) of bifurcated equilibria in Fix $(\kappa)$.

3. Bifurcation and stability in $\operatorname{Fix}(\sigma)$. By Lemma 10,

$$
\operatorname{Fix}(\sigma)=\left\{\left(z_{1}, z_{2}\right) \in \mathbb{C}^{2} \mid z_{2}=\mathbf{i}(1+\sqrt{2}) z_{1}\right\}
$$

By Table 5 there are two axes of symmetry in this plane: one of type $\widetilde{C}_{2 \kappa}$ and one of type $\widetilde{C}_{2 \kappa}^{\prime}$. From Definition 2, a conjugate of $\widetilde{C}_{2 \kappa}^{\prime}$ containing $\sigma$ is $\widetilde{C}_{2 \kappa}^{\prime \prime}=\rho^{-2} \widetilde{C}_{2 \kappa}^{\prime} \rho^{2}=\left\{I d, \sigma,-\kappa,-\kappa^{\prime \prime}\right\}$.

Let us write $z_{1}=x+\mathbf{i} y$. In the $(x, y)$ coordinates, $\operatorname{Fix}\left(\widetilde{C}_{2 \kappa}\right)=\{y=x\}, \operatorname{Fix}\left(\widetilde{C}_{2 \kappa}^{\prime \prime}\right)=\{y=-x\}$. We change coordinates such that $x=\sqrt{2} / 2(X+Y), x=\sqrt{2} / 2(X-Y)$. Hence $Y=0$ is the equation 
of $\operatorname{Fix}\left(\widetilde{C}_{2 \kappa}\right)$ and $X=0$ is the equation of $\operatorname{Fix}\left(\widetilde{C}_{2 \kappa}^{\prime \prime}\right)$. Then

$$
\begin{array}{rl}
\dot{X}=\lambda X+2 E_{1} A X\left(X^{2}+Y^{2}\right)+8 E_{2} a & X\left(X^{2}+Y^{2}\right)^{2}-4 E_{2} b X\left(X^{2}+Y^{2}\right)\left(X^{2}-3 Y^{2}\right) \\
& +4 E_{2} c X\left(X^{2}+Y^{2}\right)^{2}+12 E_{2} d X\left(X^{4}-10 X^{2} Y^{2}+5 Y^{4}\right) \\
\dot{Y}=\lambda Y+2 E_{1} A Y\left(X^{2}+Y^{2}\right)+8 E_{2} a Y & \left(X^{2}+Y^{2}\right)^{2}-4 E_{2} b Y\left(X^{2}+Y^{2}\right)\left(3 X^{2}-Y^{2}\right) \\
- & 4 E_{2} c Y\left(X^{2}+Y^{2}\right)^{2}-12 E_{2} d X\left(5 X^{4}-10 X^{2} Y^{2}+Y^{4}\right)
\end{array}
$$

where $E_{1}=2+\sqrt{2}$ and $E_{2}=3+2 \sqrt{2}$. For this system we obtain

$$
Q(X, Y)=8 E_{2} X Y\left[(b+c+9 d) X^{4}+2(b+c-15 d) X^{2} Y^{2}+(b+c+9 d) Y^{4}\right]
$$

We denote $H(X, Y)=(b+c+9 d) X^{4}+2(b+c-15 d) X^{2} Y^{2}+(b+c+9 d) Y^{4}$.

Study of the polynomial map $H(X, Y)$. We consider $H$ as a polynomial map of degree two in $X^{2}$. When $b+c-15 d=0$, then $H$ is simplified as $H(X, Y)=(b+c+9 d)\left(X^{4}+Y^{4}\right)$, and there is no submaximal bifurcation in $\operatorname{Fix}(\sigma)$. In the remaining part of this paragraph, we suppose that $b+c-15 d \neq 0$.

- Suppose that $d(3 d-b-c)>0$ then $X^{2}=\nu_{ \pm} Y^{2}$ with

$$
\nu_{ \pm}=\frac{-(b+c-15 d) \pm \sqrt{48 d(3 d-b-c)}}{(b+c+9 d)}
$$

and $\nu_{+} \nu_{-}=1, \nu_{+}+\nu_{-}=-2 \frac{b+c-15 d}{b+c+9 d}$. Hence if $\frac{b+c-15 d}{b+c+9 d}<0$, there are four axes $X= \pm \sqrt{\nu_{ \pm}} Y$ which correspond to bifurcated submaximal solutions in $\operatorname{Fix}(\sigma)$. If $\frac{b+c-15 d}{b+c+9 d}>0$ no submaximal bifurcation can bifurcate in $\operatorname{Fix}(\sigma)$.

- Suppose that $d=0$ and $b+c \neq 0$ then $H(X, Y)=(b+c)\left(X^{2}+Y^{2}\right)^{2}$. This implies that no submaximal bifurcation can bifurcate in $\operatorname{Fix}(\sigma)$ if $d=0$ and $b+c \neq 0$.

- If $3 d=b+c \neq 0$ then $H(X, Y)=12 d(X-Y)^{2}(X+Y)^{2}$ and the axes $X= \pm Y$ correspond to bifurcated submaximal solutions in $\operatorname{Fix}(\sigma)$.

- If $d(3 d-b-c)<0$ then $H$ has no other root than $(0,0)$. By the same argument as before, this shows that no submaximal solution can bifurcate in $\operatorname{Fix}(\sigma)$ in this case.

Stability of the solutions. The transverse eigenvalues for isotropy types $\widetilde{C}_{2 \kappa}$ and $\widetilde{C}_{2 \kappa}^{\prime}$ are computed from the above equations and are summarized in Table 8. 


\begin{tabular}{|c|c|c|}
\hline Isotropy type & $\widetilde{C}_{2 \kappa}$ & $\widetilde{C}_{2 \kappa}^{\prime}$ \\
\hline Transverse eigenvalue & $-8(3+2 \sqrt{2})(b+c+9 d) X^{4}$ & $8(3+2 \sqrt{2})(b+c+9 d) X^{4}$ \\
\hline
\end{tabular}

Table 8: Transverse eigenvalues (leading order) of equilibria with maximal isotropy in $\operatorname{Fix}(\sigma)$.

We now discuss the stability of the bifurcated submaximal solutions found for $d(3 d-b-c)>0$ and $(b+c-15 d)(b+c+9 d)<0$, i.e regions $I I$ and $I V$ of Figure 9 (a). We denote $\Sigma_{1}^{+}$(resp. $\Sigma_{1}^{-}$) the branch of solutions with axis $X=\sqrt{\nu_{+}} Y$ (resp. $X=-\sqrt{\nu_{+}} Y$ ) and $\Sigma_{2}^{+}\left(\right.$resp. $\Sigma_{2}^{-}$) the branch of solutions with axis $X=\sqrt{\nu_{-}} Y$ (resp. $X=-\sqrt{\nu_{-}} Y$ ). When parameters pass from region $I$ to region $I I$ in Figure 9(a), pitchfork bifurcation with submaximal isotropy occurs and two equilibria $\Sigma_{1}^{ \pm}$emerge from equilibria with isotropy type $\widetilde{C}_{2 \kappa}$ and two equilibria $\Sigma_{2}^{ \pm}$emerge from equilibria with isotropy type $\widetilde{C}_{2 \kappa}^{\prime}$ with exchange of stability: $\widetilde{C}_{2 \kappa}$ becomes unstable and $\widetilde{C}_{2 \kappa}^{\prime}$ stable, which implies that $\Sigma_{1}^{ \pm}$are stable and $\Sigma_{2}^{ \pm}$are saddles, see Figure 10 (upper right). At the boundary between region $I I$ and $I I I$, equilibria $\Sigma_{1}^{+}$(resp. $\left.\Sigma_{1}^{-}\right)$and $\Sigma_{2}^{+}$(resp. $\Sigma_{2}^{-}$) collide and form only one equilibrium denoted $\Sigma_{c}^{+}\left(\right.$resp. $\Sigma_{c}^{-}$), which no longer exists in region $I I I$ : saddlenode bifurcation. These two equilibria $\Sigma_{c}^{ \pm}$are saddles, see Figure 10 (lower left). In region III, equilibria with isotropy type $\widetilde{C}_{2 \kappa}$ are now unstable whereas equilibria with isotropy type $\widetilde{C}_{2 \kappa}^{\prime}$ are stable, see Figure 10 (lower right). Same phenomena occur when values of the parameters pass from region $I I I$ to region $I$ through region $I V$ in Figure 9(a). We summerize the positive section of the bifurcation diagrams of Figure 10 in Figure 11.

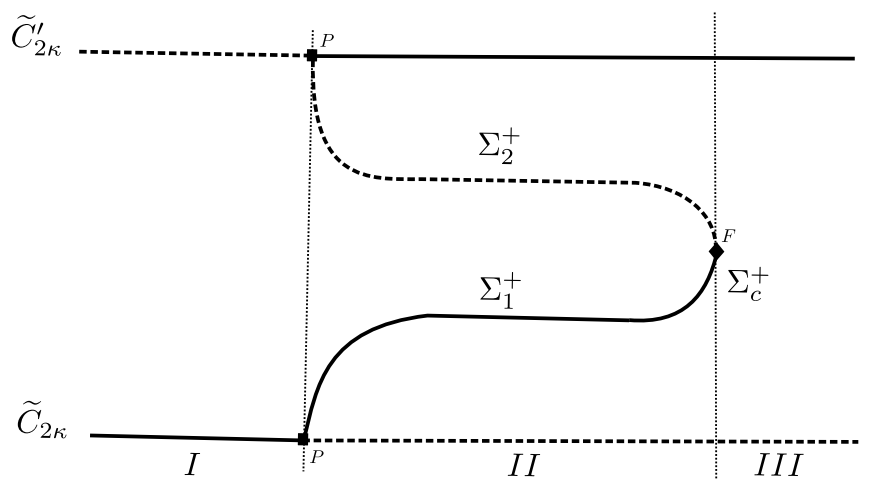

Figure 11: Positive section of the bifurcation diagrams of Figure 10 when parameters pass from regions $I-I I-I I I$. Dashed lines represent unstable branches and continuous lines represent stable branches. 口-P stands for pitchfork bifurcation and $\$$-SN for saddle-node bifurcation, see text for notations. 
4. Bifurcation and stability in $\operatorname{Fix}\left(\kappa^{\prime}\right)$. We consider instead Fix $\left(\kappa^{\prime \prime}\right)$. By Lemma 10,

$$
\operatorname{Fix}\left(\kappa^{\prime \prime}\right)=\left\{\left(z_{1}, \bar{z}_{1}, z_{2}, \bar{z}_{2}\right) \mid z_{2}=\mathbf{i} z_{1}-\sqrt{2} \bar{z}_{1}\right\}
$$

By Table 5 there are two axes of symmetry in this plane: one of type $\widetilde{C}_{2 \kappa}$ and one of type $\widetilde{C}_{2 \kappa}^{\prime}$. We have already noticed that a conjugate of $\widetilde{C}_{2 \kappa}^{\prime}$ which contains $\kappa^{\prime \prime}$ is $\widehat{C}_{2 \kappa}^{\prime}=\left\{I d,-\sigma,-\kappa, \kappa^{\prime \prime}\right\}$. Setting $z_{1}=x+\mathbf{i} y$, the equations for $\operatorname{Fix}\left(\widetilde{C}_{2 \kappa}\right)$ and $\operatorname{Fix}\left(\widehat{C}_{2 \kappa}^{\prime}\right)$ are respectively $y=x$ and $y=-x$. With the change of coordinates $x=\sqrt{2} / 2(X+Y), x=\sqrt{2} / 2(X-Y)$ the equations become:

$$
\begin{aligned}
& \dot{X}=\lambda X+2 E_{1} A X\left(X^{2}+E_{3}^{2} Y^{2}\right)+8 E_{2} a X\left(X^{2}+E_{3}^{2} Y^{2}\right)^{2} \\
& +4 E_{2} b X\left(-X^{2}+2 \sqrt{2} E_{3} X Y+E_{3}^{2} Y^{2}\right)\left(X^{2}+2 \sqrt{2} E_{3} X Y-E_{3}^{2} Y^{2}\right) \\
& +4 E_{2} c X\left(X^{2}-5 E_{3}^{2} Y^{2}\right)\left(X-E_{3} Y\right)\left(X+E_{3} Y\right)+4 E_{2} d X\left(3 X^{4}+10 E_{3}^{2} X^{2} Y^{2}-5 E_{3}^{4} Y^{4}\right) \\
& \dot{Y}=\lambda Y+2 E_{1} A Y\left(X^{2}+E_{3}^{2} Y^{2}\right)+8 E_{2} a Y\left(X^{2}+E_{3}^{2} Y^{2}\right)^{2} \\
& \quad-4 E_{2} b Y\left(-X^{2}+2 \sqrt{2} E_{3} X Y+E_{3}^{2} Y^{2}\right)\left(X^{2}+2 \sqrt{2} E_{3} X Y-E_{3}^{2} Y^{2}\right) \\
& -4 E_{2} c Y\left(5 X^{2}-E_{3}^{2} Y^{2}\right)\left(X-E_{3} Y\right)\left(X+E_{3} Y\right)+4 E_{2} d Y\left(5 X^{4}-10 E_{3}^{2} X^{2} Y^{2}-3 E_{3}^{4} Y^{4}\right)
\end{aligned}
$$

where $E_{3}=1-\sqrt{2}$. Then

$$
Q(X, Y)=-8 E_{2} X Y\left[(b+d-3 c) X^{4}-2 E_{3}^{2}(5 d+5 b-3 c) X^{2} Y^{2}+E_{3}^{4}(b+d-3 c) Y^{4}\right]
$$

We set $K(X, Y)=(b+d-3 c) X^{4}-2 E_{3}^{2}(5 d+5 b-3 c) X^{2} Y^{2}+E_{3}^{4}(b+d-3 c) Y^{4}$.

Study of the polynomial map $K(X, Y)$. We consider $K$ as a polynomial of degre two in $X^{2}$. If $5 d+5 b-3 c=0$, then $K(X, Y)=(b+d-3 c)\left(X^{4}+E_{3}^{4} Y^{4}\right)$, and no submaximal bifurcation can occur in fixed-point plane $\operatorname{Fix}\left(\kappa^{\prime \prime}\right)$. We now suppose that $5 d+5 b-3 c \neq 0$.

- If $(b+d)(b+d-c)>0$ then $X^{2}=\nu_{ \pm} Y^{2}$ with

$$
\nu_{ \pm}=E_{3}^{2} \frac{5 d-3 c+5 b \pm 2 \sqrt{6(b+d)(b+d-c)}}{(d+b-3 c)} Y^{2}
$$

where $\nu_{+} \nu_{-}=C^{2}$ and $\nu_{+}+\nu_{-}=2 E_{3}^{2} \frac{5 d-3 c+5 b}{d+b-3 c}$. This implies that if $(5 d-3 c+5 b)(d+b-3 c)>0$ there are four axes $X= \pm \sqrt{\nu_{ \pm}} Y$ which correspond to bifurcated submaximal solutions in 
Fix $\left(\kappa^{\prime \prime}\right)$. And if $(5 d-3 c+5 b)(d+b-3 c)<0$, no submaximal solution can bifurcate in this plane.

- If $b+d=c$ and $c \neq 0$, then $K(X, Y)=-2 c\left(X^{2}+E_{3}^{2} Y^{2}\right)^{4}$. There is no bifurcated submaximal solution in $\operatorname{Fix}\left(\kappa^{\prime \prime}\right)$.

- Suppose that $d+b=0$ and $c \neq 0$, then $K(X, Y)=-3 c\left(X^{2}-E_{3}^{2} Y^{2}\right)^{2}$ and the axes $X= \pm E_{3} Y$ correspond to bifurcated submaximal solutions in $\operatorname{Fix}\left(\kappa^{\prime \prime}\right)$.

- Finally, if $(b+d)(b+d-c)<0$, then $K$ has no other root than $(0,0)$. By the same argument as before, this shows that no submaximal solution can bifurcate in fixed-point plane Fix $\left(\kappa^{\prime \prime}\right)$.

Stability of the solutions. The transverse eigenvalues for isotropy type $\widetilde{C}_{2 \kappa}$ and $\widetilde{C}_{2 \kappa}^{\prime}$ are summarized in Table 9.

\begin{tabular}{|c|c|c|}
\hline Isotropy type & $\widetilde{C}_{2 \kappa}$ & $\widetilde{C}_{2 \kappa}^{\prime}$ \\
\hline Tranvserse eigenvalue & $8(3+2 \sqrt{2})(d+b-3 c) X^{4}$ & $-8(3-2 \sqrt{2})(d+b-3 c) X^{4}$ \\
\hline
\end{tabular}

Table 9: Transverse eigenvalues (leading order) of equilibria with maximal isotropy in $\operatorname{Fix}\left(\kappa^{\prime \prime}\right)$.

The bifurcation analysis of submaximal solutions is the same as in fixed-point plane Fix $(\sigma)$ and presents no difficulty.

Remark 4. From Tables 7, 8 and 9, we deduce that there always exists a range of parameters such that equilibria with isotropy type $\widetilde{C}_{2 \kappa}$ and $\widetilde{C}_{2 \kappa}^{\prime}$ are unstable. We also point out that, to leading order, the sign of transverse eigenvalues for isotropy type $\widetilde{C}_{2 \kappa}$ is the opposite of the sign of transverse eigenvalues for isotropy type $\widetilde{C}_{2 \kappa}^{\prime}$.

We can choose coordinates to express fixed-point lines Fix $\left(\widetilde{C}_{2 \kappa}\right)$ and Fix $\left(\widetilde{C}_{2 \kappa}^{\prime}\right)$ in $\mathbb{R}^{4}$ as Fix $\left(\widetilde{C}_{2 \kappa}\right)=$ $\{(x, x,-(1+\sqrt{2}) x,(1+\sqrt{2}) x) \mid x \in \mathbb{R}\}$ and Fix $\left(\widetilde{C}_{2 \kappa}^{\prime}\right)=\{(x,-x,(1+\sqrt{2}) x,(1+\sqrt{2}) x) \mid x \in \mathbb{R}\} . W e$ summerize in Table 10, to leading order, radial and transverse eigenvalues (denoted $t_{k}, k=1 \ldots 3$ ) of bifurcated branches $\widetilde{C}_{2 \kappa}$ and $\widetilde{C}_{2 \kappa}^{\prime}$ in $\mathbb{R}^{4}$.

\begin{tabular}{|c|c|c|}
\hline Isotropy type & $\widetilde{C}_{2 \kappa}$ & $\widetilde{C}_{2 \kappa}^{\prime}$ \\
\hline Radial eigenvalue & $8(2+\sqrt{2}) A x^{2}$ & $8(2+2 \sqrt{2}) A x^{2}$ \\
\hline$t_{1}$ & $128(3+2 \sqrt{2})(b-d) x^{4}$ & $-128(3+2 \sqrt{2})(b-d) x^{4}$ \\
\hline$t_{2}$ & $-32(3+2 \sqrt{2})(b+c+9 d) x^{4}$ & $32(3+2 \sqrt{2})(b+c+9 d) x^{4}$ \\
\hline$t_{3}$ & $32(3+2 \sqrt{2})(b+d-3 c) x^{4}$ & $-32(3+2 \sqrt{2})(b+d-3 c) x^{4}$ \\
\hline
\end{tabular}

Table 10: Radial and transverse eigenvalues (leading order) of bifurcated branches in $\mathbb{R}^{4}$. 


\section{Bifurcation of a heteroclinic network in the $\chi_{11}$ case}

\subsection{Existence}

We suppose now that the cubic term coefficient $A<0$, and by a suitable chage of time scale we can take $A=-1$. This implies, as shown in Theorem 5 , that a flow-invariant $S^{3}$ sphere bifurcates for Equations (12) and (13). The system reads:

$$
\left\{\begin{aligned}
\dot{z}_{1}= & \lambda z_{1}-z_{1}\left(\left|z_{1}\right|^{2}+\left|z_{2}\right|^{2}\right)+a z_{1}\left(\left|z_{1}\right|^{2}+\left|z_{2}\right|^{2}\right)^{2}+b\left(z_{1}^{4} \bar{z}_{2}+4 z_{2}^{3}\left|z_{1}\right|^{2}-z_{2}^{3}\left|z_{2}\right|^{2}\right) \\
& +c\left(3 \bar{z}_{1}^{2} z_{2}\left|z_{2}\right|^{2}-z_{1}^{2} \bar{z}_{2}^{3}-2 \bar{z}_{1}^{2}\left|z_{1}\right|^{2} z_{2}\right)+d\left(-5 \bar{z}_{1}^{4} \bar{z}_{2}+\bar{z}_{2}^{5}\right)+\text { h.o.t. } \\
\dot{z}_{2}= & \lambda z_{2}-z_{2}\left(\left|z_{1}\right|^{2}+\left|z_{2}\right|^{2}\right)+a z_{2}\left(\left|z_{1}\right|^{2}+\left|z_{2}\right|^{2}\right)^{2}+b\left(-\bar{z}_{1} z_{2}^{4}-4 z_{1}^{3}\left|z_{2}\right|^{2}+z_{1}^{3}\left|z_{1}\right|^{2}\right) \\
& +c\left(-3 z_{1} \bar{z}_{2}^{2}\left|z_{1}\right|^{2}+\bar{z}_{1}^{3} z_{2}^{2}+2 z_{1} \bar{z}_{2}^{2}\left|z_{2}\right|^{2}\right)+d\left(5 \bar{z}_{1} \bar{z}_{2}^{4}-\bar{z}_{1}^{5}\right)+\text { h.o.t. }
\end{aligned}\right.
$$

In the sequel we also suppose that coefficients $(b, c, d)$ satisfy the following conditions:

- $\mathbf{C 1}: b-d>0$

- C2: $d(3 d-b-c)<0$ and $b+c+9 d>0$

- C3: $(b+d)(b+d-c)<0$ and $b+d-3 c<0$

Under these conditions all bifurcated equilibria have maximal isotropy and moreover, according to Remark 4, none of them is stable. More precisely, condition C1 implies that saddle-sink heteroclinic orbits connect in the plane $\operatorname{Fix}(\kappa)$ equilbria of isotropy type $\widetilde{C}_{2 \kappa}$ to equilibria with isotropy type $\widetilde{C}_{2 \kappa}^{\prime}$. Condition C2 implies that saddle-sink heteroclinic orbits connect in the plane $\operatorname{Fix}(\sigma)$ equilibria with isotropy type $\widetilde{C}_{2 \kappa}^{\prime}$ to equilibria with isotropy type $\widetilde{C}_{2 \kappa}$ (case $I$ in Figure 10$)$. In the same fashion, saddle-sink heteroclinic orbits connect in the plane $\operatorname{Fix}\left(\kappa^{\prime \prime}\right)$ equilibria with isotropy type $\widetilde{C}_{2 \kappa}$ to eqiulibria with isotropy type $\widetilde{C}_{2 \kappa}^{\prime}$ when condition $\mathbf{C 3}$ is satisfied.

These heteroclinic orbits are robust against $\mathcal{G}$-equivariant perturbations. Their $\mathcal{G}$-orbit realizes a heteroclinic network between the $\mathcal{G}$-orbits of equilibria of types $\widetilde{C}_{2 \kappa}$ and $\widetilde{C}_{2 \kappa}^{\prime}$.

Notice that under the above hypotheses the equilibria of type $\widetilde{C}_{2 \kappa}$ have a one dimensional unstable manifold, while equilibria of type $\widetilde{C}_{2 \kappa}^{\prime}$ have a two dimensional unstable manifold which contains the heteroclinic orbits lying in the planes of type $\operatorname{Fix}(\sigma)$ and $\operatorname{Fix}\left(\kappa^{\prime \prime}\right)$.

The existence of a heteroclinic network can lead to interesting non trivial dynamics characterized by long periods of quasi-static state (trajectory approaches an equilibrium of the cycle) followed by a fast excursion far from equilibrium and relaxation to another quasi-static state, the process being repeated in an aperiodic way $[11,2,24]$. This point will be considered in Section 5.3 , but we first simplify the problem by proceeding to a suitable orbit space reduction. 


\subsection{Quotient network}

The heteroclinic network introduced above has 48 nodes (equilibria) and 144 edges (heteroclinic orbits). Indeed the isotropy subgroups $\widetilde{C}_{2 \kappa}$ and $\widetilde{C}_{2 \kappa}^{\prime}$ have order 4 , hence the orbits of equilibria with these isotropies have $|\mathcal{G}| / 4=24$ elements each. To each node of type $\widetilde{C}_{2 \kappa}$ are associated 2 "outgoing" edges and 4 "incoming" edges. There are 48 nodes but each edge has two ends, hence the result. We can simplify this structure by projecting the system onto the quotient space (orbit space) $S^{3} / \mathcal{G}$ where $S^{3}$ is the flow-invariant sphere. This procedure would project the network onto a simpler one in which there are only two nodes. Moreover the trajectories of the equivariant vector field in $S^{3}$ project on trajectories for a smooth vector field defined on the orbit space [11]. However this orbit space is not a manifold (it would be if the action of $\mathcal{G}$ were free) and its geometric, stratified structure is too difficult to compute to make this method useful in our case. We can however proceed as [1] by identifying a subgroup $G_{0}$ of $\mathcal{G}$ with a free action on $S^{3}$ and large enough to allow for a substantial reduction of the number of equilibria on the 3 -dimenisonal manifold $S^{3} / G_{0}$. This is the aim of the next lemma.

Lemma 11. The group $G_{0}$ generated by the elements $\rho^{2}$ and $\epsilon$ has 24 elements. It acts fixed-point free on $S^{3}$ and the two $\mathcal{G}$-orbits of equilibria on $S^{3}$ reduce to a pair of equilibria in the manifold $S_{3} / G_{0}$ for the projected dynamics.

Proof. In Table 4 of [14], $G_{0}$ is identified with the 24 element group $S L(2,3)$, the group of $2 \times 2$ matrices over the field $\mathbb{Z}_{3}$. Since none of its elements appears in the isotropy subgroups of $\mathcal{G}$ for the representation $\chi_{11}$, its elements only fix the origin. For the same reason $G_{0}$ acts fixed point free on the 24 elements orbits of equilibria and by taking the quotient by this action these orbits reduce to single equilibria.

It follows that the heteroclinic network "drops down" to a quotient heteroclinic network between the two equilibria which we denote by $A\left(\widetilde{C}_{2 \kappa}\right.$ type) and $B\left(\widetilde{C}_{2 \kappa}^{\prime}\right.$ type $)$ in $S^{3} / G_{0}$. There are two connections from $A$ to $B$ and four connections from $B$ to $A$, as it can be seen in Figure 12. This projected heteroclinic network can be seen as the union of eight heteroclinic cycles which however belong to two symmetry classes only: the cycles $1 \rightarrow 5,2 \rightarrow 5,1 \rightarrow 6,2 \rightarrow 6$ are exchanged by reflection symmetries (projected on $S^{3} / G_{0}$ ), same thing for the cycles $3 \rightarrow 5,4 \rightarrow 5,3 \rightarrow 6,4 \rightarrow 6$. We call $\nu$-cycle (resp. $\mu$-cycle) the cycle $1 \rightarrow 5$ (resp. $4 \rightarrow 5$ ). We denote $\nu_{A}, \nu_{B}$ (resp. $\left.\mu_{A}, \mu_{B}\right)$ the eigenvalues at $A$ and $B$ along the connection $1-2($ resp. $3-4)$.

\subsection{Asymptotic stability}

The asymptotic stability of heteroclinic cycles has been studied by several authors [33, 34, 3, 35] and sufficient conditions on the ratio of eigenvalues "along" the cycle have been provided to ensure this 


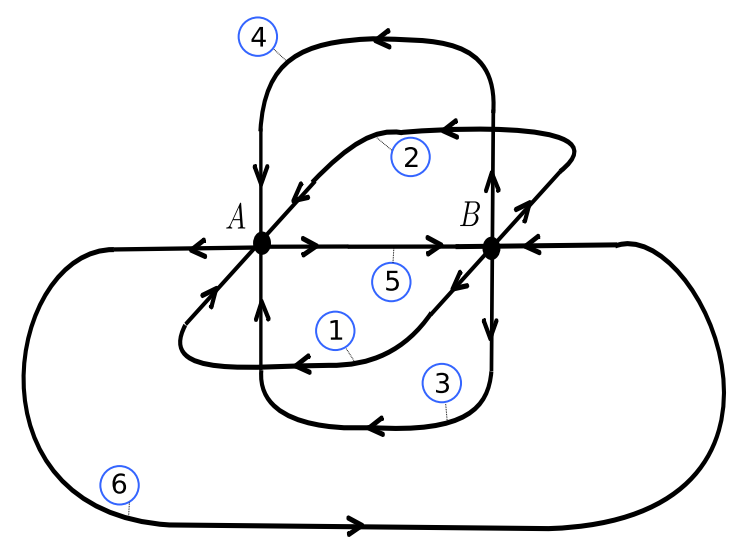

Figure 12: Representation of the quotient heteroclinic network between equilibria $A\left(\widetilde{C}_{2 \kappa}\right.$ type) and $B$ $\left(\widetilde{C}_{2 \kappa}^{\prime}\right.$ type) in $S^{3} / G_{0}$. Heteroclinic connections denoted 1,2 , which link $B$ to $A$, result of the quotient in $S^{3} / G_{0}$ of the heteroclinic connections which connect $\widetilde{C}_{2 \kappa}^{\prime \prime}$ to $\widetilde{C}_{2 \kappa}$ in $\operatorname{Fix}(\sigma)$. Heteroclinic connections denoted 3,4 , which link $B$ to $A$, result of the quotient in $S^{3} / G_{0}$ of the heteroclinic connections which connect $\widehat{C}_{2 \kappa}$ to $\widetilde{C}_{2 \kappa}^{\prime}$ in $\operatorname{Fix}\left(\kappa^{\prime \prime}\right)$. Heteroclinic connections denoted 5,6 , which link $A$ to $B$, result of the quotient in $S^{3} / G_{0}$ of the heteroclinic connections which connect $\widetilde{C}_{2 \kappa}$ to $\widetilde{C}_{2 \kappa}^{\prime}$ in $\operatorname{Fix}(\kappa)$.

property generically. Roughly speaking, the attractiviness property of a heteroclinic cycle is determined by the relative strength of the contracting and expanding eigenvalues along the cycle, computed at the equilibria in the cycle. If at an equilibrium in the cycle the unstable manifold has dimension $>1$ and does not realize a saddle-sink connection to other equilibria in some fixed-point subspace, the heteroclinic cycle can not be asymptotically stable in the usual sense, that is asymptotically attracting for initial conditions in an open tubular neighborhood of the cycle. As shown by Krupa and Melbourne in [33], it can still have a weaker attractiviness property which they called essential stability: under certain conditions on the eigenvalues the heteroclinic cycle is attracting for initial conditions belonging to the complement of a cuspidal region in a tubular neighborhood of the cycle.

A heteroclinic network is a union of cycles. As observed by Kirk and Silber [31] those cycles can not be simultaneously essentially stable but conditions can be derived to determine which one is. In this section we derive sufficient conditions for the essential stability of the two cycles in our heteroclinic network projected on the orbit space $S^{3} / G_{0}$.

First we simplify notation by denoting $\left(\lambda_{A},-\nu_{A},-\mu_{A}\right)$ the eigenvalues at equilibrium $A$ and $\left(-\lambda_{B}, \nu_{B}, \mu_{B}\right)$ the eigenvalues at equilibrium $B$. We consider:

$$
\lambda_{e}>0, \quad \nu_{e}>0, \quad \mu_{e}>0 \quad e=A, B
$$

The hypotheses of [33] do not apply but we will proceed in the same fashion as in [31]. In the following, 
we suppose without loss of generality that:

$$
\nu_{B}>\mu_{B}
$$

We define:

$$
\rho_{\mu}=\frac{\mu_{A} \lambda_{B}}{\lambda_{A} \mu_{B}}, \quad \sigma_{\mu}=\frac{\mu_{A}}{\lambda_{A}}\left[\frac{\nu_{B}}{\mu_{B}}-\frac{\nu_{A}}{\mu_{A}}\right], \quad \rho_{\nu}=\frac{\nu_{A} \lambda_{B}}{\lambda_{A} \nu_{B}}, \quad \sigma_{\nu}=\frac{\nu_{A}}{\lambda_{A}}\left[\frac{\mu_{B}}{\nu_{B}}-\frac{\mu_{A}}{\nu_{A}}\right]
$$

Theorem 6. (i) Suppose that $\rho_{\mu}>1$ and $\rho_{\nu}>1$.

1. If $\sigma_{\nu}<0$ and $\sigma_{\mu}>0$, almost all orbits passing through a tubular neighborhood of the $\mu$-cycle escape this neighborhood in finite time, exceptions being those orbits that lie in the stable manifolds of $A$ or $B$. The $\nu$-cycle is essentially asymptotically stable: it attracts almost all trajectories starting in a small enough tubular neighborhood of it, the only possible exceptions being those orbits that pass through a cuspoidal region abutting the heteroclinic connection from $A$ to $B$.

2. If $\sigma_{\nu}>0$ and $\sigma_{\mu}<0$, almost all orbits passing through a tubular neighborhood of the $\nu$-cycle escape this neighborhood in finite time, exceptions being those orbits that lie in the stable manifolds of $A$ or $B$. The $\mu$-cycle is essentially asymptotically stable: it attracts almost all trajectories starting in a small enough tubular neighborhood of it, the only possible exceptions being those orbits that pass through a cuspoidal region abutting the heteroclinic connection from $A$ to $B$.

(ii) Suppose that $0<\rho_{\mu}<1$ (resp. $0<\rho_{\nu}<1$ ). Then the $\mu$-cycle (resp. $\nu$-cycle) repels almost all orbits and the attractivity properties of the $\nu$-cycle (esp. $\mu$-cycle) are determined by $\sigma_{\nu}\left(\right.$ resp. $\left.\sigma_{\mu}\right)$ as above.

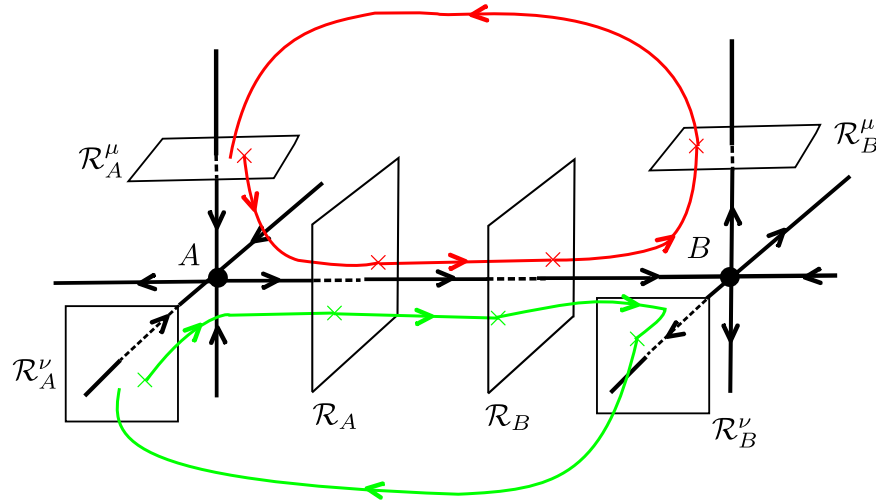

Figure 13: First return map in $S^{3}$. 
Proof. We apply the method for stability analysis of heteroclinic cycles as exposed in [33] and [34], to which we refer for justifications. We first linearize the flow in neighborhoods of $A$ and $B$ by local $C^{1}$ changes of variables. This requires that a finite set of nonresonance conditions between the eigenvalues at $A$ and $B$ are satisfied. Such conditions are generic and can be verified numerically in our case. In fact they can also be removed as shown for example in another context in [21]. We can further choose the local coordinates such that the local stable and unstable manifolds of $A$ and $B$ are either the horizontal axis or the vertical plane. Using Euclidean coordinates $(v, w)$ in the vertical plane and $u$ for the horizontal axis, we have for $A$ :

$$
W_{\text {loc }}^{u}(A)=\{(u, 0,0) \mid u \in \mathbb{R}\} \quad W_{\text {loc }}^{s}(A)=\left\{(0, v, w) \mid(v, w) \in \mathbb{R}^{2}\right\}
$$

and for $B$ :

$$
W_{\text {loc }}^{s}(B)=\{(u, 0,0) \mid u \in \mathbb{R}\} \quad W_{\text {loc }}^{u}(B)=\left\{(0, v, w) \mid(v, w) \in \mathbb{R}^{2}\right\}
$$

The linearized vector field about $A$ is

$$
\begin{aligned}
& \dot{u}=\lambda_{A} u \\
& \dot{v}=-\nu_{A} v \\
& \dot{w}=-\mu_{A} w
\end{aligned}
$$

and about $B$ :

$$
\begin{aligned}
& \dot{u}=-\lambda_{B} u \\
& \dot{v}=\nu_{B} v \\
& \dot{w}=\mu_{B} w
\end{aligned}
$$

We now define rectangular cross sections in neighborhoods of $e, e=A, B$ (see Figure 13):

$$
\begin{aligned}
& \mathcal{R}_{e}=\left\{(u, v, w) \mid u=1, \quad-v_{e} \leq v \leq v_{e}, \quad-w_{e} \leq w \leq w_{e}\right\} \\
& \mathcal{R}_{e}^{\mu}=\left\{(u, v, w) \mid w=1, \quad-u_{e} \leq u \leq u_{e}, \quad-v_{e} \leq v \leq v_{e}\right\} \\
& \mathcal{R}_{e}^{\nu}=\left\{(u, v, w) \mid v=1, \quad-u_{e} \leq u \leq u_{e}, \quad-w_{e} \leq w \leq w_{e}\right\}
\end{aligned}
$$

We can then build two first return maps $\Psi^{\mu}: \mathcal{R}_{A}^{\mu} \rightarrow \mathcal{R}_{A}^{\mu}$ and $\Psi^{\nu}: \mathcal{R}_{A}^{\nu} \rightarrow \mathcal{R}_{A}^{\nu}$ as follows: $\Psi^{\mu}=\Psi_{B A}^{\mu} \circ \Phi_{B}^{\mu} \circ \Psi_{A B} \circ \Phi_{A}^{\mu}$ and $\Psi^{\nu}=\Psi_{B A}^{\nu} \circ \Phi_{B}^{\nu} \circ \Psi_{A B} \circ \Phi_{A}^{\nu}$ where

$$
\begin{gathered}
\Phi_{A}^{\mu}: \mathcal{R}_{A}^{\mu} \rightarrow \mathcal{R}_{A} \quad \Phi_{B}^{\mu}: \mathcal{R}_{B} \rightarrow \mathcal{R}_{B}^{\mu} \quad \Phi_{A}^{\nu}: \mathcal{R}_{A}^{\nu} \rightarrow \mathcal{R}_{A} \quad \Phi_{B}^{\nu}: \mathcal{R}_{B} \rightarrow \mathcal{R}_{B}^{\nu} \\
\Psi_{A B}: \mathcal{R}_{A} \rightarrow \mathcal{R}_{B} \quad \Psi_{B A}^{\mu}: \mathcal{R}_{B}^{\mu} \rightarrow \mathcal{R}_{A}^{\mu} \quad \Psi_{B A}^{\nu}: \mathcal{R}_{B}^{\nu} \rightarrow \mathcal{R}_{A}^{\nu}
\end{gathered}
$$


The local maps $\Phi_{A}^{\mu}$ and $\Phi_{A}^{\nu}$ are obtained by integrating the equations for the flow linearized about A:

$$
\begin{gathered}
\Phi_{A}^{\mu}(u, v, 1)=\left(1, v u^{\frac{\nu_{A}}{\lambda_{A}}}, u^{\frac{\mu_{A}}{\lambda_{A}}}\right) \text { with } u \neq 0 \\
\Phi_{A}^{\nu}(u, 1, w)=\left(1, u^{\frac{\nu_{A}}{\lambda_{A}}}, w u^{\frac{\mu_{A}}{\lambda_{A}}}\right) \text { with } u \neq 0
\end{gathered}
$$

Same thing for the maps $\Phi_{B}^{\mu}$ and $\Phi_{B}^{\nu}$ :

$$
\begin{aligned}
& \Phi_{B}^{\mu}(1, v, w)=\left(w^{\frac{\lambda_{B}}{\mu_{B}}}, v w^{-\frac{\nu_{B}}{\mu_{B}}}, 1\right) \text { with } w^{\frac{\nu_{B}}{\mu_{B}}}>v \geq 0 \\
& \Phi_{B}^{\nu}(1, v, w)=\left(v^{\frac{\lambda_{B}}{\nu_{B}}}, 1, w v^{-\frac{\mu_{B}}{\nu_{B}}}\right) \text { with } v>w^{\frac{\nu_{B}}{\mu_{B}}} \geq 0
\end{aligned}
$$

where $\mathcal{C}_{B}^{\mu}=\left\{(v, w) \in \mathcal{R}_{B} \mid w^{\frac{\nu_{B}}{\mu_{B}}}>v \geq 0\right\}$ and $\mathcal{C}_{B}^{\nu}=\left\{(v, w) \in \mathcal{R}_{B} \mid v>w^{\frac{\nu_{B}}{\mu_{B}}} \geq 0\right\}$ are complementary domains in $\mathcal{R}_{B}$ of the maps $\Phi_{B}^{\mu}$ and $\Phi_{B}^{\nu}$. Note that the point at which a trajectory intersects $\mathcal{R}_{B}$ determines whether the trajectory leaves the vacinity of $B$ in the direction of $A$ through $\mathcal{R}_{B}^{\mu}$ or $\mathcal{R}_{B}^{\nu}$. Condition (15) implies that $\mathcal{C}_{B}^{\mu}$ is a cuspoidal region of $\mathcal{R}_{B}^{\mu}$.

By exploiting the equivariance of the vector field, we obtain for the "global" maps $\Psi_{A B}, \Psi_{B A}^{\mu}$ : and $\Psi_{B A}^{\nu}$ :

$$
\begin{aligned}
& \Psi_{A B}(1, v, w)=\left(1, \alpha_{A B} v, \beta_{A B} w\right)+\text { h.o.t } \\
& \Psi_{B A}^{\mu}(u, v, 1)=\left(\alpha_{B A}^{\mu} u, \beta_{B A}^{\mu} v, 1\right)+\text { h.o.t } \\
& \Psi_{B A}^{\nu}(u, 1, w)=\left(\alpha_{B A}^{\nu} u, 1, \beta_{B A}^{\nu} w\right)+\text { h.o.t }
\end{aligned}
$$

where the $\alpha$ 's and $\beta$ 's are real coefficients.

- Study of the $\mu$-cycle. We consider trajectories that pass through $\mathcal{R}_{A}^{\mu}$ and then travel through a tubular neighborhood of the $\mu$-cycle before returning to $\mathcal{R}_{A}^{\mu}$. The behaviour of these trajectories is modelled by the return map $\Psi^{\mu}$ and we find to leading order:

$$
\Psi^{\mu}(u, v, 1)=\left(c_{1} u^{\rho_{\mu}}, c_{2} v u^{-\sigma_{\mu}}, 1\right) \text { with } 0 \leq v<c_{3} u^{\sigma_{\mu}}
$$

The domain of the return map is then defined as $\mathcal{D}_{A}^{\mu}=\left\{(u, v) \in \mathcal{R}_{A}^{\mu} \mid 0 \leq v<c_{3} u^{\sigma_{\mu}}\right\}$. A sufficient condition for $\mathcal{D}_{A}^{\mu}$ to be mapped into itself is $\sigma_{\mu}<0$. This follows from the observation that the image under $\Psi^{\mu}$ of the bounding surface defined by the equation $v=c_{3} u^{\sigma_{\mu}}$ is the boundary defined by $U=c_{4}$, where $c_{4}>0$ is some constant. Finally, if $\rho_{\mu}>1$ and $\sigma_{\mu}<0$ then $\Psi^{\mu}$ is a contraction on $\mathcal{D}_{A}^{\mu}$.

- Study of the $\nu$-cycle. We consider trajectories that pass through $\mathcal{R}_{A}^{\nu}$ and then travelonce through 
a tubular neighborhood of the $\nu$-cycle before returning to $\mathcal{R}_{A}^{\nu}$. The behaviour of these trajectories is modelled by the return map $\Psi^{\nu}$ and we find to leading order:

$$
\Psi^{\nu}(u, 1, w)=\left(c_{4} u^{\rho_{\nu}}, 1, c_{5} w u^{-\sigma_{\nu}}, 1\right) \text { with } 0 \leq w<c_{6} u^{\sigma_{\nu}}
$$

The study is analogous to that for the $\mu$-cycle. If $\rho_{\nu}>1$ and $\sigma_{\nu}<0$ then the $\nu$-cycle attracts all trajectories that cross $\mathcal{R}_{A}^{\nu}$ sufficiently close to the origin.

The main difference between the results obtained for the $\mu$-cycle and the $\nu$-cycle comes from the condition (15). If $\rho_{\nu}>1$ and $\sigma_{\nu}<0$ the $\nu$-cycle attracts almost all trajectories that lie near the heteroclinic connection from $A$ to $B$, while, if $\rho_{\mu}>1$ and $\sigma_{\mu}<0$ the $\mu$-cycle attracts just trajectories in a cuspoidal region emananting from the heteroclinic connection.

It is not possible that both $\sigma_{\mu}$ and $\sigma_{\nu}$ be simultaneously positive:

$$
\sigma_{\mu}=-\frac{\nu_{B}}{\mu_{B}} \sigma_{\nu}
$$

Note that if $\sigma_{\mu}>0$ when $\rho_{\nu}, \rho_{\mu}>1$, then almost all trajectories near the $\mu$-cycle eventually leave it in the direction of the $\nu$-cycle. However, since $\sigma_{\nu}<0$ in this case, the trajectories that switch to the $\nu$-cycle can not at a later time switch back to the $\mu$-cycle.

\subsection{Computation of the stability conditions}

In principle the stability conditions stated in Theorem 6 are easy to compute. In our case however there is a difficulty which comes from the fact that for the system (14), which is truncated at order 5 , the expanding and contracting eigenvalues along a given connection have exactly the same magnitude (see Table 10). It is interesting to observe that this follows from a property of reversibility of the vector field on the invariant sphere, as the next lemma shows (proof of the lemma is straightforward).

Lemma 12. Let $\mathbf{s}$ be the transformation in $\mathbb{R}^{4}$ defined by $\mathbf{s}\left(z_{1}, \bar{z}_{1}, z_{2}, \bar{z}_{2}\right)=\left(z_{2}, \bar{z}_{2}, z_{1}, \bar{z}_{1}\right)$. Let us rewrite $X=\left(z_{1}, \bar{z}_{1}, z_{2}, \bar{z}_{2}\right)$ and Equation (14) in the form

$$
\dot{X}=\left(\lambda-\|X\|^{2}+b\|X\|^{4}\right) X+E_{c, d}(X) \text { with } E_{c, d}(X)=c E_{5,3}(X)+d E_{5,4}(X)
$$

Then $E_{c, d}(\mathbf{s} X)=-\mathbf{s} E_{c, d}(X)$ for all $X$. Moreover, Fix $\left(\widetilde{C}_{2 \kappa}^{\prime}\right)=\mathbf{s} F i x\left(\widetilde{C}_{2 \kappa}\right)$.

Remark 5. We recall that $E_{5,3}$ (resp. $\left.E_{5,4}\right)$ is the quintic equivariant map in factor of $c$ (resp. d) in Equations (12) and (13) of Theorem 4, see Appendix C.2 for the computations. 
Now let's let $X=r U, U \in S^{3}$. The system (14) decouples in a radial part and tangential part:

$$
\begin{aligned}
\dot{r} & =\left(\lambda-r^{2}+b r^{4}\right) r+r^{5}\left\langle E_{c, d}(U), U\right\rangle \\
\dot{U} & =r^{4}\left[E_{c, d}(U)-\left\langle E_{c, d}(U), U\right\rangle U\right]=r^{4} H(U)
\end{aligned}
$$

By lemma 12 the tangential part is a reversible vector field. Let $X_{0}=r_{0} U_{0}$ be an equilibrium on $\operatorname{Fix}\left(\widetilde{C}_{2 \kappa}\right)$ and $X_{0}^{\prime}=\mathbf{s} X_{0}=r_{0} \mathbf{s} U_{0}$. Then $X_{0}^{\prime}$ is also an equilibrium, moreover $D H\left(\mathbf{s} U_{0}\right)=-\mathbf{s} D H\left(U_{0}\right) \mathbf{s}$, which implies that the transverse eigenvalues at $X_{0}^{\prime}$ are exactly opposite to the tranverse eigenvalues at $X_{0}$. This property is conserved by projection of the system on the orbit space $S^{3} / G_{0}$.

It is therefore necessary to consider the 7th order expansion of the system in order to remove this degeneracy. There are 12 equivariant terms of order 7 (see Appendix B). We have checked that some of these terms are not reversible, for example the following vector field which we note $E_{7}$ :

$$
\begin{aligned}
& \dot{z}_{1}=\bar{z}_{1}^{7}+7 \bar{z}_{1}^{3} \bar{z}_{2}^{4} \\
& \dot{z}_{2}=\bar{z}_{2}^{7}+7 \bar{z}_{2}^{3} \bar{z}_{1}^{4}
\end{aligned}
$$

Numerical simulations have been carried out with Matlab by introducing the term $E_{7}$ in the system:

$$
\dot{X}=\left(\lambda-\|X\|^{2}+b\|X\|^{4}\right) X+E_{c, d}(X)+e E_{7}(X)
$$

We give in Table 11, to leading order, the transverse eigenvalues of bifurcated branches in $\mathbb{R}^{4}$ depending upon the parameter $e$ of Equation 19. These transverse eigenvalues allow us to compute the stability conditions of Theorem 6. For the numerical simulations, the coefficient values are $\lambda=0.1$, $a=0, b=0.6, c=1.2 d=0.55$. We set $e=-1$ and we obtain:

$$
\begin{array}{lll}
\lambda_{A}=0.0011 & \nu_{A}=0.0745 & \mu_{A}=0.0266 \\
\lambda_{B}=0.0023 & \nu_{B}=0.0585 & \mu_{B}=0.0215
\end{array}
$$

which implies that:

$$
\rho_{\mu}=2.6336>1 \quad \sigma_{\mu}=-1.8221<0 \quad \rho_{\nu}=2.7060>1 \quad \sigma_{\nu}=0.6686>0
$$

Then we are in the second case of Theorem 6. Figure 14 shows one hour runs with an initial condition close to an equilibrium with isotropy $\widetilde{C}_{2 \kappa}$. For the value $e=-1$ the solution converges to a heteroclinic 
cycle of type $\mu$-cycle, while for $e=3$ none of the heteroclinic cycles are stable.

\begin{tabular}{|c|c|c|}
\hline Isotropy type & $\widetilde{C}_{2 \kappa}$ & $\widetilde{C}_{2 \kappa}^{\prime}$ \\
\hline$t_{1}(e)$ & $128(3+2 \sqrt{2})\left(b-d+(2+\sqrt{2}) e x^{2}\right) x^{4}$ & $-128(3+2 \sqrt{2})\left(b-d-(2+\sqrt{2}) e x^{2}\right) x^{4}$ \\
\hline$t_{2}(e)$ & $-32(3+2 \sqrt{2})\left(b+c+9 d+24(2+\sqrt{2}) e x^{2}\right) x^{4}$ & $32(3+2 \sqrt{2})\left(b+c+9 d-24(2+\sqrt{2}) e x^{2}\right) x^{4}$ \\
\hline$t_{3}(e)$ & $32(3+2 \sqrt{2})\left(b+d-3 c-10(2+\sqrt{2}) e x^{2}\right) x^{4}$ & $-32(3+2 \sqrt{2})\left(b+d-3 c+10(2+\sqrt{2}) e x^{2}\right) x^{4}$ \\
\hline
\end{tabular}

Table 11: Transverse eigenvalues (leading order) of bifurcated branches in $\mathbb{R}^{4}$ depending upon the parameter $e$ of Equation 19.

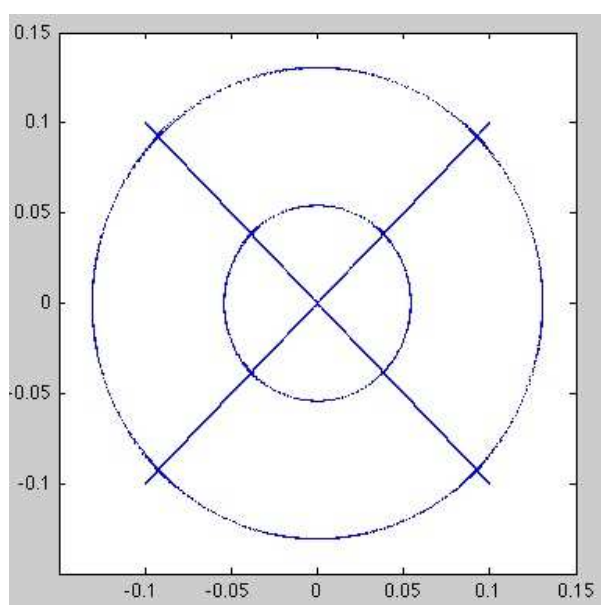

(a) $e=-1$.

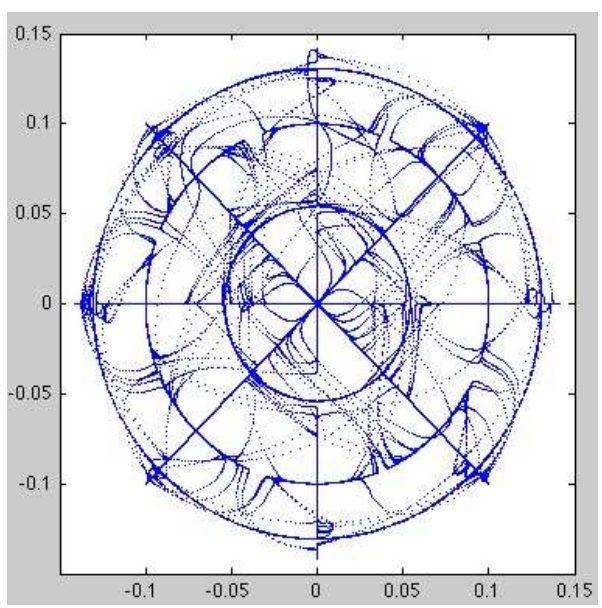

(b) $e=3$.

Figure 14: Projection on the plane $\left(x_{1}, y_{1}\right)$ of a trajectory of (19) with initial condition near an equilibrium of type $\widetilde{C}_{2 \kappa}$. Coefficient values in both cases are $\lambda=0.1, a=0, b=0.6, c=1.2 d=0.55$.

\section{Conclusion}

In this paper we have completed the bifurcation analysis of periodic patterns, introduced in [14], for neural field equations defined on the Poincaré disc $\mathcal{D}$. These equations are assumed invariant under the action of the lattice group $\Gamma$ of $\mathrm{U}(1,1)$ whose fundamental domain is the regular octagon. We have computed the bifurcation diagrams for the three irreducible representations of dimension four, henceforth completing the classification started in [14], in which bifurcation diagrams for the representations of dimension less than four were obtained and shown to correspond to well known goup actions. We have proved that for two of the four-dimensional irreducible representations, generically, there always exist stable equilibria with a given isotropy type. For the third representation we have presented bifurcation diagrams in fixedpoint planes and also shown that: (i) bifurcation of submaximal solutions can be generic, (ii) bifurcation of a heteroclinic network connecting the equilibria with maximal isotropy type can also occur generically. In the final section, a stability analysis of this heteroclinic network was presented. 
The existence of the heteroclinic network raises many interesting questions from the neuroscience point of view. Metastability in neuronal network has been observed in the brains of anaesthetized animals where the cortex seems to show an intrinsic pattern of activity that evolves over time by switching among a specific set of states [30, 40, 22]. It has also been shown that metastable states play a key role in the execution of cognitive functions. Indeed, experimental and modeling studies suggest that most of these functions are the result of transient activity of large-scale brain networks in the presence of noise $[38,39]$. In our case the spontaneous activity represented by the heteroclinic network corresponds to switches between multiple states where each state is a specific textural feature. Despite the fact that it seems unrealistic to investigate experimentally the predicted behaviour, the presence of the heteroclinic network is nonetheless an interesting mechanism which sould be taken into account for the validation of our texture model.

The nonlinear integro-differential equation studied in this paper models one hypercolmun of the visual area $\mathrm{V} 1$ and is therefore local: the average action potential $V$ does not depend of the position in the visual cortex. Another exciting question comes from the direct spatialization of our model. The primary visual cortex can be partitioned into hypercolumns on a lattice describing the distribution of singularities or pinwheels in the orientation preference map. Bressloff and Cowan have introduced several models which take account of this functional architecture of the cortex. Their approach has led to an elegant interpretation for the occurence of geometric hallucinations [10]. An interesting avenue for future work is to study the spatio-temporal patterns produced by the spatialized model, where the average membrane potential now depends on the location in the visual cortex in addition to the structure tensor and time.

Our study raises several questions from the point of view of pattern formation in the Poincaré disk. As in the Euclidean case of pattern formation, we look for solutions in the restricted class of patterns which are spatially periodic, this means looking for bifurcating patterns which are invariant under the action of a discrete subgroup $\Gamma$ of $U(1,1)$ whose fundamental domain is a polygon. There are, however big differences with the Euclidean case. First of all, as explained in the introduction, tilings of the Poincaré disk exist with polygons having an arbitrary number of sides, while in $\mathbb{R}^{2}$ only rectangular, square and hexagonal periodic tilings exist. However, while in the Euclidean case any critical wave number can be associated with a periodic lattice, in the hyperbolic case this is not true. Indeed, the size of a regular polygon with a given number of vertices is fixed in hyperbolic geometry as a consequence of the Gauss-Bonnet formula (Katok [29]). We focused our work on the particular case of an octagon because of its relatively simple interpretation in term of retinal input [13], but non-octagonal tilings can also be studied. We think that the methods presented in [14] and in this paper can be applied to each lattice of the Poincaré disk. Our neural fields equation with a single population of neurons exhibits at the first bifurcation point only steady-state bifurcation. It could also be interesting to model two 
populations of neurons (excitatory/inhibitory for example) and look for Hopf-bifurcation.

\section{Acknowledgments}

The authors are glad to thank R. Lauterbach for providing a GAP program to determine the biquaternionic form of $\mathcal{G}$ as well as its four-dimensional irreducible representations. We also thank J. Rankin for his helpful comments on the preparation of the present manuscript. This work was partially funded by the ERC advanced grant NerVi number 227747.

\section{APPENDIX}

\section{A Presentation with biquaternions}

It is natural to identify the finite group $\mathcal{G}$ to a group of $4 \times 4$ real matrices as $\operatorname{dim}\left(\chi_{12}\right)=4$. Lauterbach and Matthews [36] have successfuly introduced biquaternions to study equivariant dynamical systems with $\mathbf{S O}(4)$ symmetry. Here we also use biquaterions to give a geometric way to describe the group $\mathcal{G}$. We denote by $\mathcal{Q}$ the set of unit quaternions. The set of pairs of such quaternions forms a group, called the spinor group and denoted by $\mathrm{Spin}_{4}$. We get a map [16]:

$$
\operatorname{Spin}_{4} \rightarrow \mathbf{S O}(4):(l, r) \mapsto[l, r]=\{x \mapsto \bar{l} x r\}
$$

where a vector $\mathbf{x} \in \mathbb{R}^{4}$ is identified with a quaternion $x \in \mathbb{H}$ via

$$
\mathbf{x}=\left(\begin{array}{l}
x_{1} \\
x_{2} \\
x_{3} \\
x_{4}
\end{array}\right) \Leftrightarrow x=e x_{1}+i x_{2}+j x_{3}+k x_{4}
$$

The two following propositions hold.

Proposition 2. For the irreductible representations $\chi_{12}, \chi_{13}$, the group $\mathcal{G}$ admits the following presentation with biquaternions:

$$
\mathcal{G}=\left\langle[j, e],\left[\frac{\sqrt{2}}{2}(j+k), j\right],[e, i],[i, e],\left[\frac{1}{2}(-e+i+j+k), \frac{1}{2}(\sqrt{3} e+i)\right]\right\rangle
$$


It is also possible to identify the generators of $\mathcal{G}$ in matrix form:

$$
\kappa=\left[\begin{array}{cccc}
0 & 0 & 0 & -1 \\
0 & 0 & -1 & 0 \\
0 & -1 & 0 & 0 \\
-1 & 0 & 0 & 0
\end{array}\right], \quad \rho=\frac{\sqrt{2}}{2}\left[\begin{array}{cccc}
0 & 0 & -1 & -1 \\
0 & 0 & 1 & -1 \\
-1 & -1 & 0 & 0 \\
1 & -1 & 0 & 0
\end{array}\right], \quad \sigma=\frac{\sqrt{2}}{4}\left[\begin{array}{cccc}
1 & \sqrt{3} & -1 & \sqrt{3} \\
\sqrt{3} & -1 & -\sqrt{3} & -1 \\
-1 & -\sqrt{3} & -1 & \sqrt{3} \\
\sqrt{3} & -1 & \sqrt{3} & 1
\end{array}\right]
$$

Proof. The computer algebra program GAP gives the presentation of $\mathcal{G}$ as $\mathcal{G}=\left\langle m_{1}, m_{2}, m_{3}, m_{4}, m_{5}\right\rangle$ with:

$$
m_{1}=[j, e], m_{2}=\left[\frac{\sqrt{2}}{2}(j+k), j\right], m_{3}=[e, i], m_{4}=[i, e], m_{5}=\left[\frac{1}{2}(-e+i+j+k), \frac{1}{2}(\sqrt{3} e+i)\right]
$$

We express each endomorphisms $\left(m_{l}\right)_{l=1 \ldots 5}$ of $\mathcal{G}$ in the canonical basis $\mathcal{B}=(e, i, j, k)$ and form the corresponding matrices $M_{l}=\mathcal{M a t}_{\mathcal{B}, \mathcal{B}}\left(m_{l}\right)$ for $l=1 \ldots 5$. A direct calculus shows that $\operatorname{trace}\left(M_{5}\right)=-\sqrt{3}$ and $M_{5}$ is of order 12 , such that we can write with our notations that (up to a conjugate) $M_{5}=-\epsilon \kappa$. Matrices $M_{1}, M_{3}, M_{4}$ are of order 4 and $M_{2}$ of order 2 . We set $\kappa=-M_{1} M_{3}$, such that $\epsilon=M_{5} M_{1} M_{3}$. We recognize that $M_{2}=\kappa^{\prime}=\rho \kappa$ then $\rho=-M_{2} M_{1} M_{3}$ and we verify that $\rho$ is of order 8. A straightforward calculus shows that $\rho^{2}=-M_{4}$ and we finally note $\sigma=\rho^{-1} \epsilon^{-1}$. The expression of the matrices of generators of $\mathcal{G}$ are given in Eq. (20).

Proposition 3. For the irreductible representation $\chi_{11}$, the group $\mathcal{G}$ admits the following presentation with biquaternions:

$$
\mathcal{G}=\left\langle\left[\frac{1}{2}(-e+i+j+k), e\right],[e, j],[e,-e],[i, e],\left[\frac{\sqrt{2}}{2}(j+k), i\right]\right\rangle
$$

It is also possible to identify the generators of $\mathcal{G}$ in matrix form:

$$
\kappa=\left[\begin{array}{cccc}
0 & 0 & 0 & -1 \\
0 & 0 & 1 & 0 \\
0 & 1 & 0 & 0 \\
-1 & 0 & 0 & 0
\end{array}\right], \quad \rho=\frac{\sqrt{2}}{2}\left[\begin{array}{cccc}
0 & 1 & 1 & 0 \\
-1 & 0 & 0 & -1 \\
1 & 0 & 0 & -1 \\
0 & -1 & 1 & 0
\end{array}\right], \quad \sigma=\frac{\sqrt{2}}{2}\left[\begin{array}{cccc}
0 & 0 & -1 & 1 \\
0 & 0 & 1 & 1 \\
-1 & 1 & 0 & 0 \\
1 & 1 & 0 & 0
\end{array}\right]
$$

Proof. The proof is exactely the same as the previous one. 


\section{B Molien series}

In [11] we find theorems which allow to compute the vector space dimensions of the space of equivariant and invariant polynomial maps for a group action of a given degree. We recall that the set of $\mathcal{G}$-equivariant polynomial maps forms a module $\mathcal{M}$ over the $\operatorname{ring} \mathcal{R}_{\mathcal{G}}$ of $\mathcal{G}$-invariant polynomial maps. We denote by $r_{d}=\operatorname{dim} \overrightarrow{\mathcal{P}}^{d}(\mathcal{G})$ the dimension of the polynomial equivariants of degree $d$.

Theorem 7 (Equivariant Molien's theorem). Consider the formal power series

$$
\Phi_{\mathcal{M}}^{\rho}(z)=\sum_{d=0}^{\infty} r_{d} z^{d}
$$

It has a representation

$$
\Phi_{\mathcal{M}}^{\rho}(z)=\int_{\mathcal{G}} \frac{\operatorname{Tr}(g)}{\operatorname{det}(\mathbf{1}-z \rho(g))} d g
$$

In our case, $\mathcal{G}$ is a finite group, and we can directly apply this theorem together with table 3 to find:

- for $\chi_{12}$ :

$$
\begin{aligned}
\Phi_{\mathcal{M}}^{\chi_{12}}(z)=\frac{1}{96}\left[\frac{4}{(1-z)^{4}}-\frac{4}{(1+z)^{4}}\right. & +\frac{8}{1-z-z^{3}+z^{4}}-\frac{8}{1+z+z^{3}+z^{4}} \\
& \left.+\frac{8 \sqrt{3}}{1-z \sqrt{3}+2 z^{2}-z^{3} \sqrt{3}+z^{4}}-\frac{8 \sqrt{3}}{1+z \sqrt{3}+2 z^{2}+z^{3} \sqrt{3}+z^{4}}\right]
\end{aligned}
$$

and $\Phi_{\mathcal{M}}^{\chi_{12}}(z)=z+2 z^{3}+5 z^{5}+10 z^{7}+O\left(z^{7}\right)$

- for $\chi_{11}$ :

$$
\Phi_{\mathcal{M}}^{\chi_{11}}(z)=\frac{1}{96}\left[\frac{4}{(1-z)^{4}}-\frac{4}{(1+z)^{4}}-\frac{16}{\left(1+z+z^{2}\right)^{2}}+\frac{16}{\left(1-z+z^{2}\right)^{2}}\right]
$$

and $\Phi_{\mathcal{M}}^{\chi 11}(z)=z+z^{3}+4 z^{5}+12 z^{7}+O\left(z^{7}\right)$.

An analog of the Equivariant Molien's theorem holds for invariant polynomial mapsa dn we denote $c_{d}=\operatorname{dim} \mathcal{R}^{d}$ the dimension of invariants polynomials of degree $d$.

Theorem 8 (Invariant Molien's theorem). Consider the formal power series

$$
P_{\mathcal{R}_{\mathcal{G}}}^{\rho}(z)=\sum_{d=0}^{\infty} c_{d} z^{d}
$$

It has a representation

$$
P_{\mathcal{R}_{\mathcal{G}}}^{\rho}(z)=\int_{\mathcal{G}} \frac{1}{\operatorname{det}(\mathbf{1}-z \rho(g))} d g
$$

Applying this theorem together with tables 1 and 2 yields 
- for $\chi_{12}$ :

$$
P_{\mathcal{R}_{\mathcal{G}}}^{\chi_{12}}(z)=z^{2}+2 z^{4}+3 z^{6}+O\left(z^{6}\right)
$$

- for $\chi_{11}$ :

$$
P_{\mathcal{R}_{\mathcal{G}}}^{\chi_{11}}(z)=z^{2}+z^{4}+3 z^{6}+O\left(z^{6}\right)
$$

These results are summerized in the following table:

\begin{tabular}{|c|c|c|c|c|c|}
\hline Character & e3 & i4 & e5 & i6 & e7 \\
\hline$\chi_{11}$ & 1 & 1 & 4 & 3 & 12 \\
\hline$\chi_{12}$ & 2 & 2 & 5 & 3 & 10 \\
\hline$\chi_{13}$ & 2 & 2 & 5 & 3 & 10 \\
\hline
\end{tabular}

Table 12: The information on the number of invariant/equivariant polynomial maps for the irreductible representations $\chi_{11}, \chi_{12}$ and $\chi_{13}$. Here $e$ stands for equivariant, $i$ for invariant and the number behind these letters for the degree of the polynomial map. The number in the table gives the dimension of the space of equivariant/invariant polynomial maps in the given degrees.

\section{Computation of low-order equivariants}

\section{C.1 Computational part of the proof of theorem 2}

Proposition 4. For the irreductible representation $\chi_{12}$, the two cubic equivariant maps are:

$$
E_{1}(\mathbf{z})=\mathbf{z}\left(\left|z_{1}\right|^{2}+\left|z_{2}\right|^{2}\right) \text { and } E_{2}(\mathbf{z})=\left[\begin{array}{c}
\sqrt{3}\left(3 z_{1}^{2}+\bar{z}_{2}^{2}\right) \bar{z}_{1}-\mathbf{i}\left(z_{2}^{2}+3 \bar{z}_{1}^{2}\right) z_{2} \\
\sqrt{3}\left(3 \bar{z}_{1}^{2}+z_{2}^{2}\right) z_{1}+\mathbf{i}\left(\bar{z}_{2}^{2}+3 z_{1}^{2}\right) \bar{z}_{2} \\
\sqrt{3}\left(3 z_{2}^{2}+\bar{z}_{1}^{2}\right) \bar{z}_{2}+\mathbf{i}\left(z_{1}^{2}+3 \bar{z}_{2}^{2}\right) z_{1} \\
\sqrt{3}\left(3 \bar{z}_{2}^{2}+z_{1}^{2}\right) z_{2}-\mathbf{i}\left(\bar{z}_{1}^{2}+3 z_{2}^{2}\right) \bar{z}_{1}
\end{array}\right]
$$

Proof. Let $E$ denote a homogeneous equivariant mapping. We want to deduce the restrictions placed on the form of $E$ by the symmetry group $\mathcal{G}$. We first choose appropriate coordinates. Thanks to proposition 2 of appendix A we have a presentation of $\mathcal{G}$ with $4 \times 4$ real matrices with generators $\rho, \sigma, \kappa$ given by equation Eq 20. The eigenvalues of $\rho$ are $\exp \left( \pm \frac{\mathbf{i} \pi}{4}\right), \exp \left( \pm \frac{3 \mathbf{i} \pi}{4}\right)\left(\right.$ where $\left.\mathbf{i}^{2}=-1\right)$. And we have the following decomposition: 


$$
\rho_{P}=P^{-1} \rho P=\left[\begin{array}{cccc}
\exp \left(\frac{\mathbf{i} \pi}{4}\right) & 0 & 0 & 0 \\
0 & \exp \left(-\frac{\mathbf{i} \pi}{4}\right) & 0 & 0 \\
0 & 0 & \exp \left(\frac{3 \mathbf{i} \pi}{4}\right) & 0 \\
0 & 0 & 0 & \exp \left(-\frac{3 \mathbf{i} \pi}{4}\right)
\end{array}\right] \text { with } P=\left[\begin{array}{cccc}
\mathbf{i} & -\mathbf{i} & \mathbf{i} & -\mathbf{i} \\
-1 & -1 & 1 & 1 \\
-\mathbf{i} & \mathbf{i} & \mathbf{i} & -\mathbf{i} \\
1 & 1 & 1 & 1
\end{array}\right]
$$

Then we can express in this basis the other generators:

$$
\sigma_{P}=P^{-1} \sigma P=\frac{\sqrt{2}}{4}\left[\begin{array}{cccc}
1 & \mathbf{i} \sqrt{3} & 1 & -\mathbf{i} \sqrt{3} \\
-\mathbf{i} \sqrt{3} & 1 & \mathbf{i} \sqrt{3} & 1 \\
1 & -\mathbf{i} \sqrt{3} & -1 & -\mathbf{i} \sqrt{3} \\
\mathbf{i} \sqrt{3} & 1 & \mathbf{i} \sqrt{3} & -1
\end{array}\right] \text { and } \kappa_{P}=P^{-1} \kappa P=\left[\begin{array}{cccc}
0 & \mathbf{i} & 0 & 0 \\
-\mathbf{i} & 0 & 0 & 0 \\
0 & 0 & 0 & \mathbf{i} \\
0 & 0 & -\mathbf{i} & 0
\end{array}\right]
$$

We denote $\mathbf{z}=\left(z_{1}, \bar{z}_{1}, z_{2}, \bar{z}_{2}\right)$ the complex coordinates associated to the eigenvectors of $\rho$ i.e the columns of $P$. Write $E$ in components as $\left(f_{1}, \bar{f}_{1}, f_{2}, \bar{f}_{2}\right)^{\mathbf{T}}$. We begin by describing the action of $\rho_{P}$ on the equivariant map $E$.

For all $\mathbf{z}$, the action is given by $\rho_{P} \cdot \mathbf{z}=\left(e^{\frac{\mathbf{i} \pi}{4}} z_{1}, e^{-\frac{\mathbf{i} \pi}{4}} \bar{z}_{1}, e^{\frac{3 \mathbf{i} \pi}{4}} z_{2}, e^{-\frac{3 \mathbf{i} \pi}{4}} \bar{z}_{2}\right)$ and the equivariance yields

$$
\left\{\begin{array}{c}
e^{\frac{\mathbf{i} \pi}{4}} f_{1}\left(z_{1}, \bar{z}_{1}, z_{2}, \bar{z}_{2}\right)=f_{1}\left(e^{\frac{\mathbf{i} \pi}{4}} z_{1}, e^{-\frac{\mathbf{i} \pi}{4}} \bar{z}_{1}, e^{\frac{3 \mathbf{i} \pi}{4}} z_{2}, e^{-\frac{3 \mathbf{i} \pi}{4}} \bar{z}_{2}\right) \\
e^{\frac{\mathbf{i} 3 \pi}{4}} f_{2}\left(z_{1}, \bar{z}_{1}, z_{2}, \bar{z}_{2}\right)=f_{2}\left(e^{\frac{\mathbf{i} \pi}{4}} z_{1}, e^{-\frac{\mathbf{i} \pi}{4}} \bar{z}_{1}, e^{\frac{3 \mathbf{i} \pi}{4}} z_{2}, e^{-\frac{3 \mathbf{i} \pi}{4}} \bar{z}_{2}\right)
\end{array}\right.
$$

We are looking for cubic equivariants of the form $\alpha z_{1}^{k_{1}} \bar{z}_{1}^{l_{1}} z_{2}^{k_{2}} \bar{z}_{2}^{l_{2}}$ satisfying the relation $k_{1}+k_{2}+l_{1}+l_{2}=3$. So with the first equation of (23) we simply get

$$
\alpha e^{\frac{\mathbf{i} \pi}{4}} z_{1}^{k_{1}} \bar{z}_{1}^{l_{1}} z_{2}^{k_{2}} \bar{z}_{2}^{l_{2}}=\alpha e^{\mathbf{i} \frac{\pi}{4}\left[\left(k_{1}-l_{1}\right)+3\left(k_{2}-l_{2}\right)\right]} z_{1}^{k_{1}} \bar{z}_{1}^{l_{1}} z_{2}^{k_{2}} \bar{z}_{2}^{l_{2}}
$$

In order that this is equivariant under the action of $\rho_{P}$ we have to impose:

$$
\left(k_{1}-l_{1}-1\right)+3\left(k_{2}-l_{2}\right)=8 n \text { with } n \in \mathbb{Z}
$$

which gives 5 elements in $f_{1}$.

$$
f_{1}\left(z_{1}, \bar{z}_{1}, z_{2}, \bar{z}_{2}\right)=a_{1} z_{1}^{2} \bar{z}_{1}+a_{2} z_{1} z_{2} \bar{z}_{2}+a_{3} z_{2}^{3}+a_{4} \bar{z}_{1}^{2} z_{2}+a_{5} \bar{z}_{1} \bar{z}_{2}^{2}
$$


with $\left(a_{i}\right)_{i=1 \ldots 5} \in \mathbb{C}^{5}$. In the same fashion the second equation of (23) gives 5 elements in $f_{2}$.

$$
f_{2}\left(z_{1}, \bar{z}_{1}, z_{2}, \bar{z}_{2}\right)=b_{1} z_{2}^{2} \bar{z}_{2}+b_{2} z_{1} \bar{z}_{1} z_{2}+b_{3} z_{1}^{3}+b_{4} z_{1} \bar{z}_{2}^{2}+b_{5} \bar{z}_{1}^{2} \bar{z}_{2}
$$

with $\left(b_{i}\right)_{i=1 \ldots . .5} \in \mathbb{C}^{5}$.

The action of $\kappa_{P}$ on $\mathbf{z}$ is given by $\kappa_{P} \cdot \mathbf{z}=\left(\mathbf{i} \bar{z}_{1},-\mathbf{i} z_{1}, \mathbf{i} \bar{z}_{2},-\mathbf{i} z_{2}\right)$. It is straightforward to see that this action imposes that $a_{1}, a_{2}, a_{5}, b_{1}, b_{2}, b_{5}$ are real and that $a_{3}, a_{4}, b_{3}, b_{4}$ are imaginary numbers. Then we can rewrite $f_{1}$ and $f_{2}$ as:

$$
\begin{aligned}
& f_{1}\left(z_{1}, \bar{z}_{1}, z_{2}, \bar{z}_{2}\right)=\alpha_{1} z_{1}^{2} \bar{z}_{1}+\alpha_{2} z_{1} z_{2} \bar{z}_{2}+\mathbf{i} \alpha_{3} z_{2}^{3}+\mathbf{i} \alpha_{4} \bar{z}_{1}^{2} z_{2}+\alpha_{5} \bar{z}_{1} \bar{z}_{2}^{2} \\
& f_{2}\left(z_{1}, \bar{z}_{1}, z_{2}, \bar{z}_{2}\right)=\beta_{1} z_{2}^{2} \bar{z}_{2}+\beta_{2} z_{1} \bar{z}_{1} z_{2}+\mathbf{i} \beta_{3} z_{1}^{3}+\mathbf{i} \beta_{4} z_{1} \bar{z}_{2}^{2}+\beta_{5} \bar{z}_{1}^{2} \bar{z}_{2}
\end{aligned}
$$

with $\left(\alpha_{i}, \beta_{i}\right)_{i=1 \ldots 5} \in(\mathbb{R} \times \mathbb{R})^{5}$.

\section{Action of $\sigma_{P}$ :}

The action of $\sigma_{P}$ on $\mathbf{z}$ is given by

$$
\sigma_{P} \cdot \mathbf{z}=\left(\begin{array}{c}
\frac{\sqrt{2}}{4}\left(z_{1}+z_{2}+\mathbf{i} \sqrt{3}\left(\bar{z}_{1}-\bar{z}_{2}\right)\right) \\
\frac{\sqrt{2}}{4}\left(\bar{z}_{1}+\bar{z}_{2}-\mathbf{i} \sqrt{3}\left(z_{1}-z_{2}\right)\right) \\
\frac{\sqrt{2}}{4}\left(z_{1}-z_{2}-\mathbf{i} \sqrt{3}\left(\bar{z}_{1}+\bar{z}_{2}\right)\right) \\
\frac{\sqrt{2}}{4}\left(\bar{z}_{1}-\bar{z}_{2}+\mathbf{i} \sqrt{3}\left(z_{1}+z_{2}\right)\right)
\end{array}\right)^{\mathbf{T}}
$$

and we find:

$$
\begin{aligned}
& f_{1}\left(z_{1}, \bar{z}_{1}, z_{2}, \bar{z}_{2}\right)=a z_{1}\left(\left|z_{1}\right|^{2}+\left|z_{2}\right|^{2}\right)+b\left(3 \sqrt{3} z_{1}\left|z_{1}\right|^{2}-\mathbf{i} z_{2}^{3}-3 \mathbf{i} \bar{z}_{1}^{2} z_{2}+\sqrt{3} \bar{z}_{1} \bar{z}_{2}^{2}\right) \\
& f_{2}\left(z_{1}, \bar{z}_{1}, z_{2}, \bar{z}_{2}\right)=a z_{2}\left(\left|z_{1}\right|^{2}+\left|z_{2}\right|^{2}\right)+b\left(3 \sqrt{3} z_{2}\left|z_{2}\right|^{2}+\mathbf{i} z_{1}^{3}+3 \mathbf{i} z_{1} \bar{z}_{2}^{2}+\sqrt{3} \bar{z}_{1}^{2} \bar{z}_{2}\right)
\end{aligned}
$$

with $(a, b) \in \mathbb{R}^{2}$. 


\section{C.2 Computational part of the proof of theorem 4}

Proposition 5. For the irreductible representation $\chi_{11}$, the four quintic equivariant maps are:

$$
\begin{gathered}
E_{5,1}(\mathbf{z})=\mathbf{z}|| \mathbf{z} \|^{4}, \quad E_{5,2}=\left[\begin{array}{c}
z_{1}^{4} \bar{z}_{2}+4 z_{2}^{3}\left|z_{1}\right|^{2}-z_{2}^{3}\left|z_{2}\right|^{2} \\
\bar{z}_{1}^{4} z_{2}+4 \bar{z}_{2}^{3}\left|z_{1}\right|^{2}-\bar{z}_{2}^{3}\left|z_{2}\right|^{2} \\
-\bar{z}_{1} z_{2}^{4}-4 z_{1}^{3}\left|z_{2}\right|^{2}+z_{1}^{3}\left|z_{1}\right|^{2} \\
-z_{1} \bar{z}_{2}^{4}-4 \bar{z}_{1}^{3}\left|z_{2}\right|^{2}+\bar{z}_{1}^{3}\left|z_{1}\right|^{2}
\end{array}\right] \\
E_{5,3}=\left[\begin{array}{c}
3 \bar{z}_{1}^{2} z_{2}\left|z_{2}\right|^{2}-z_{1}^{2} \bar{z}_{2}^{3}-2 \bar{z}_{1}^{2}\left|z_{1}\right|^{2} z_{2} \\
3 z_{1}^{2} \bar{z}_{2}\left|z_{2}\right|^{2}-\bar{z}_{1}^{2} z_{2}^{3}-2 z_{1}^{2}\left|z_{1}\right|^{2} \bar{z}_{2} \\
-3 z_{1} \bar{z}_{2}^{2}\left|z_{1}\right|^{2}+\bar{z}_{1}^{3} z_{2}^{2}+2 z_{1} \bar{z}_{2}^{2}\left|z_{2}\right|^{2} \\
-3 \bar{z}_{1} z_{2}^{2}\left|z_{1}\right|^{2}+z_{1}^{3} \bar{z}_{2}^{2}+2 \bar{z}_{1} z_{2}^{2}\left|z_{2}\right|^{2}
\end{array}\right], \quad E_{5,4}=\left[\begin{array}{c}
-5 \bar{z}_{1}^{4} \bar{z}_{2}+\bar{z}_{2}^{5} \\
-5 z_{1}^{4} z_{2}+z_{2}^{5} \\
5 \bar{z}_{1} \bar{z}_{2}^{4}-\bar{z}_{1}^{5} \\
5 z_{1} z_{2}^{4}-z_{1}^{5}
\end{array}\right]
\end{gathered}
$$

Proof. Let $E$ denote a homageneous cubic equivariant mapping. Thanks to proposition 3 of appendix A the generators of $\mathcal{G}$ are given in matrix form in equation (21). The eigenvalues of $\rho$ are still $\exp \left( \pm \frac{\mathbf{i} \pi}{4}\right), \exp \left( \pm \frac{3 \mathbf{i} \pi}{4}\right)$. And we have the following decomposition:

$$
\rho_{P}=P^{-1} \rho P=\left[\begin{array}{cccc}
\exp \left(\frac{\mathbf{i} \pi}{4}\right) & 0 & 0 & 0 \\
0 & \exp \left(-\frac{\mathbf{i} \pi}{4}\right) & 0 & 0 \\
0 & 0 & \exp \left(\frac{3 \mathbf{i} \pi}{4}\right) & 0 \\
0 & 0 & 0 & \exp \left(-\frac{3 \mathbf{i} \pi}{4}\right)
\end{array}\right] \text { with } P=\left[\begin{array}{cccc}
\mathbf{i} & -\mathbf{i} & -\mathbf{i} & \mathbf{i} \\
-1 & -1 & 1 & 1 \\
\mathbf{i} & -\mathbf{i} & \mathbf{i} & -\mathbf{i} \\
1 & 1 & 1 & 1
\end{array}\right]
$$

Then we can express in this basis the other generators:

$$
\sigma_{P}=P^{-1} \sigma P=\frac{\sqrt{2}}{2}\left[\begin{array}{cccc}
-1 & 0 & -\mathbf{i} & 0 \\
0 & -1 & 0 & \mathbf{i} \\
\mathbf{i} & 0 & 1 & 0 \\
0 & -\mathbf{i} & 0 & 1
\end{array}\right] \text { and } \kappa_{P}=P^{-1} \kappa P=\left[\begin{array}{cccc}
0 & \mathbf{i} & 0 & 0 \\
-\mathbf{i} & 0 & 0 & 0 \\
0 & 0 & 0 & -\mathbf{i} \\
0 & 0 & \mathbf{i} & 0
\end{array}\right]
$$

We denote $\mathbf{z}=\left(z_{1}, \bar{z}_{1}, z_{2}, \bar{z}_{2}\right)$ the complex coordinates associated to the eigenvectors of $\rho$ i.e the columns of $P$. Write $E$ in components as $\left(f_{1}, \bar{f}_{1}, f_{2}, \bar{f}_{2}\right)^{\mathbf{T}}$. The action of $\rho_{P}$ on a quintic equivariant map of the form $\alpha z_{1}^{k_{1}} \bar{z}_{1}^{l_{1}} z_{2}^{k_{2}} \bar{z}_{2}^{l_{2}}$ with the relation $k_{1}+k_{2}+l_{1}+l_{2}=5$ implies that $\left(k_{1}-l_{1}-1\right)+3\left(k_{2}-l_{2}\right)=8 n$ with $n \in \mathbb{Z}$, which gives 14 elements in $f_{1}$.

$$
f_{1}\left(z_{1}, \bar{z}_{1}, z_{2}, \bar{z}_{2}\right)=a_{1} z_{1}^{3} \bar{z}_{1}^{2}+a_{2} z_{1} z_{2}^{2} \bar{z}_{2}^{2}+a_{3} z_{1}^{2} \bar{z}_{1} z_{2} \bar{z}_{2}+a_{4} z_{1}^{4} \bar{z}_{2}+a_{5} \bar{z}_{1}^{2} z_{2}^{2} \bar{z}_{2}+a_{6} z_{1} \bar{z}_{1} z_{2}^{3}+a_{7} z_{1}^{3} z_{2}^{2}
$$




$$
+a_{8} z_{2}^{4} \bar{z}_{2}+a_{9} z_{1}^{2} \bar{z}_{2}^{3}+a_{10} z_{1} \bar{z}_{1}^{2} \bar{z}_{2}^{2}+a_{11} \bar{z}_{1} z_{2} \bar{z}_{2}^{3}+a_{12} \bar{z}_{1}^{4} \bar{z}_{2}+a_{13} \bar{z}_{2}^{5}+a_{14} z_{1} \bar{z}_{1}^{3} z_{2}
$$

And we also obtain 14 elements in $f_{2}$ with the same method:

$$
\begin{gathered}
f_{2}\left(z_{1}, \bar{z}_{1}, z_{2}, \bar{z}_{2}\right)=b_{1} z_{1}^{2} \bar{z}_{1}^{2} z_{2}+b_{2} z_{1} \bar{z}_{1} z_{2}^{2} \bar{z}_{2}+b_{3} z_{2}^{3} \bar{z}_{2}^{2}+b_{4} z_{1}^{4} \bar{z}_{1}+b_{5} z_{1}^{3} z_{2} \bar{z}_{2}+b_{6} \bar{z}_{1}^{3} z_{2}^{2}+b_{7} z_{1}^{2} z_{2}^{3} \\
+b_{8} \bar{z}_{1} z_{2}^{4}+b_{9} z_{1} z_{2} \bar{z}_{2}^{3}+b_{10} z_{1}^{2} \bar{z}_{1} \bar{z}_{2}^{2}+b_{11} z_{1} \bar{z}_{1}^{3} \bar{z}_{2}+b_{12} \bar{z}_{1}^{2} z_{2} \bar{z}_{2}^{2}+b_{13} \bar{z}_{1}^{5}+b_{14} \bar{z}_{1} \bar{z}_{2}^{4}
\end{gathered}
$$

where $\left(a_{j}\right)_{j=1 \ldots 14} \in \mathbb{C}^{14}$ and $\left(b_{j}\right)_{j=1 \ldots 14} \in \mathbb{C}^{14}$.

The action of $\kappa_{P}$ implies that the coefficients $\left(a_{j}, b_{j}\right)_{j=1 \ldots 14}$ are real. The action of $\sigma_{P}$ is $\sigma_{P} \cdot \mathbf{z}=$ $\frac{\sqrt{2}}{2}\left(-z_{1}-\mathbf{i} z_{2},-\bar{z}_{1}+\mathbf{i} \bar{z}_{2}, \mathbf{i} z_{1}+z_{2},-\mathbf{i} \bar{z}_{1}+\bar{z}_{2}\right)$ and we obtain:

$$
\begin{gathered}
f_{1}\left(z_{1}, \bar{z}_{1}, z_{2}, \bar{z}_{2}\right)=a\left(z_{1}\left|z_{1}\right|^{4}+z_{1}\left|z_{2}\right|^{4}+2 z_{1}\left|z_{1}\right|^{2}\left|z_{2}\right|^{2}\right)+b\left(z_{1}^{4} \bar{z}_{2}+4 z_{2}^{3}\left|z_{1}\right|^{2}-z_{2}^{3}\left|z_{2}\right|^{2}\right) \\
+c\left(3 \bar{z}_{1}^{2} z_{2}\left|z_{2}\right|^{2}-z_{1}^{2} \bar{z}_{2}^{3}-2 \bar{z}_{1}^{2}\left|z_{1}\right|^{2} z_{2}\right)+d\left(-5 \bar{z}_{1}^{4} \bar{z}_{2}+\bar{z}_{2}^{5}\right) \\
f_{2}\left(z_{1}, \bar{z}_{1}, z_{2}, \bar{z}_{2}\right)=a\left(z_{2}\left|z_{2}\right|^{4}+z_{2}\left|z_{1}\right|^{4}+2 z_{2}\left|z_{1}\right|^{2}\left|z_{2}\right|^{2}\right)+b\left(-\bar{z}_{1} z_{2}^{4}-4 z_{1}^{3}\left|z_{2}\right|^{2}+z_{1}^{3}\left|z_{1}\right|^{2}\right) \\
+c\left(-3 z_{1} \bar{z}_{2}^{2}\left|z_{1}\right|^{2}+\bar{z}_{1}^{3} z_{2}^{2}+2 z_{1} \bar{z}_{2}^{2}\left|z_{2}\right|^{2}\right)+d\left(5 \bar{z}_{1} \bar{z}_{2}^{4}-\bar{z}_{1}^{5}\right)
\end{gathered}
$$

with $(a, b, c, d) \in \mathbb{R}^{4}$. Thus, we find 4 equivariant maps which is in agreement with computation of the Molien serie of appendix B.

\section{Fixed-point subspaces}

\section{D.1 Proof of Lemma 7}

To complete the proof of Lemma 7 we give the matrix of $\sigma, \tilde{\sigma}, \epsilon, \kappa, \kappa^{\prime}$ and $\kappa^{\prime \prime}$ in the basis associated to coordinates $\left(z_{1}, \bar{z}_{1}, z_{2}, \bar{z}_{2}\right)$.

$$
\sigma=\frac{\sqrt{2}}{4}\left[\begin{array}{cccc}
1 & \mathbf{i} \sqrt{3} & 1 & -\mathbf{i} \sqrt{3} \\
-\mathbf{i} \sqrt{3} & 1 & \mathbf{i} \sqrt{3} & 1 \\
1 & -\mathbf{i} \sqrt{3} & -1 & -\mathbf{i} \sqrt{3} \\
\mathbf{i} \sqrt{3} & 1 & \mathbf{i} \sqrt{3} & -1
\end{array}\right], \quad \tilde{\sigma}=\frac{\sqrt{2}}{4}\left[\begin{array}{cccc}
1 & -\mathbf{i} \sqrt{3} & -1 & -\mathbf{i} \sqrt{3} \\
\mathbf{i} \sqrt{3} & 1 & \mathbf{i} \sqrt{3} & -1 \\
-1 & -\mathbf{i} \sqrt{3} & -1 & \mathbf{i} \sqrt{3} \\
\mathbf{i} \sqrt{3} & -1 & -\mathbf{i} \sqrt{3} & -1
\end{array}\right]
$$




$$
\begin{gathered}
\epsilon=\frac{1}{4}\left[\begin{array}{cccc}
1-\mathbf{i} & \sqrt{3}(\mathbf{i}-1) & -1-\mathbf{i} & \sqrt{3}(1+\mathbf{i}) \\
-\sqrt{3}(1+\mathbf{i}) & 1+\mathbf{i} & \sqrt{3}(1-\mathbf{i}) & \mathbf{i}-1 \\
1-\mathbf{i} & \sqrt{3}(1-\mathbf{i}) & 1+\mathbf{i} & \sqrt{3}(1+\mathbf{i}) \\
\sqrt{3}(1+\mathbf{i}) & 1+\mathbf{i} & \sqrt{3}(1-\mathbf{i}) & 1-\mathbf{i}
\end{array}\right], \quad \kappa=\left[\begin{array}{cccc}
0 & \mathbf{i} & 0 & 0 \\
-\mathbf{i} & 0 & 0 & 0 \\
0 & 0 & 0 & \mathbf{i} \\
0 & 0 & -\mathbf{i} & 0
\end{array}\right] \\
\kappa^{\prime}=\frac{\sqrt{2}}{2}\left[\begin{array}{cccc}
0 & \mathbf{i}-1 & 0 & 0 \\
-1-\mathbf{i} & 0 & 0 & 0 \\
0 & 0 & 0 & -1-\mathbf{i} \\
0 & 0 & -1+\mathbf{i} & 0
\end{array}\right], \quad \kappa^{\prime \prime}=\frac{\sqrt{2}}{4}\left[\begin{array}{cccc}
\sqrt{3} & \mathbf{i} & -\sqrt{3} & \mathbf{i} \\
-\mathbf{i} & \sqrt{3} & -\mathbf{i} & -\sqrt{3} \\
-\sqrt{3} & \mathbf{i} & -\sqrt{3} & -\mathbf{i} \\
-\mathbf{i} & -\sqrt{3} & \mathbf{i} & -\sqrt{3}
\end{array}\right]
\end{gathered}
$$

\section{D.2 Proof of Lemma 10}

To complete the proof of Lemma 10 we give the matrix of $\sigma, \kappa, \kappa^{\prime}$ and $\kappa^{\prime \prime}$ in the basis associated to coordinates $\left(z_{1}, \bar{z}_{1}, z_{2}, \bar{z}_{2}\right)$.

$$
\begin{gathered}
\sigma=\frac{\sqrt{2}}{2}\left[\begin{array}{cccc}
-1 & 0 & -\mathbf{i} & 0 \\
0 & -1 & 0 & \mathbf{i} \\
\mathbf{i} & 0 & 1 & 0 \\
0 & -\mathbf{i} & 0 & 1
\end{array}\right], \quad \kappa=\left[\begin{array}{cccc}
0 & \mathbf{i} & 0 & 0 \\
-\mathbf{i} & 0 & 0 & 0 \\
0 & 0 & 0 & -\mathbf{i} \\
0 & 0 & \mathbf{i} & 0
\end{array}\right] \\
\kappa^{\prime}=\frac{\sqrt{2}}{2}\left[\begin{array}{cccc}
0 & \mathbf{i}-1 & 0 & 0 \\
-1-\mathbf{i} & 0 & 0 & 0 \\
0 & 0 & 0 & 1+\mathbf{i} \\
0 & 0 & 1-\mathbf{i} & 0
\end{array}\right], \quad \kappa^{\prime \prime}=\frac{\sqrt{2}}{2}\left[\begin{array}{cccc}
0 & -\mathbf{i} & 0 & -1 \\
\mathbf{i} & 0 & -1 & 0 \\
0 & -1 & 0 & -\mathbf{i} \\
-1 & 0 & \mathbf{i} & 0
\end{array}\right]
\end{gathered}
$$

\section{References}

[1] M.A.D. Aguiar, S.B.S.D. Castro, and I.S. Labouriau. Dynamics near a heteroclinic network. Nonlinearity, 18, 2005.

[2] D. Armbruster, J. Guckenheimer and P. Holmes Heteroclinic cycles and modulated waves in systems with $\mathrm{O}(2)$ symmetry. Phyisica D, 29, 257-282, 1988.

[3] P. Ashwin and M. Field. Heteroclinic networks in coupled cell systems. Archive for Rational Mechanics and Analysis, 148(2):107-143, 1999.

[4] R. Aurich and F. Steiner. Periodic-orbit sum rules for the hadamard-gutzwiller model. Physica D, 39:169-193, 1989. 
[5] N.L. Balazs and A. Voros. Chaos on the pseudosphere. Physics Reports, 143(3):109-240, 1986.

[6] J. Bigun and G. Granlund. Optimal orientation detection of linear symmetry. In Proc. First Int'l Conf. Comput. Vision, pages 433-438. EEE Computer Society Press, 1987.

[7] P. C. Bressloff and J. D. Cowan. A spherical model for orientation and spatial frequency tuning in a cortical hypercolumn. Philosophical Transactions of the Royal Society B, 2003.

[8] P.C. Bressloff and J.D. Cowan. SO(3) symmetry breaking mechanism for orientation and spatial frequency tuning in the visual cortex. Phys. Rev. Lett., 88(7), feb 2002.

[9] P.C. Bressloff and J.D. Cowan. The visual cortex as a crystal. Physica D: Nonlinear Phenomena, 173(3-4):226-258, dec 2002.

[10] P.C. Bressloff, J.D. Cowan, M. Golubitsky, P.J. Thomas, and M.C. Wiener. Geometric visual hallucinations, Euclidean symmetry and the functional architecture of striate cortex. Phil. Trans. R. Soc. Lond. B, 306(1407):299-330, mar 2001.

[11] P. Chossat and R. Lauterbach. Methods in Equivariant Bifurcations and Dynamical Systems. World Scientific Publishing Company, 2000.

[12] P. Chossat, R. Lauterbach, and I. Melbourne. Steady-state bifurcation with 0 (3)-symmetry. Archive for Rational Mechanics and Analysis, 113(4):313-376, 1990.

[13] P. Chossat and O. Faugeras. Hyperbolic planforms in relation to visual edges and textures perception. Plos Comput Biol, 5(12):e1000625, December 2009.

[14] P. Chossat, G. Faye, and O. Faugeras. Bifurcation of hyperbolic planforms. Journal of Nonlinear Science, February 2011.

[15] P.G. Ciarlet and J.L. Lions, editors. Handbook of Numerical Analysis. Volume II. Finite Element Methods (part1). North-Holland, 1991.

[16] J.H Conway and D.A Smith. On Quaternions and Octonions, Their Geometry, Arithmetic, and Symmetry. AK Peters, 2003.

[17] A. Fässler and E.L. Stiefel. Group theoretical methods and their applications. Birkhäuser, 1992.

[18] G. Faye, P. Chossat, and O. Faugeras. Analysis of a hyperbolic geometric model for visual texture perception. The Journal of Mathematical Neuroscience, 1(4), 2011.

[19] M. Field. Equivariant bifurcation theory and symmetry breaking. Journal of Dynamics and Differential Equations, 1(4):369-421, 1989. 
[20] M. Field. Lectures on bifurcations, dynamics and symmetry. Pitman Research Notes in Mathematics Series, 1996.

[21] M. Field, J. Swift. Stationary bifurcation to limit cycles and heteroclinic cycles Nonlinearity, 4: 1001-1043, 1991.

[22] J.A. Goldberg, U. Rokni and H. Sompolinsky. Patterns of ongoing activity and the functional architecture of the primary visual cortex. Neuron, vol 42, pp 489-500, 2004.

[23] M. Golubitsky, I. Stewart, and D.G. Schaeffer. Singularities and Groups in Bifurcation Theory, volume II. Springer, 1988.

[24] J. Guckenheimer and P. Holmes Structurally stable heteroclinic cycles. Math. Proc. Cambridge Phil. Soc. 103, 189-192, 1988.

[25] V. Guillemin and A. Pollack. Differential topology. Chelsea Pub Co, 2010.

[26] M. Haragus and G. Iooss. Local bifurcations, center manifolds, and normal forms in infinite dimensional systems. EDP Sci. Springer Verlag UTX series, 2010.

[27] S. Helgason. Groups and geometric analysis, volume 83 of Mathematical Surveys and Monographs. American Mathematical Society, 2000.

[28] R.B. Hoyle. Pattern formation: an introduction to methods. Cambridge Univ Pr, 2006.

[29] S. Katok. Fuchsian Groups. Chicago Lectures in Mathematics. The University of Chicago Press, 1992.

[30] T. Kenet, D. Bibitchkov, M. Tsodyks, A. Grinvald and A. Arieli. Spontaneously emerging cortical representations of visual attributes. Nature, vol 425, issue 6961, pp 954-956, 2003.

[31] V. Kirk and M. Silber. A competition between heteroclinic cycles. Nonlinearity, 7:1605, 1994.

[32] H. Knutsson. Representing local structure using tensors. In Scandinavian Conference on Image Analysis, pages 244-251, 1989.

[33] M. Krupa and I. Melbourne. Nonasymptotically stable attractors in o(2) mode interactions. Normal forms and homoclinic chaos (Waterloo, ON, 1992), 4:219-232, 1992.

[34] M. Krupa and I. Melbourne. Asymptotic stability of heteroclinic cycles in systems with symmetry. Ergodic Theory and Dynamical Systems, 15(01):121-147, 1995. 
[35] M. Krupa and I. Melbourne. Asymptotic stability of heteroclinic cycles in systems with symmetry. ii. Proceedings of the Royal Society of Edinburgh: Section A Mathematics, 134(06):1177-1197, 2004.

[36] R. Lauterbach and P. Matthews. Do absolutely irreducible group actions have odd dimensional fixed point spaces? ArXiv, (1011.3986), 2010.

[37] W. Miller Symmetry groups and their applications. Academic press, 50, 1972.

[38] M.I. Rabinovich, R. Huerta, P. Varona and V.S. Afraimovich. Transient cognitive dynamics, metastability, and decision making PLoS Comput. Biol, e1000072, 2008.

[39] M.I. Rabinovich, M.K. Muezzinoglu, I.Strigo and A. Bystritsky. Dynamical Principles of EmotionCognition Interaction: Mathematical Images of Mental Disorders . PloS One, e12547, 2010.

[40] D.L. Ringach. Neuroscience: states of mind. Nature, vol 425, 2003.

[41] C. Schmit. Quantum and classical properties of some billiards on the hyperbolic plane. Chaos and Quantum Physics, pages 335-369, 1991.

[42] R. Veltz and O. Faugeras. Local/global analysis of the stationary solutions of some neural field equations. SIAM Journal on Applied Dynamical Systems, 2010.

[43] H.R. Wilson and J.D. Cowan. Excitatory and inhibitory interactions in localized populations of model neurons. Biophys. J., 12:1-24, 1972.

[44] H.R. Wilson and J.D. Cowan. A mathematical theory of the functional dynamics of cortical and thalamic nervous tissue. Biological Cybernetics, 13(2):55-80, September 1973. 